\begin{tabular}{|c|c|c|c|c|c|}
\hline \multicolumn{6}{|c|}{ DISTRIBUTION SHEET } \\
\hline \multirow{2}{*}{$\begin{array}{l}\text { To } \\
\text { Distribution }\end{array}$} & \multirow{2}{*}{\multicolumn{3}{|c|}{$\begin{array}{l}\text { From } \\
\text { Characterization Plans and Reports }\end{array}$}} & Page 1 of & \\
\hline & & & & Date: & $05 / 16 / 95$ \\
\hline \multirow{2}{*}{\multicolumn{4}{|c|}{$\begin{array}{l}\text { Project Title/Work Order } \\
\text { WHC-SD-WM-DP-112, Rev. 0, "45-Day Safety Screen Results and } \\
\text { Final Report for Tank 241-C-203, Auger Samples } 95-A U G-020 \text { and } \\
\text { 95-AUG-021" }\end{array}$}} & EDT NO.: & EDT-612156 \\
\hline & & & & ECN NO.: & $N / A$ \\
\hline & Name & MSIN & $\begin{array}{l}\text { Text With } \\
\text { all Attach }\end{array}$ & $\begin{array}{l}\text { EDT/ECN } \\
\text { ONLY }\end{array}$ & \\
\hline
\end{tabular}

Pacific Northwest Laboratory

J. R. Gormsen

S. J. Harris

K. L. Silvers

U.S. Department of Energy, RL
$\mathrm{K} 7-28$

$\mathrm{K} 7-22$

P7-27

S7-54

G3-21

S7-30

S7-15

R2-12

H4-19

S7-31

H5- 49

H4-21

S6-31

T6-07

S7-15

R2-11

T6-06

H5- 49

S7-14

S7-15

S7-15

H4-25

R2-12

S7-15

R4-01

L8-04

H6-08

T6-03

L8-07

R2-12

R1-20 $x$

$\mathrm{X}$

$x$

$x$

$x$

$x$

$x$

$x$

$x$.

$x$

$x$

TCRC

TFIC (Tank Farm Information Center) 


\section{DISCLAIMER}

Portions of this document may be illegible in electronic image products. Images are produced from the best available original document. 


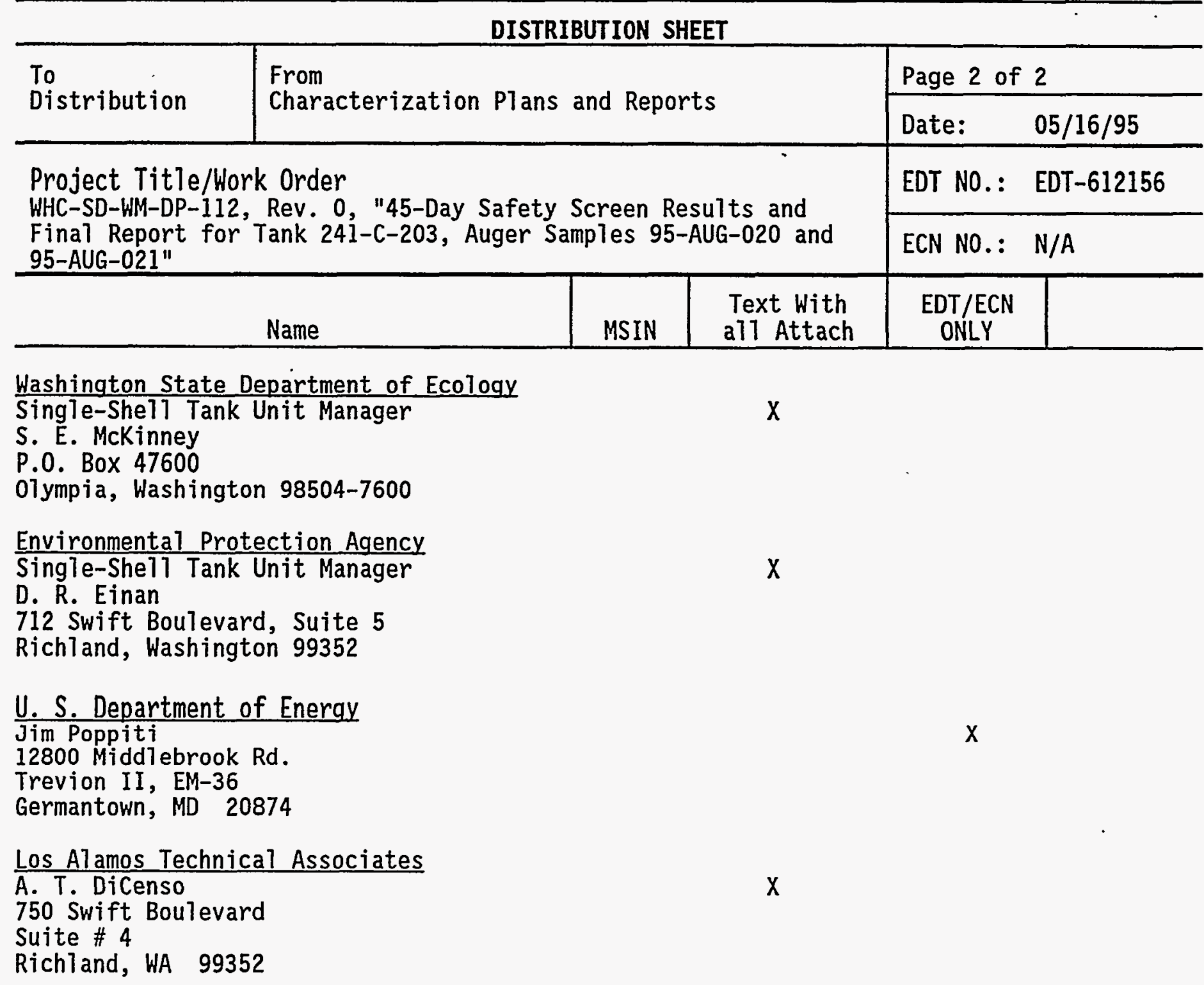




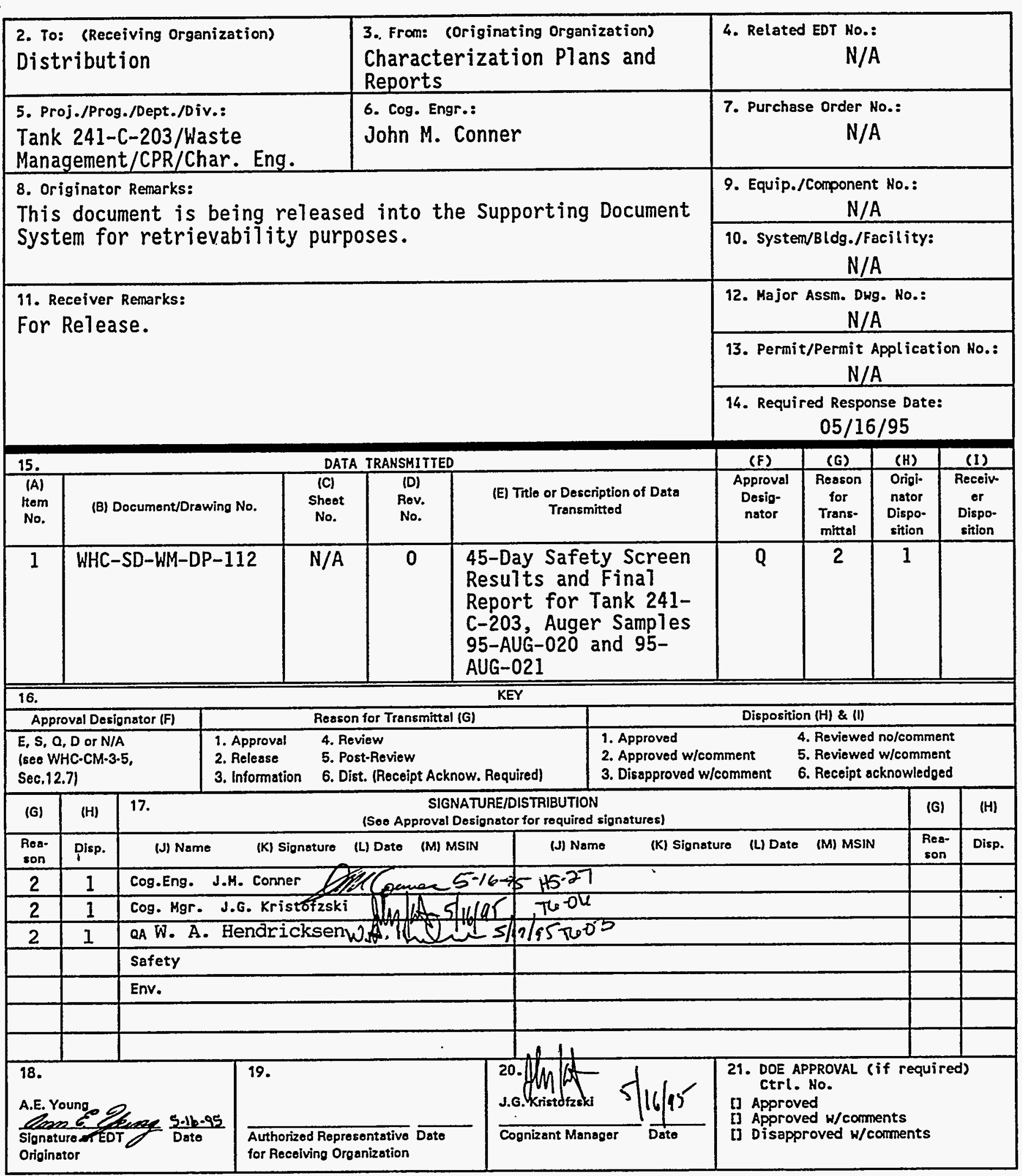




\section{RELEASE AUTHORIZATION}

\begin{tabular}{|ll|}
\hline Document Number: & WHC-SD-WM-DP-112, REV 0 \\
\hline Document Title: & $\begin{array}{l}\text { 45-Day Safety Screen Results and Final Report for } \\
\text { Tank 241-C-203, Auger Samples 95-AUG-020 and 95-AUG- } \\
021\end{array}$ \\
\hline Release Date: & $5 / 18 / 95$ \\
\hline
\end{tabular}

\section{This document was reviewed following the procedures described in WHC-CM-3-4 and is:}

\section{APPROVED FOR PUBLIC RELEASE}

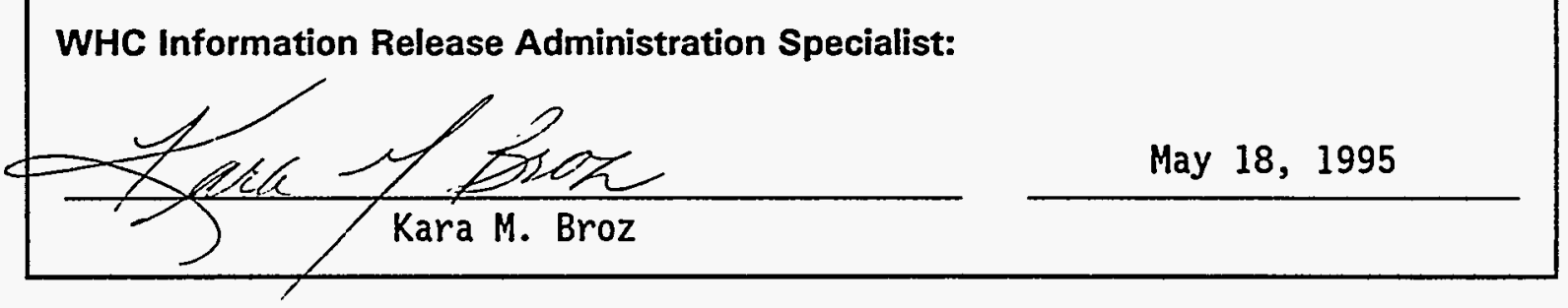

TRADEMARK DISCLAIMER. Reference herein to any specific commercial product, process, or service by trade name, trademark, manufacturer, or otherwise, does not necessarily constitute or imply its endorsement, recormendation, or favoring by the United States Government or any agency thereof or its contractors or subcontractors.

This report has been reproduced from the best available copy. Available in paper copy and microfiche. Printed in the United States of America. Available to the U.S. Department of Energy and its contractors from:

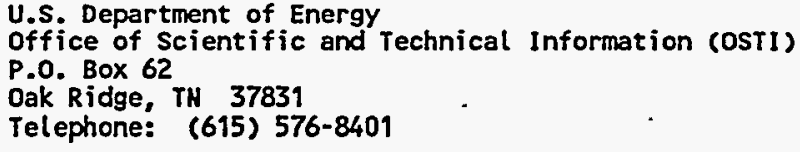

U.S. Department of Commerce National Technical Information Service (NTIS) 5285 Port Royal Road

Springfield, VA 22161

Telephone: (703) 487-4650 
2. Title

45-Day Safety Screen Results and Final Report for Tank 241-C-203, Auger Samples 95-AUG-020 and 95AUG-021

5. Key Hords

45-Day Safety Screen, Safety Screen, Final Report, Tank 241-C-203, Tank C-203, C-203, Auger Sample, 95-AUG-020, 95-AUG-021
3. Number

WHC-SD-HM-DP-112

4. Rev No.

0
6. Author

Name: John M. Conner

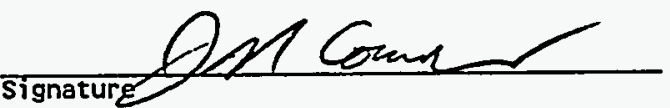

Organization/Charge Code

\section{Abstract}

$N / A$

\section{DISCLAIMER}

This report was prepared as an account of work sponsored by an agency of the United States Government. Neither the United States Government nor any agency thereof, nor any of their employees, makes any warranty, express or implied, or assumes any legal liability or responsibility for the accuracy, completeness, or usefulness of any information, apparatus, product, or process disclosed, or represents that its use would not infringe privately owned rights. Reference herein to any specific commercial product, process, or service by trade name, trademark, manufacturer, or otherwise does not necessarily constitute or imply its endorsement, recommendation, or favoring by the United States Government or any agency thereof. The views and opinions of authors expressed herein do not necessarily state or reflect those of the United States Government or any agency thereof.

8. RELEASE STAMP 
WHC-SD-WM-DP-112, REV. 0

\section{ANALYTICAL SERVICES}

45-DAY SAFETY SCREEN RESULTS AND FINAL REPORT FOR TANK 241-C-203, AUGER SAMPLES 95-AUG-020 AND 95-AUG-021

Date Printed: $\quad$ MAY 17, 1995 
WHC-SD-HM-DP-112, REV. 0

TABLE OF CONTENTS

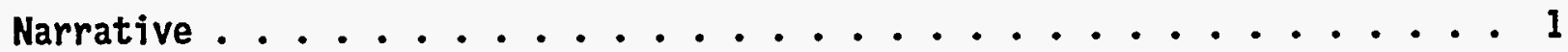

Sample Data Summary . . . . . . . . . . . . . . 6

Photographs ...................... 9

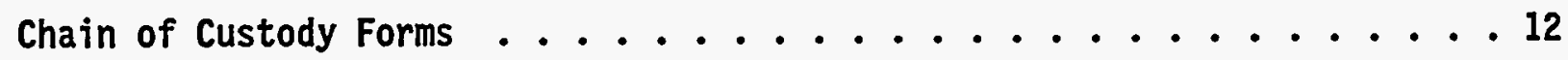

Sample Analyses Results . . . . . . . . . . . . 15

Extrusion Worklist \#1005 ............... . 16

Extrusion Worklist \# 1006 ................ . 17

Archive Workl ist \# $1276 \ldots . . . . . . . . .18$

Bulk Density Work1 ist \# 1322 . . . . . . . . . . . . 19

Fusion Worklist \# 1173 ................. 20

Fusion Worklist \# $1174 \ldots . . . . . . . . . .21$

Differential Scanning Calorimetry(DSC)

DSC Worklist \# 1216 ................... 22

DSC Worklist \# 1217 ................. 28

DSC Worklist \# $1401 \ldots \ldots 35$

Thermogravimetric Analyses (TGA)

TGA Worklist \# 1237 .................. 37

TGA Worklist \# $1238 \ldots . . . . . . . . . . . .43$

Total Alpha/Total Beta Worklist \#1318 . . . . . . . . . . 52

Total Alpha/Total Beta Workl ist \#1366 ............... . 60

Total Alpha/Total Beta Workl ist \# 1367 . . . . . . . . . . . . 68

Total Alpha/Total Beta Worklist \#1402 . : . . . . . . . . . . . 75

This Document consists of pages 1 through 83. 
WHC-SD-WM-DP-112, REV. 0

NARRATIVE 


\author{
WHC-SD-WM-DP-112, REV. 0 \\ 45-DAY SAFETY SCREEN RESULTS AND FINAL REPORT FOR TANK 241-C-203, \\ AUGER SAMPLES 95-AUG-020 AND 95-AUG-021
}

\title{
ANALYTICAL SUMMARY
}

Two core samples from tank 241-C-203 (C-203) were received at the 222-S Laboratories and underwent safety screening analysis, consisting of differential scanning calorimetry (DSC), thermogravimetric analysis (TGA), and total alpha activity. No notification limits were exceeded for the analyses. Two samples were submitted for a density determination at the request of Characterization Plant Engineering.

\section{$\underline{\text { SCOPE }}$}

This document serves as the 45-day report deliverable for the tank C-203 auger samples collected on Apri1 5, 1995 (samples 95-AUG-20 and 95-AUG-021). As no secondary analyses were required and no other analyses have been requested, this document also serves as the final report for $\mathrm{C}-203$ auger sampling. Each sample was received, extruded, and analyzed by the 222-S Laboratories in accordance with the Tank Characterization P1an (TCP) referenced below. Included in this report are the primary safety screening results (DSC, TGA, and alpha) and density results. The worklists and raw data are included in this report. Photographs of the auger samples were taken during extrusion and, although not included in this report, are available.

\section{SAMPLE RECEIPT, EXTRUSION, AND SUBSAMPLING}

\section{5-AUG-020}

Sample 95-AUG-020 was collected from riser 7 (east coordinate) of tank C-203 using a 20-inch auger sampler. The sample was taken on Apri1 5, 1995 at 0943 hours. It was received into the 222-S Laboratories on April 6 and extruded on April 7. The sampler was almost completely fuil. A total of 493.21 grams of solid material were collected, with no drainable liquid. The samples appeared to be yellow/cream colored overlaid with brown to dark brown sludge. Upon subsampling (and incidental mixing), the material appeared brown. The auger sample was divided into half segments for analyses. Archive samples of each half segment have been retained in the hot cell. The subsamples are identified in Table 1 .

\section{5-AUG-021}

Sample 95-AUG-021 was collected from riser 7 (west coordinate) of tank C-203 using a 20-inch auger sampler. The sample was taken on Apri1 5, 1995 at 1135 hours. It was received into the 222-S Laboratories on April 6 and extruded on April 7. The auger was completely full. A total of 627.69 grams of solid 
WHC-SD-WM-DP-112, REV. 0

material were collected, with no drainable liquid. The solids ranged from a yellow/cream color to dark brown. More yellow material was noticed on the upper half than on the lower half of the auger. Upon subsampling (and incidental mixing), the material appeared brown. The auger sample was divided into half segments for analyses. Archive samples of each half segment have been retained in the hot cell. The subsamples are identified in Table 1.

Table 1. Sample Identification

\begin{tabular}{|c|c|c|}
\hline LABCORE \# & Sample Description & Analysis \\
\hline S95T000699 & 95-AUG-020 (riser 7-east) auger sample & extrusion \\
\hline S95T000702 & 95-AUG-021 (riser 7-west) auger sample & extrusion \\
\hline S95T000798 & 95-AUG-021 upper half & density \\
\hline S95T000799 & 95-AUG-021 upper half, direct analysis & $\mathrm{DSC} / \mathrm{TGA}$ \\
\hline S95T000800 & 95-AUG-021 upper half, fusion/digest & Total alpha \\
\hline S95T000801 & 95-AUG-021 upper ha7f, archive & archive \\
\hline S95T000802 & 95-AUG-021 lower half & density \\
\hline $595 T 000803$ & 95-AUG-021 lower half, direct analysis & $\mathrm{DSC} / \mathrm{TGA}$ \\
\hline S95T000804 & 95-AUG-021 lower half, fusion/digest & Total alpha \\
\hline S95T000805 & 95-AUG-021 lower half, archive & archive \\
\hline S95T000806 & 95-AUG-020 lower half & $\mathrm{n} / \mathrm{a}$ \\
\hline S95T000807 & 95-AUG-020 lower half, direct analysis & DSC/TGA \\
\hline S95T000808 & 95-AUG-020 lower half, fusion/digest & Total alpha \\
\hline S95T000809 & 95-AUG-020 lower half, archive & archive \\
\hline $595 T 000810$ & 95-AUG-020 upper half & $n / a$ \\
\hline S95T000811 & 95-AUG-020 upper half, direct analysis & $\mathrm{DSC} / \mathrm{TGA}$ \\
\hline S95T000812 & 95-AUG-020 upper half, fusion/digest & Total alpha \\
\hline S95T000813 & 95-AUG-020 upper half, archive & archive \\
\hline
\end{tabular}

ANALYTICAL RESULTS

Analytical results are presented in Tables 2 and 3 , with the applicable notification limits shaded. 
WHC-SD-HM-DP-112, REV. 0

\section{DSC (Energetics Content)}

DSC analyses were performed under a nitrogen atmosphere using either procedure LA-514-113, Rev. B-1 (Mett7er instrument) or procedure LA-514-114, Rev. B-0 (Perkin-Elmer instrument). The results are given in Tables 2 and 3. Exotherms were detected for a 71 four samples and their duplicates. The exotherms ranged from 30.82 to $97.33 \mathrm{~J} / \mathrm{g}$ on a dry weight basis, or approximately 6 to 20 percent of the safety screening limit of $481 \mathrm{~J} / \mathrm{g}$. The relative percent differences (RPDs) between sample and duplicate results ranged from 1.04 to 6.38 on a dry weight basis. These results are within the TCP target of 10\%. The three Laboratory Measurement Control System (LMCS) control standards exhibited recoveries of between 94.27 and 99.82 percent, which were within the program's specified accuracy control limits of 90 to 110 percent recovery.

\section{TGA (Moisture Content)}

Height percent water was performed under a nitrogen atmosphere using either procedure LA-560-112, Rev. A-2 (Mettler instrument), or procedure LA-514-114, Rev. B-0 (Perkin-Elmer instrument). Results for the four samples and their duplicates ranged in value from 30.98 to 52.12 percent water by weight. Results for sample S95T000807 exceeded the RPD target of 10\%. A triplicate analysis was performed, resulting in a determination of 39.79 weight percent water, compared to $41.04 \%$ and $33.26 \%$. The triplicate result does not appear in the summary tables, but is included in the raw data.

There was a significant variation in results between samples, with upper half samples being drier than lower half samples. All samples were well above the safety screening minimum of 17 weight percent. Three LMCS control standards were run for these TGA analyses and exhibited recoveries ranging from 96.77 to 99.70 percent, which were within the program's specified accuracy control limits of 90 to 110 percent.

\section{Total Alpha Activity}

Analyses for total alpha activity were performed on four samples (S95T000800, S95T000804, S95T000808, and S95T000812). Samples were prepared by fusion using procedure LA-549-141, Rev. C-2, and analyses were performed using procedure LA-508-101, Rev. D-2. A sample duplicate was performed on each sample. Due to the poor sample reproducibility and spike recovery, the samples were rerun. Rerun results for samples S95T000808 and $595 T 000812$ were retained, with the original results being rejected. However, the results of the rerun for samples S95T000800 and S95T000804 were less satisfactory than the original results (poorer spike recovery). Therefore, the original results for these samples were retained, and the results of the rerun were rejected.

Sample and duplicate results ranged from 0.55 to $2.83 \mu \mathrm{Ci} / \mathrm{g}$. The RPDs for samples $595 \mathrm{~T} 000800$ and $595 \mathrm{~T} 000804$ were $9.90 \%$ and $18.5 \%$, respectively The TCP 
WHC-SD-WM-DP-112, REV. 0

target value is $10 \%$. The RPDs for samples S95T000808 and S95T000812 were exceptionally high; $91.8 \%$ and $101 \%$ respectively. The chemist speculated that perhaps the duplicate samples were inadvertently mislabeled. If this assumption were correct, the sample and duplicate results would match much more closely (recalculated RPDs would be $4.33 \%$ and $16.8 \%$ ).

Two control standards were run, with recoveries of 92.57 and $91.89 \%$, within the TCP target of 90 to $110 \%$. A spike was performed on sample S95T000800, with a recovery of $68.4 \%$. This is outside of the TCP target recovery of 90 to $110 \%$. Spike recoveries for alpha have typically been below the target criterion, particularly when solids can be observed on the sample mount (solids will be self-shielding to some extent).

The above problems were considered, but deemed not significant enough to warrant another set of digestions and analyses since the results were far below the safety screening limit of $41 \mu \mathrm{Ci} / \mathrm{g}$ (highest sample result is only $7 \%$ of the limit).

\section{Density}

Characterization $\mathrm{Pl}$ ant Engineering requested a density determination on several auger samples. Their need was to verify that a Safety Analysis Report for Packaging (SARP-002) Timit on the volume of waste allowed to be shipped in an auger sampler was not exceeded. The density was determined by procedure L0-160-103, Rev. A-7. Subsamples were centrifuged in a tared, graduated centrifuge cone. Two samples were submitted for density determination, with the results being 1.72 and $1.51 \mathrm{~g} / \mathrm{mL}$. These results are presented in Tables 2 and 3.

REFERENCE Schreiber, R. D., 1995, WHC-SD-WM-TP-306, Revision 0, "Tank 241-C-203 Tank Characterization Plan, dated March 6, 1995. 
WHC-SD-HM-DP-112, REV. 0

SAMPLE DATA SUMMARY

6 
CORE NUMBER: $n / a$

TABLE 2

CORE NUMBER: $\mathrm{n} / \mathrm{a}$
SEGMENT H: $95-\mathrm{AUG}-020$

SEGMENT PORTION: $U$ Upper Half of Segment

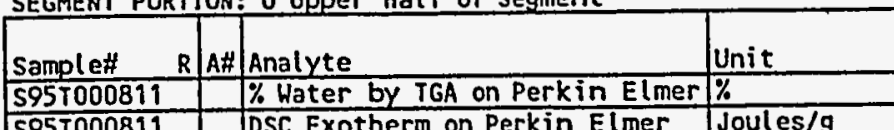

S95T000811 \% Water by TGA on Perkin Elmer

595T000811 DSC Exotherm on Perkin Elmer Joules/g

S95T000811 DSC Exotherm Dry Calculated

\begin{tabular}{l|l|l}
5957000812 & $F$ & Alpha of Digested Solid \\
\hline
\end{tabular}

None None

Action limits Lower $\frac{\text { Upper }}{110.000}$ oules/g Dry $-999.000 \quad \% 489040$

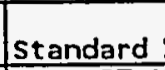
97.45

97.45

Blank

\begin{tabular}{r|r|r}
\hline lank & Result & Dup \\
\hline $\mathrm{n} / \mathrm{a}$ & 30.98 & \\
\hline $\mathrm{n} / \mathrm{a}$ & 34.91 & \\
\hline $\mathrm{n} / \mathrm{a}$ & & \\
\hline
\end{tabular}

\begin{tabular}{r|r} 
Duplicate & \\
\hline 31.78 & \\
\hline 33.19 &
\end{tabular}

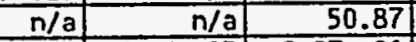
\begin{tabular}{r|r|r|r|r|}
$\mathrm{n} / \mathrm{a}$ & $\mathrm{n} / \mathrm{a}$ & 50.87 & 48.37 & 49.62 \\
\hline 97.89 & $<4.46 \mathrm{e}^{-03}$ & $8.87 \mathrm{e}-01$ & $2.71 \mathrm{e}+0$ & $1.80 \mathrm{e}+00$ \\
\hline
\end{tabular}

48.37 -999.000 \% 4.1 .010 \begin{tabular}{|r|r|r|r|r|}
\hline Average & RPD \% & Spk Rec \% & Det Limit & Count Err\% \\
\hline 31.38 & 2.55 & $n / a$ \\
\hline
\end{tabular} \begin{tabular}{l|l|l}
31.38 & 2.55 \\
\hline 34.05 & 5.05
\end{tabular} \begin{tabular}{l|l|}
\hline 34.05 & 5.05 \\
\hline 49.62 & 5.04 \\
\hline
\end{tabular} $n / a$ $n / a$ $n / a \quad n / a$ $n / a$

Lower Half of Segment: L Lower Half of Segment

Lower Half of Segment: L Lower Half of Segment
\begin{tabular}{|l|l|l|}
\hline Sample\# & A\# & Analyte \\
\hline S95T000807 & \% Water by TGA on Perkin Elmer \\
\hline S95T000807 & DSC Exotherm on Perkin Elmer \\
\hline S950u E
\end{tabular}

Action Limits

in

\begin{tabular}{l|l|} 
Lower & Upper \\
\hline
\end{tabular}

standard

T. 01 n/a $1.00 \mathrm{e}-02$

$n / 2$

DSC Exotherm on Perkin Elmer Joules/g

595T000807

DSC Exotherm Dry Calculated Joules/g Dry

None

None None tandard \%

\begin{tabular}{r|r} 
Blank & R \\
\hline $\mathrm{n} / \mathrm{a}$ & \\
\hline $\mathrm{n} / \mathrm{a}$ & $\mathrm{n} / \mathrm{a}$ \\
\hline
\end{tabular}

Result 41.04
20.36 $\frac{\text { Duplicate }}{\frac{33}{30}}$

\begin{tabular}{l|l} 
cate & Av \\
\hline 3.26 & \\
\hline 19.9 & \\
\hline 0.82 &
\end{tabular}

Average uci/g

$91.89<4.46 \mathrm{e}-03$

2.830

\begin{tabular}{r|r|r|}
\hline $.05 e+0$ & $1.94 e+00$ & 91.8
\end{tabular}

\begin{tabular}{l|l|}
\hline 37.15 & 20.9 \\
\hline 19.73 & 6.39 \\
\hline 31.84 & 6.38 \\
\hline $4 e+00$ & 91.8 \\
\hline
\end{tabular}

\begin{tabular}{l|l}
$\%$ & Spk Re \\
\hline 9 & \\
\hline 39 & \\
\hline 8 &
\end{tabular}

\begin{tabular}{|c|c|c|}
\hline ec \% & Det Limit & Count Err\% \\
\hline$n / a$ & $n / a$ & $n / a$ \\
\hline$n / a$ & $n / a$ & $n /$ \\
\hline$n / a$ & $n / a$ & $\mathrm{n} /$ \\
\hline$n / a$ & $1.10 \mathrm{e}-02$ & 3.0 \\
\hline
\end{tabular}

\section{$\therefore \therefore \therefore \quad \Rightarrow$ Limit violated \\ $\Rightarrow$ Selected Limit}

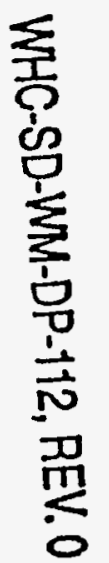


ORE NUMBER: $n / a$

TABLE 3

EGMENT \#: 95-AUG-021

EGMENT PORTION: U Upper Half of Segment

\begin{tabular}{|c|c|c|c|}
\hline mple\# & R $\mid$ A\# & Analyte & Unit \\
\hline $95 T 000798$ & & Bulk Density of Sample & $\mathrm{g} / \mathrm{mL}$ \\
\hline 957000799 & & $\%$ Water by TGA using Mettler & $\%$ \\
\hline $95 T 0$ & & DSC Exotherm Dry Calculated & Joules/g or \\
\hline $95 T 000799$ & & DSC Exotherm using Mettler & Joules/g \\
\hline 957000800 & $\mathrm{~F}$ & Alpha of Digested Solid & $\mathrm{UCi} / \mathrm{g}$ \\
\hline
\end{tabular}

\begin{tabular}{|c|c|c|c|c|c|c|c|c|c|c|c|}
\hline & Action & Limits & & & & & & & & & \\
\hline & Lower & Upper & Standard \% & Blank & Result & Duplicate & Average & RPD \% & Spk Rec \% & Det Limit & Count Err\% \\
\hline & None & None & $n / a$ & $\mathrm{n} / \mathrm{a}$ & 1.720 & $n / a$ & $n / a$ & $n / a$ & $n / a$ & $5.00 e-01$ & $n / a$ \\
\hline & 7\%.600: & 110.000 & 99.54 & $\mathrm{n} / \mathrm{a}$ & 33.67 & 36.66 & 35.16 & 8.50 & $n / a$ & $n / a$ & $n / a$ \\
\hline$\frac{\text { les/g Dry }}{\text { jles/g }}$ & -999.000 & 681,010 & $\mathrm{n} / \mathrm{a}$ & $\mathrm{n} / \mathrm{a}$ & 66.02 & 64.32 & 65.17 & 2.61 & $\mathrm{n} / \mathrm{a}$ & $\mathrm{n} / \mathrm{a}$ & $\mathrm{n} / \mathrm{a}$ \\
\hline ules/g & -999.000 & $483 \times 109$ & $\frac{99.82}{0.57}$ & $n / a$ & 42.80 & $\frac{41.7}{1.50}$ & $\begin{array}{r}42.25 \\
1520+00\end{array}$ & $\frac{2.60}{9.90}$ & $\frac{n / a}{68.40}$ & $\frac{n / a}{7.00 e-03}$ & $\frac{n / a}{3.1}$ \\
\hline
\end{tabular}

Lower Half of Segment: L Lower Half of Segment

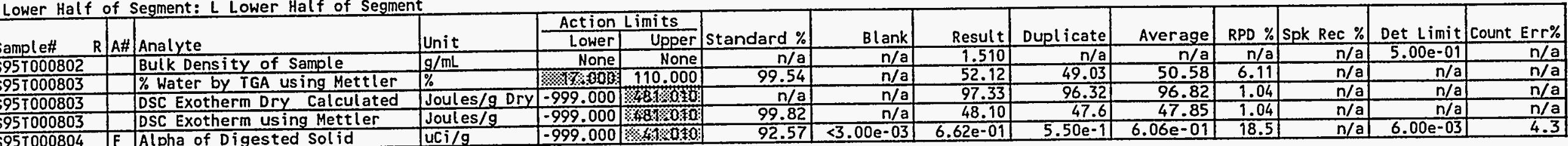

\begin{tabular}{|l|l|l|}
\hline 957000803 & & DSC Exotherm using Mettlel \\
\hline 957000804 & F & Alpha of Digested Solid \\
\hline
\end{tabular} $u \mathrm{Ci} / \mathrm{g}$

$\Rightarrow$ Limit violated

$\Rightarrow$ Selected Limit 
WHC-SD-WM-DP-112, REV. 0

PHOTOGRAPHS 


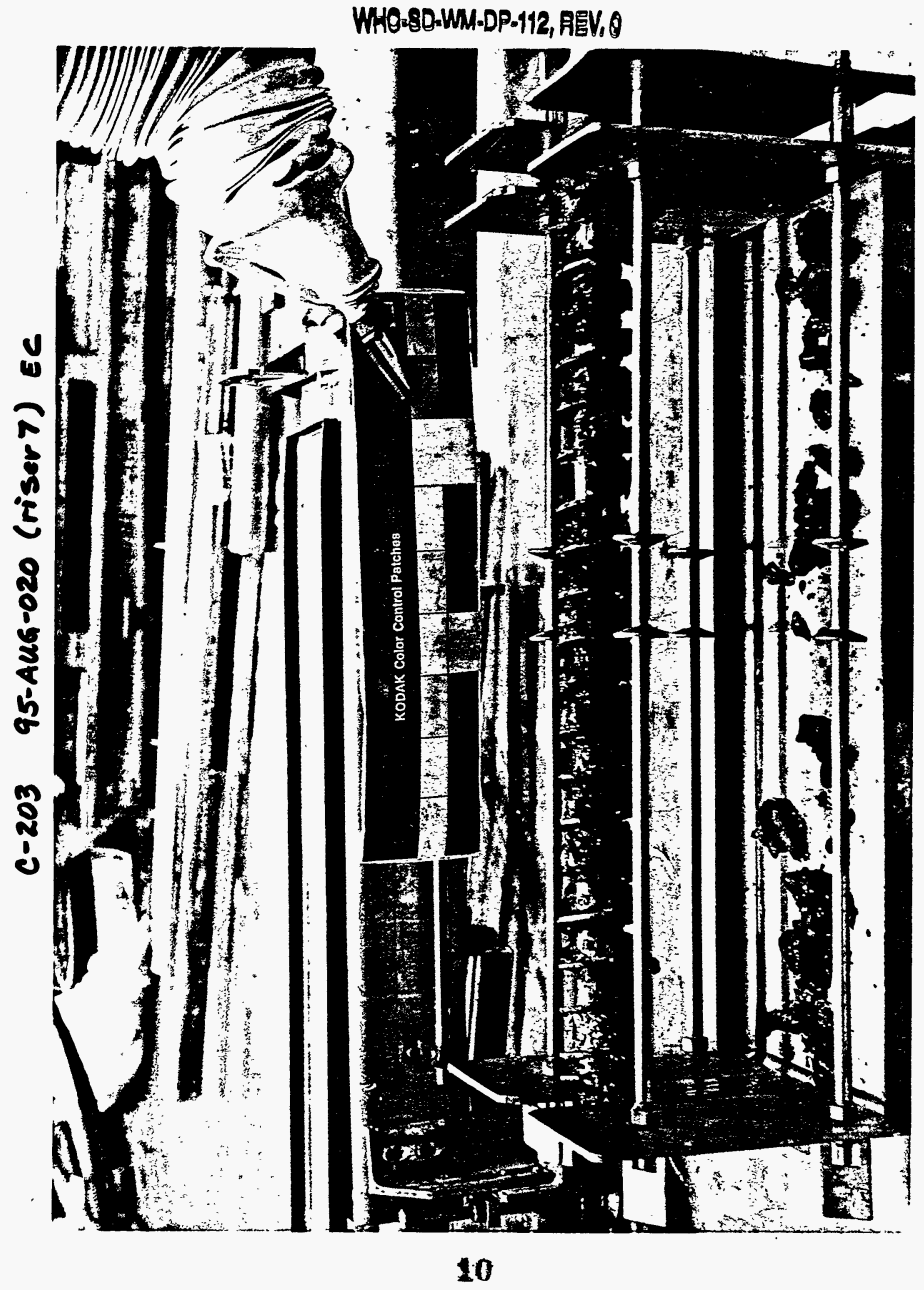


WHC-SD-WM-DP-112, REV. 0

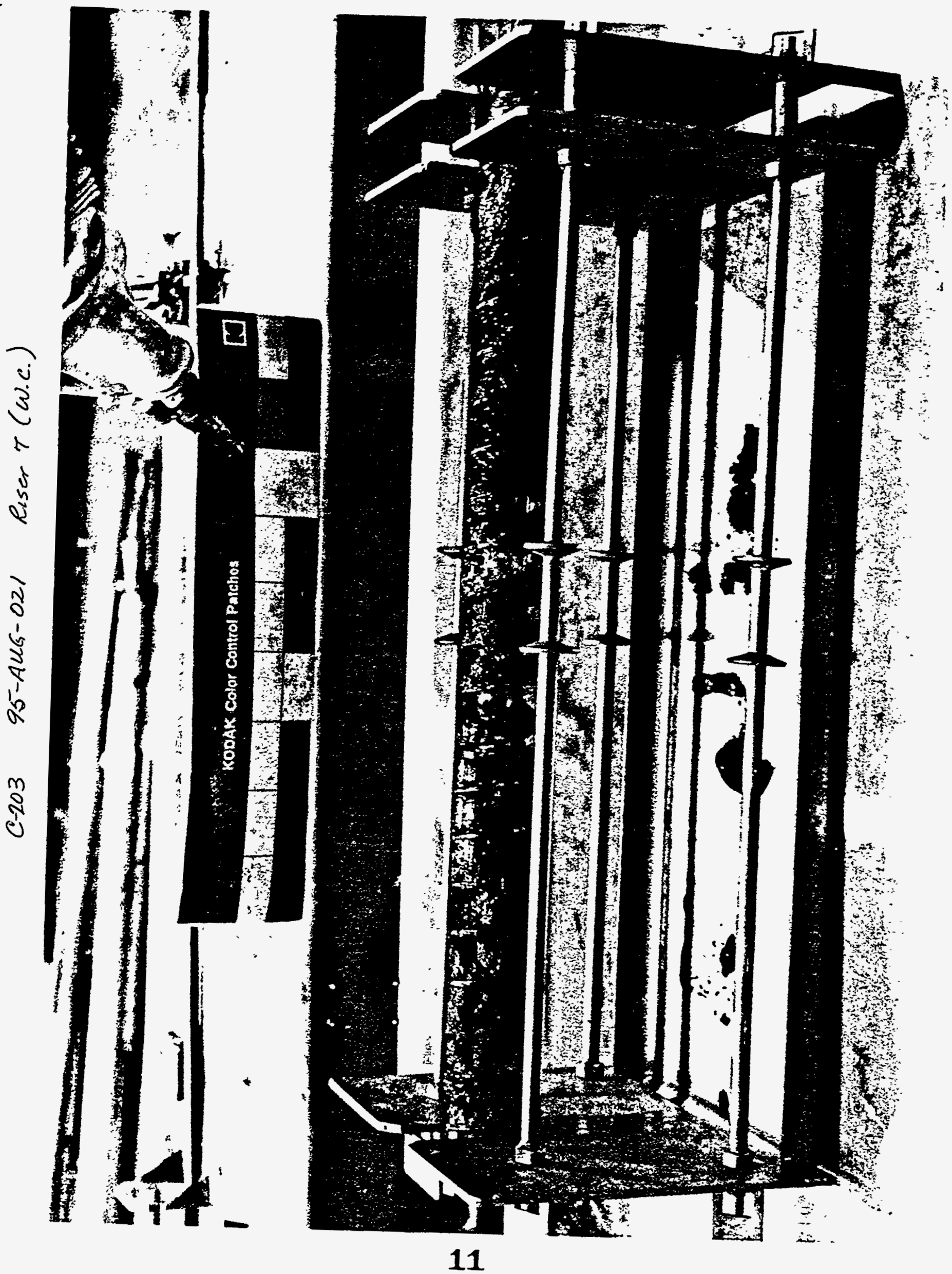


WHC-SD-WM-DP-112, REV. 0

CHAIN OF CUSTODY FORHS 


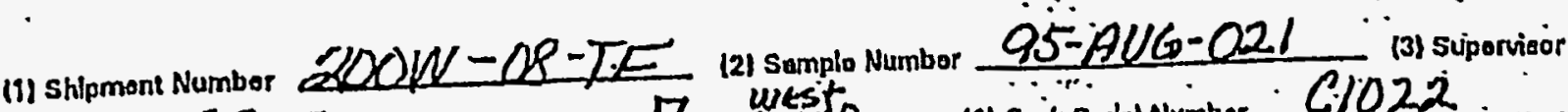
R.J. Prazwik/373-7751

(4) Tank 2203 (6) Rlear 7 cusst.

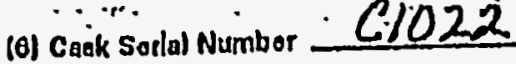

\section{Rodlation Survoy Data:}

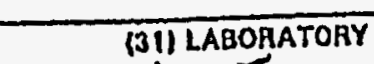

Ovat Top Dose Rate

Sldo Dono Ralo

Battom Done flate

Smearablo Contamination

$\frac{\frac{20.5}{175^{\text {FIELD }}}}{\frac{20.5}{20}}$

L K $\mathrm{K}$

$C$

ncr. $\frac{72 \text { Bdelenters }}{\text { istonatural }}$ (Bota-Camma) istonatural

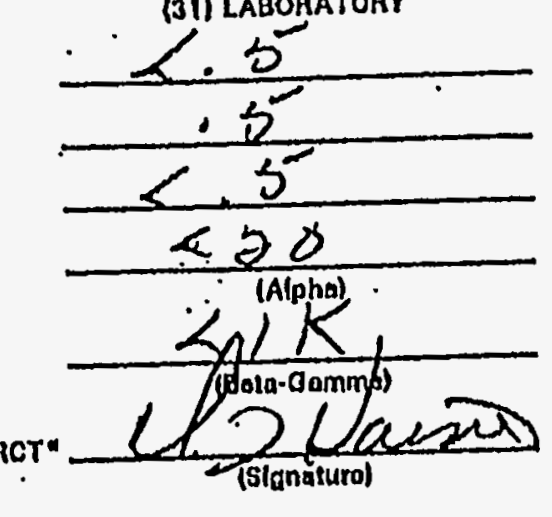

\section{(8) Shipmone Dososiptlon}

A. Wark Packego Numbor

B. Cark Soal Numbor

C. Osto and TImo Samplo

Romovad from Tank

D. Expoctod Liquld Contont

E. Expootod solld Contont

F. Dosa Haio Through Dill Strivia

a. Expootod Sampla Langth
ES.95-00011-0

H1029

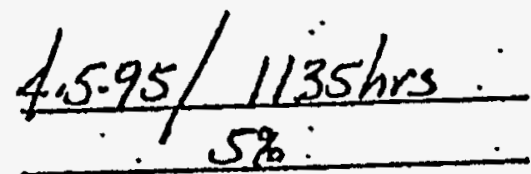

958

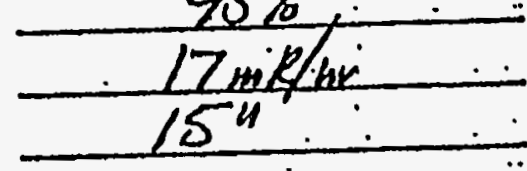

\section{(9) INFORMATION (Inolude etalomone ol lebaratory soate to bo parformod.)}

.

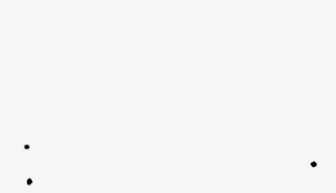

129) Seal InLoor Upon Roodipl?. Q
Shlpment No. Bros Q DNo

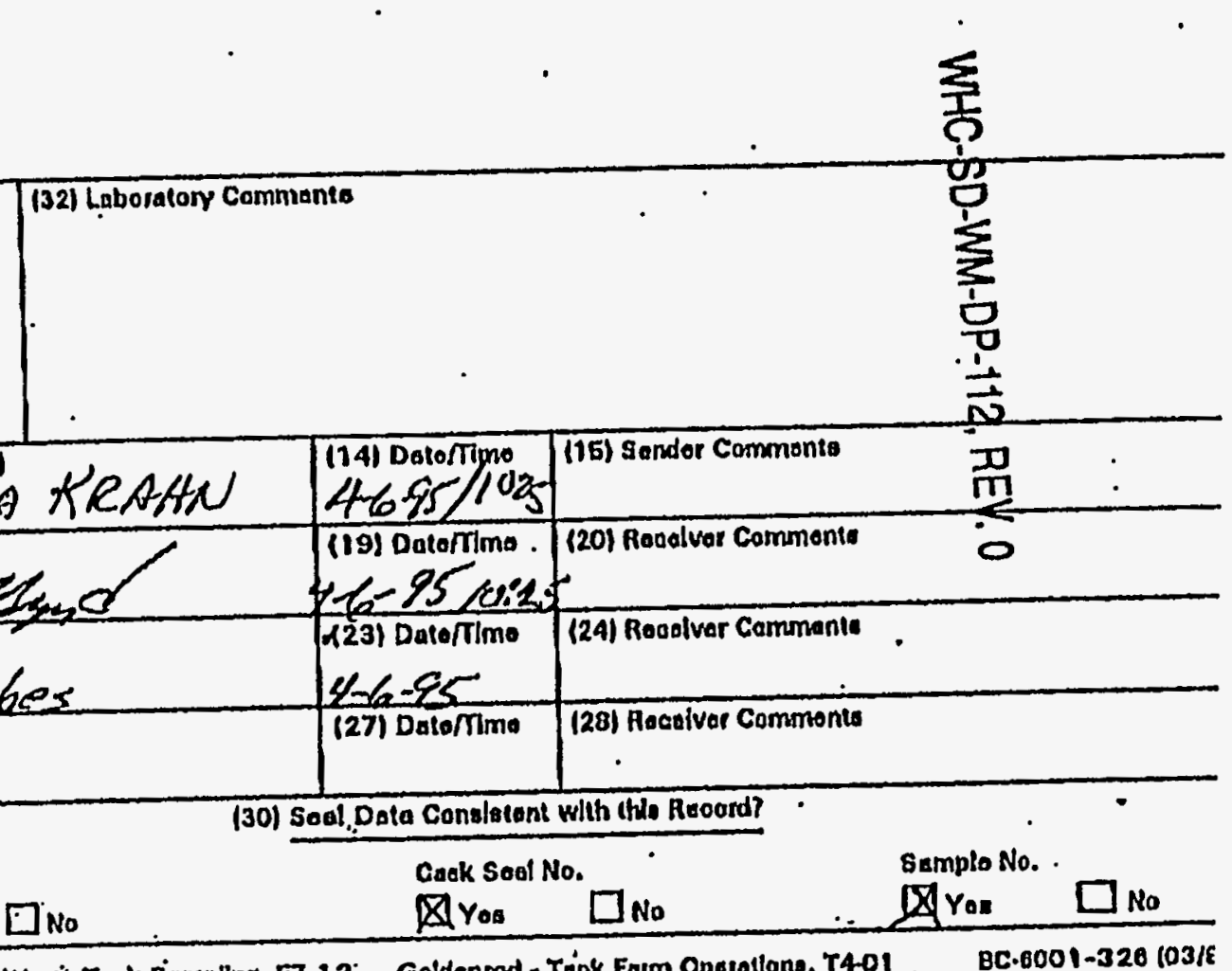




\section{CHAIN-OF-CUSTODY RECOAD FOR AUGER SAMPLING}

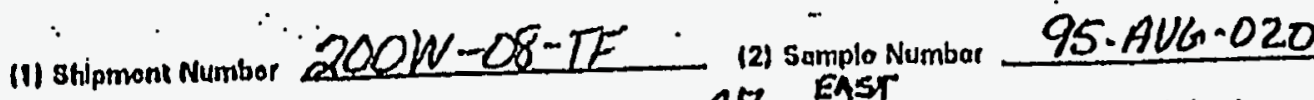
(3) Suparvisar

R.J. Penznik/323-725

(4) Tank 2203

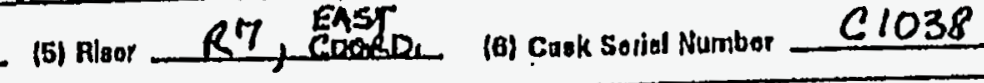

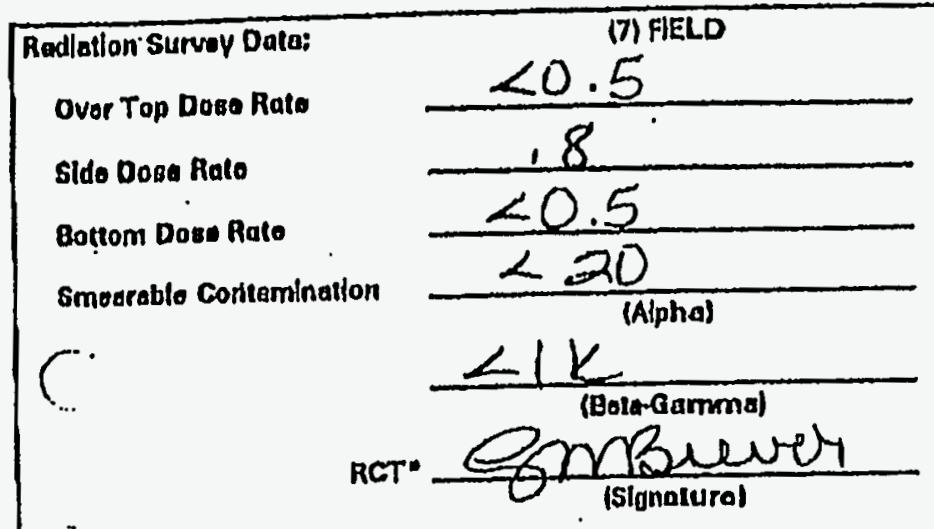

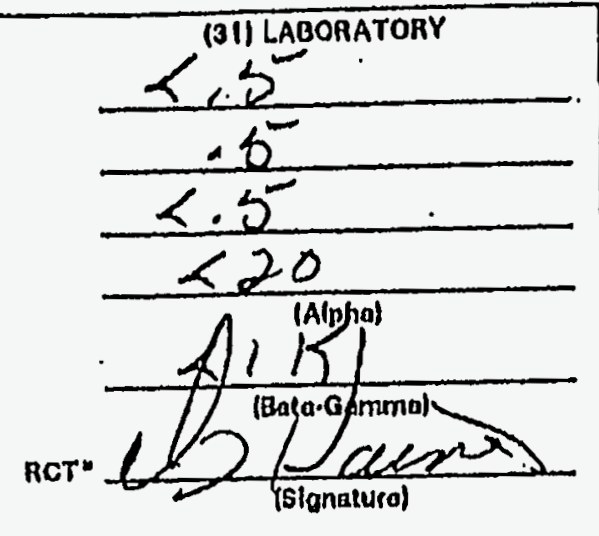
8) Shlpmonc Davorlption
A. Work Paokego Number
B. Ceak Sool Numbor
C. Dolo and TImo Samplo.
Homovod fram Tonk
D. Expootod Llquid Contons
E. Expootod Solid Content
F. Dogo Aato Through Disil Sising
O. Exprotod Somplo Longth

Es-95-00011-0 W1028

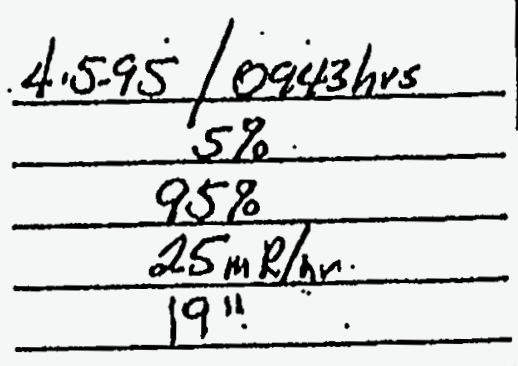

(9) INFORMATION Inoluda o(stamant of leboratory losto to ba porformod.)

C.

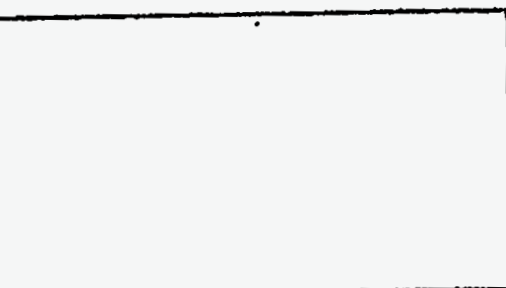

\section{(11) Polnt ol Oilgln Ehst (12) Destination C203 R/7 Coom $2225 L A B$ (1+74 Rellngulohod gy (s)en and PRINT)} D) lich DAKRAAN

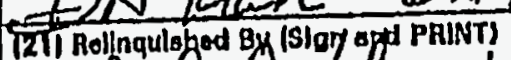
Jubul do Nelino Jubal O Helms 125) Rallnqulshod By (SIgn and PRINT)

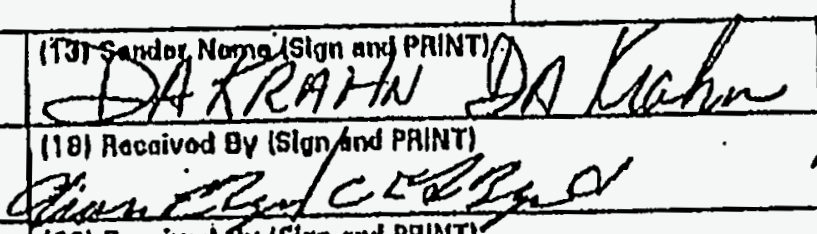

(22) Hoggivod Gy (Sion und PRINT)

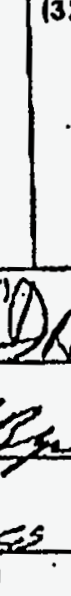
(20) Hoouivod By (sion and PRINT)
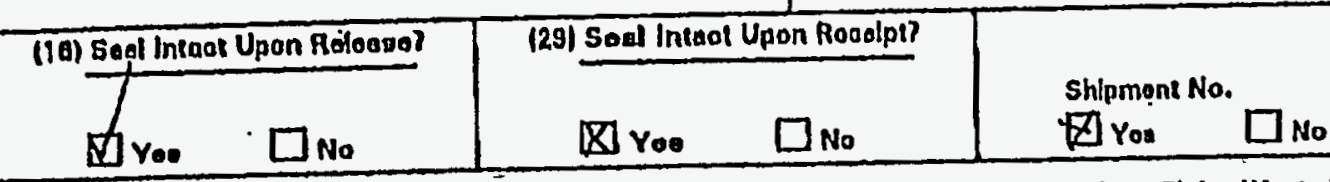

¿hipmọnt No. $\square$ No

- (30) Sosl Data Condatent with shls Racord?

(14) DatorTim/ (15) Sondor Commonto $4-6.95 / 10_{3}$ (19) Datorfitiono (20) Aacolvar Commento

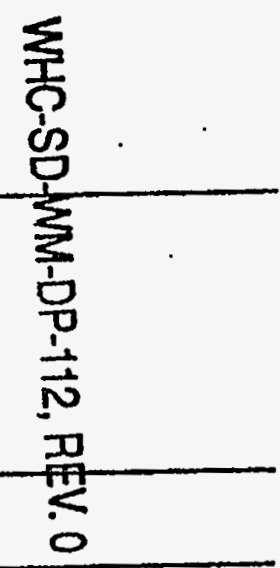
$-6-75,24$ (23) Delootimo 1241 Racolvar Commonto $44-6-8+110$ $4-x-851120$ (28) Rlocuivar Commonto

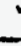

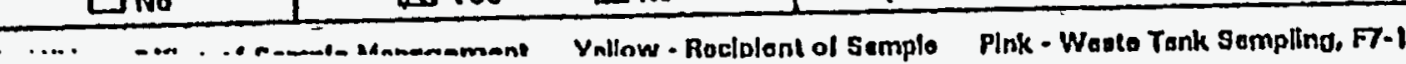
Cask Soal No. $\square$ No

WYos. Gotdaniod - Tank Farm Oparations, T401 . BC-6001.326103\% 
WHC-SD-WH-DP-112, REV . 0

SAMPLE ANALYSES RESULTS 


\section{LABCORE Data Entry Template for Worklist\# 1005}

Analyst: $\quad \sum C \quad$ Instrument: BA000_ Book \# NA

Method: LO-160-103 Rev/Mod A-7

Worklist Comment: C-203 95-AUG-021 Riser 7 Extrusion

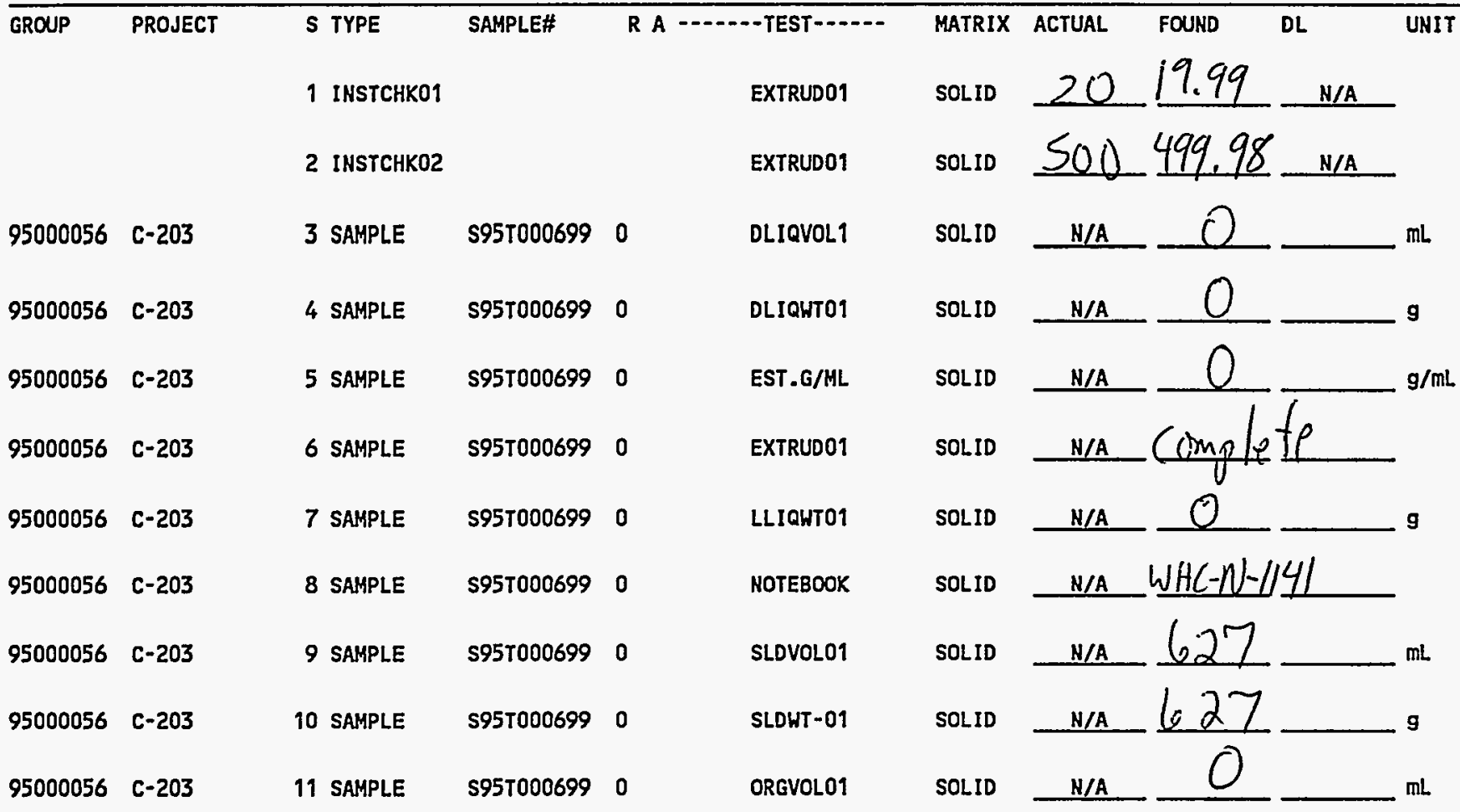

Final page for worklist \# 1005

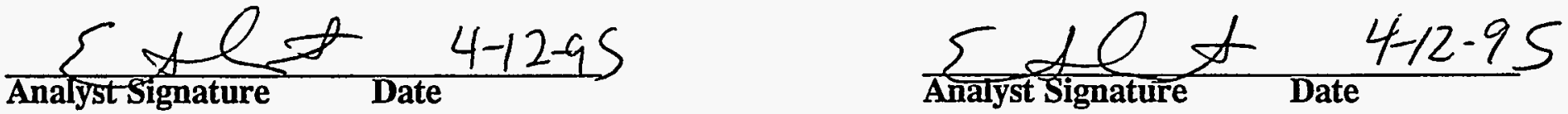

Data Entry Comments:

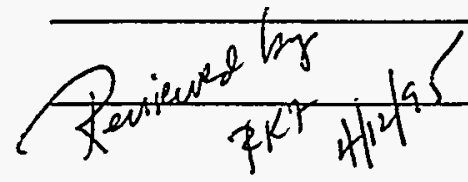

Units shown for $Q C$ (SPK \& STD) may not reflect the actual units. $D L=$ Detection Limit, $S=$ Worklist Slot Number; $R=$ Replicate Number, $A=$ Aliquot Code. 


$$
\text { WHC-SD-wm-DP-112, Rw. }
$$

Analyst: $\quad \sum C$ Instrument: BA000_ Book \# VA

Method: LO-160-103 Rev/Mod A.7

Worklist Comment: C-203 95-AUG-020 Riser 7 Extrusion

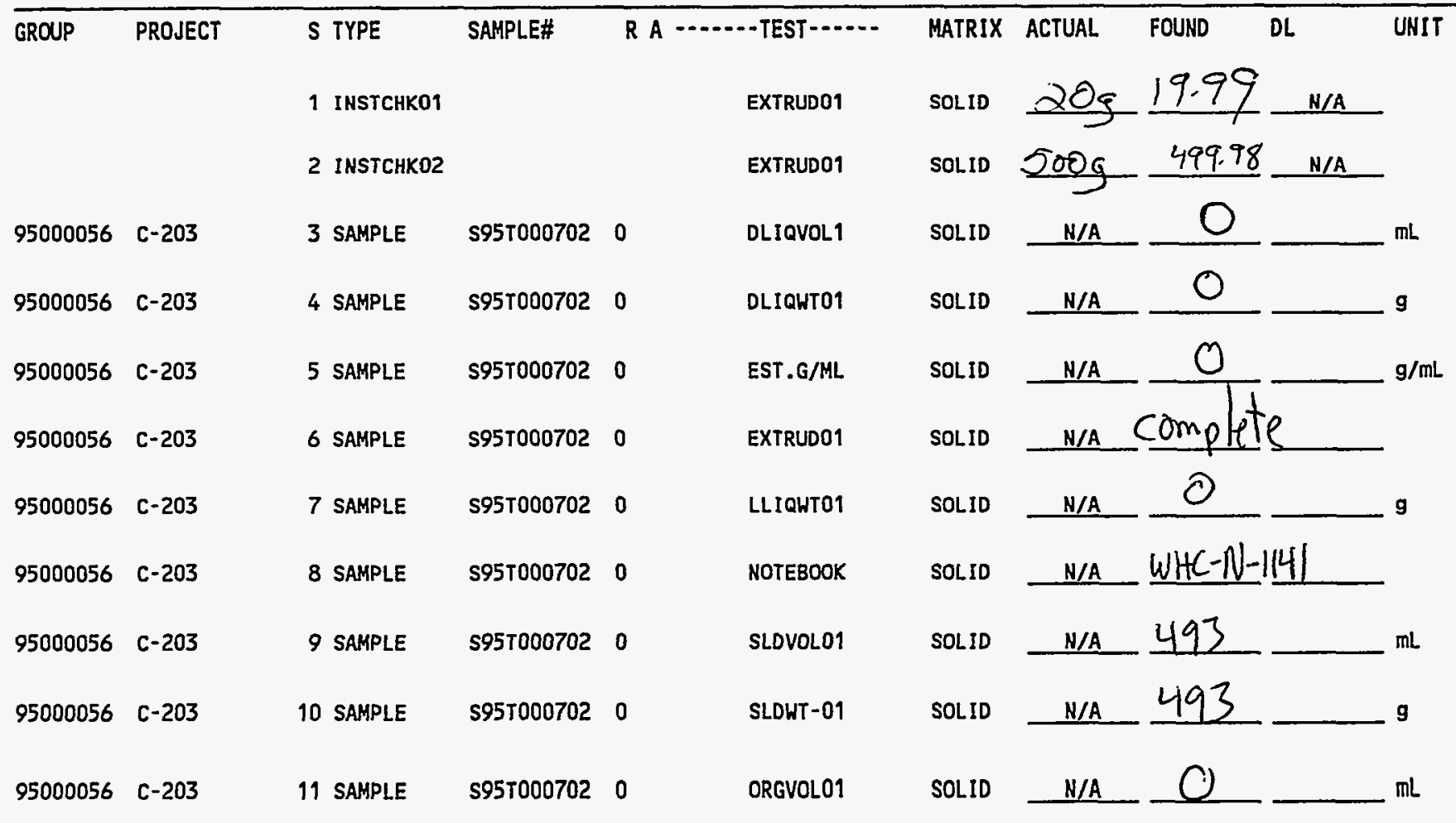

\section{Final page for worklist \# 1006}

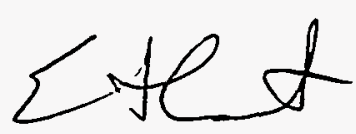

Analyst Signature

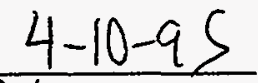

Date

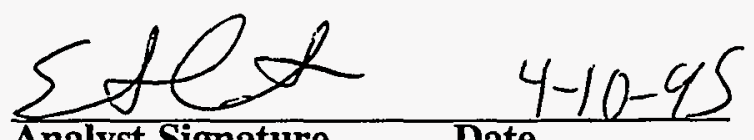

Analyst Signature
Date

Data Entry Comments:

\section{Revieured by

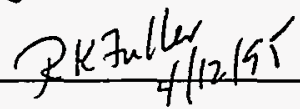

Units shown for $Q C$ (SPK \& STD) may not reflect the actual units. $D L=$ Detection Limit, $S=$ Worklist Slot Number, $R=$ Replicate Number, $A=$ Aliquot Code. 


\section{LABCORE Data Entry Template for Worklist\# 1276}

Analyst: $\quad S C \quad$ Instrument: BA000_ Book \# $N A$

Method: L0-160-103 Rev/Mod A - 7

Worklist Comment: U-202, C-101, and C-203 Archives

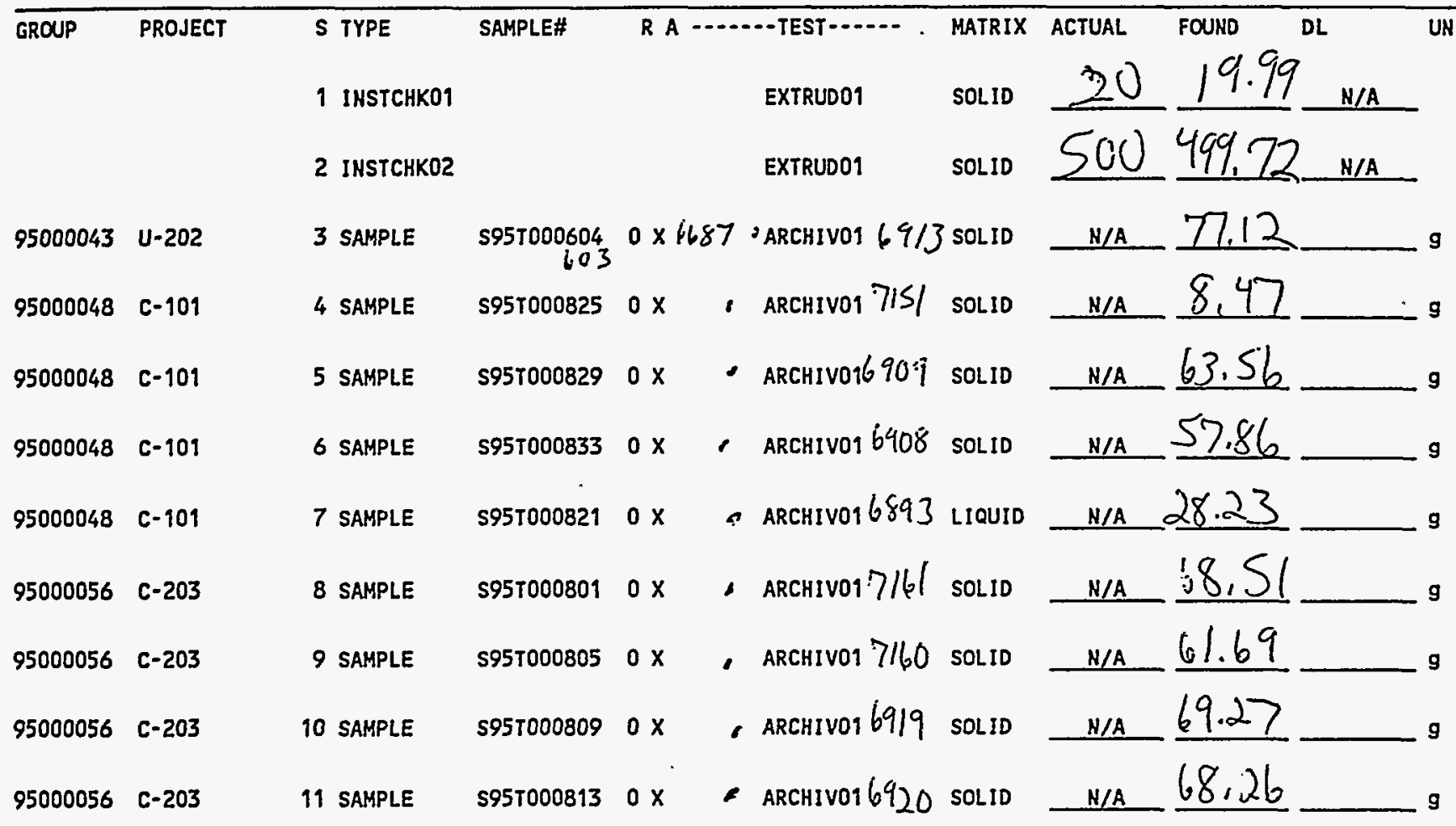

\section{Final page for worklist \# 1276}
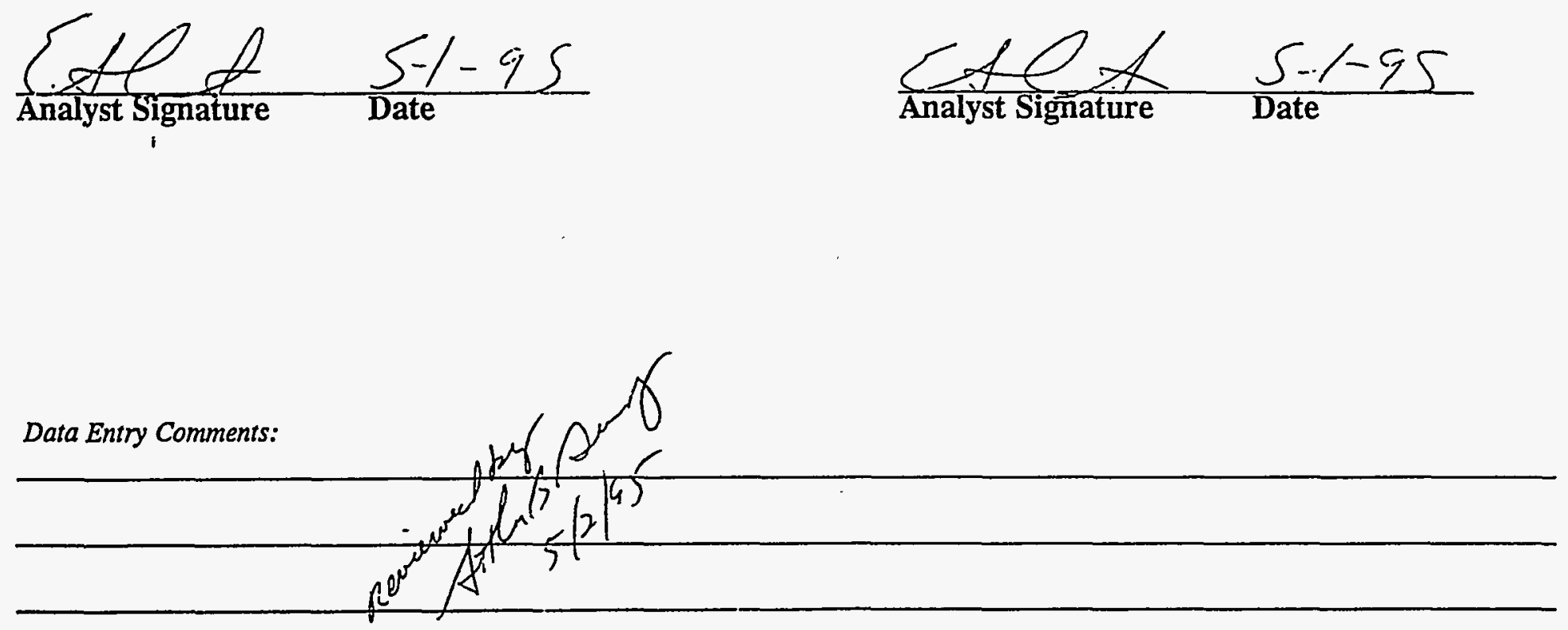

Units shown for $Q C$ (SPK \& STD) may not reflect the aciual units. $D L=$ Detection Limit, $S=$ Worklist Slot Number, $R=$ Replicate Number, $A=$ Aliquot Code. 


\section{LABCORE Data Entry Template for Worklist\# 1322}

Analyst: ABS $\quad$ Instrument: BA000_ Book\# $\sim A$

Method: LO-160-103 Rev/Mod A-Z

Worklist Comment: C-101 and C-203 Bulk Density Determinations

\begin{tabular}{|c|c|c|c|c|c|c|c|c|c|c|}
\hline \multirow[t]{3}{*}{ GROUP } & \multirow[t]{3}{*}{ PROJECT } & $S^{-1 Y P E}{ }^{\circ}$ & SAMPLE\# & \multicolumn{2}{|c|}{$\begin{array}{llll}\text { R A } & - & - & \text {-TEST } \cdots \cdots \\
\end{array}$} & MATRIX & ACTUAL & FOUND & $\overline{D L}$ & UNIT \\
\hline & & 1 INSTCHKOT & & & EXTRUD01 & SOLID & 20 & $14,5 \xi$ & N/A & \\
\hline & & 2 INSTCHKO2 & & & EXTRUD01 & SOLID & $5 \theta \theta$ & $\xi 5$ & N/A & \\
\hline 95000056 & $c-203$ & 3 SAMPLE & S95T000798 & 06781 & BLKDENO1 & SOLID & N/A & $\frac{1.72}{1.1}$ & & g/mL \\
\hline 95000056 & $c-203$ & 4 SAMPLE & S95T000802 & 06780 & BLKDEN01 & SOLID & N/A & & $5 / 4 / 95$ & $\mathrm{~g} / \mathrm{mL}$ \\
\hline 95000048 & $c-101$ & 5 SAMPLE & S95T000826 & 06936 & BLKDENOI 104 & SOLID & N/A & 1.84 & & $g / \mathrm{mL}$ \\
\hline 5000048 & $c-101$ & 6 SAMPLE & S95T000830 & 06937 & BLKDENO1 104 & SOLID & & 1.73 & & \\
\hline
\end{tabular}

\section{Final page for worklist \# 1322}
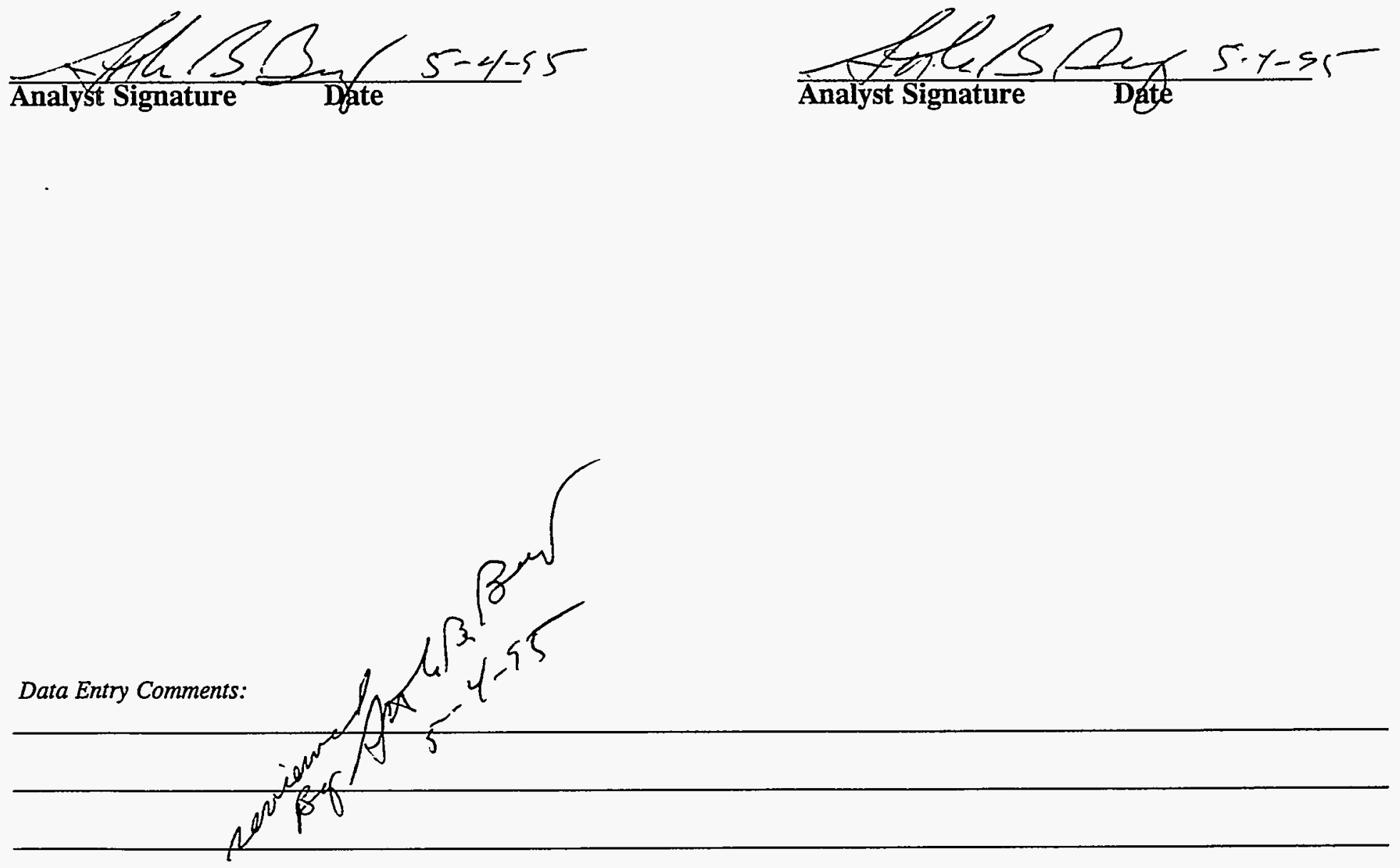

Units shown for $Q C$ (SPK \& STD) may not reflect the actual units. $D L=$ Detection Limit, $S=$ Worklist Slot Number, $R=$ Replicate Number, $A=$ Aliquot Code. 
WHC-SD-Wur-DP-112, Rew.o

worklistrpt Version 2.0 02/21/95

Page: 1

04/19/95 06:45

LABCORE Data Entry Template for Worklist\# 1173

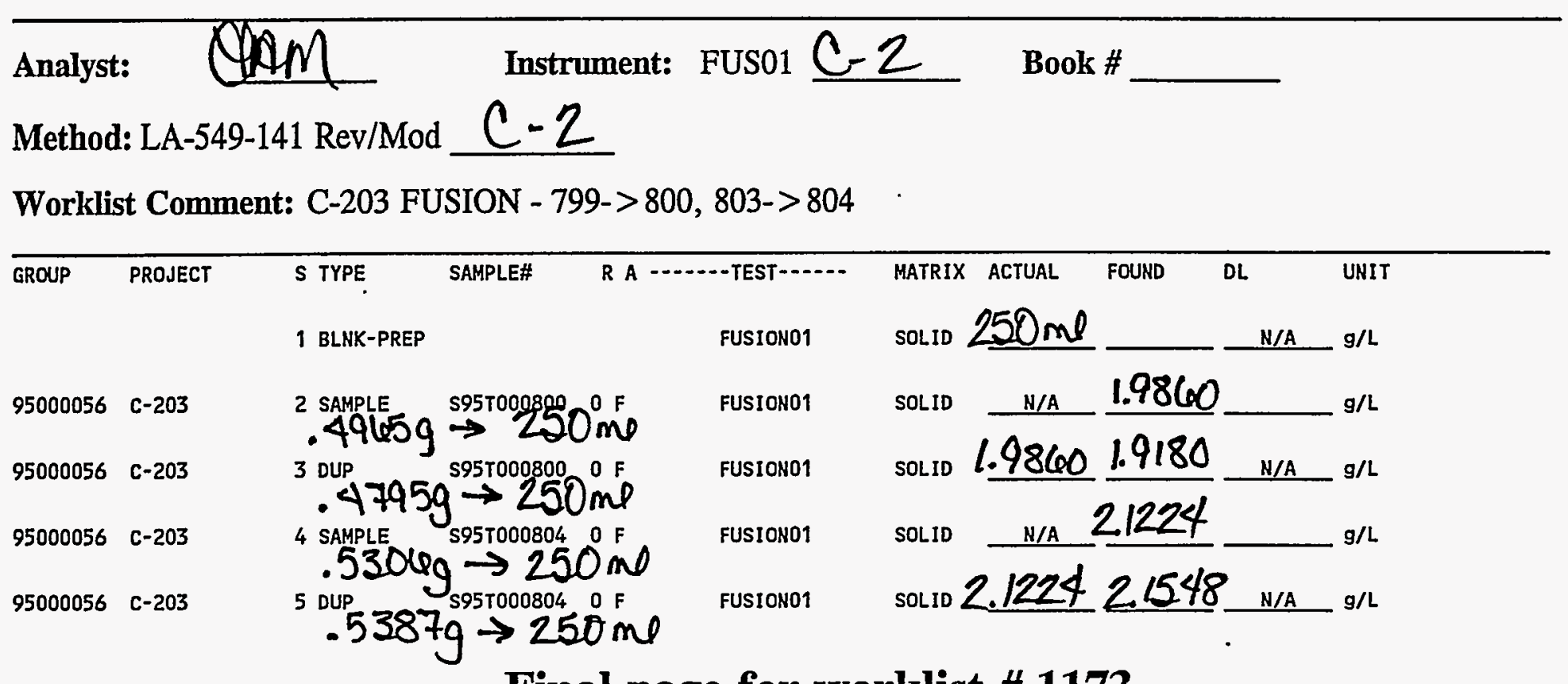

Final page for worklist \# 1173

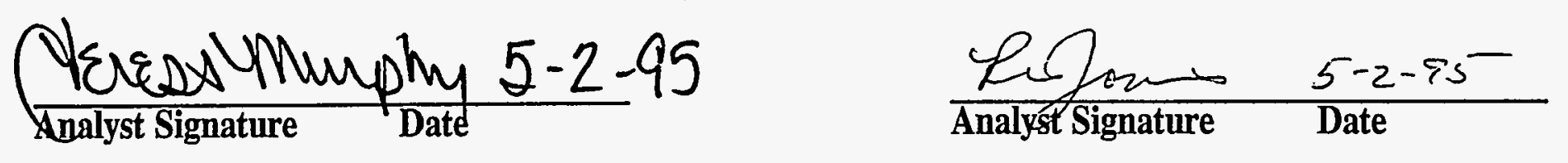

Datantry Comments: alienated 200/100/100. Samples \& cups have $<.190$ Dories. Dose nate is 2 mRad/hr HPT is Panel

Bastard Stiffen II 5-2-95 Nones

Units shown for $Q C$ (SPK \& STD) may not reflect the actual units. $D L=$ Detection Limit, $S=$ Worklist Slot Number, $R=$ Replicate Number, $A=$ Aliquot Code.

20 
worklistrpt Version 2.0 02/21/95

$$
\text { WHC SD-Wm-DP-112, Rus }
$$

Page: 1 0.4/19/95 06:47

LABCORE Data Entry Template for Worklist\# 1174

Analyst: $\operatorname{lm}$ Instrument: FUS01 AL 11066 Book \#

Method: LA-549-141 Rev/Mod C-Q

Worklist Comment: C-203 FUSION - 807-> 808, 811->812

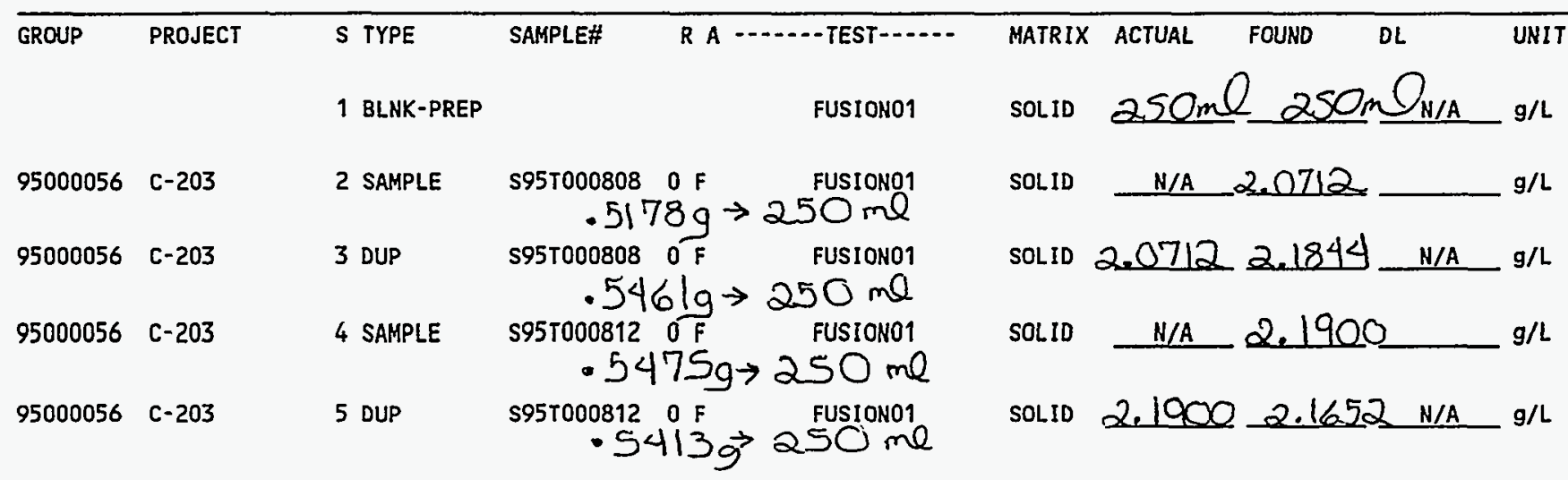

Final page for worklist \# 1174
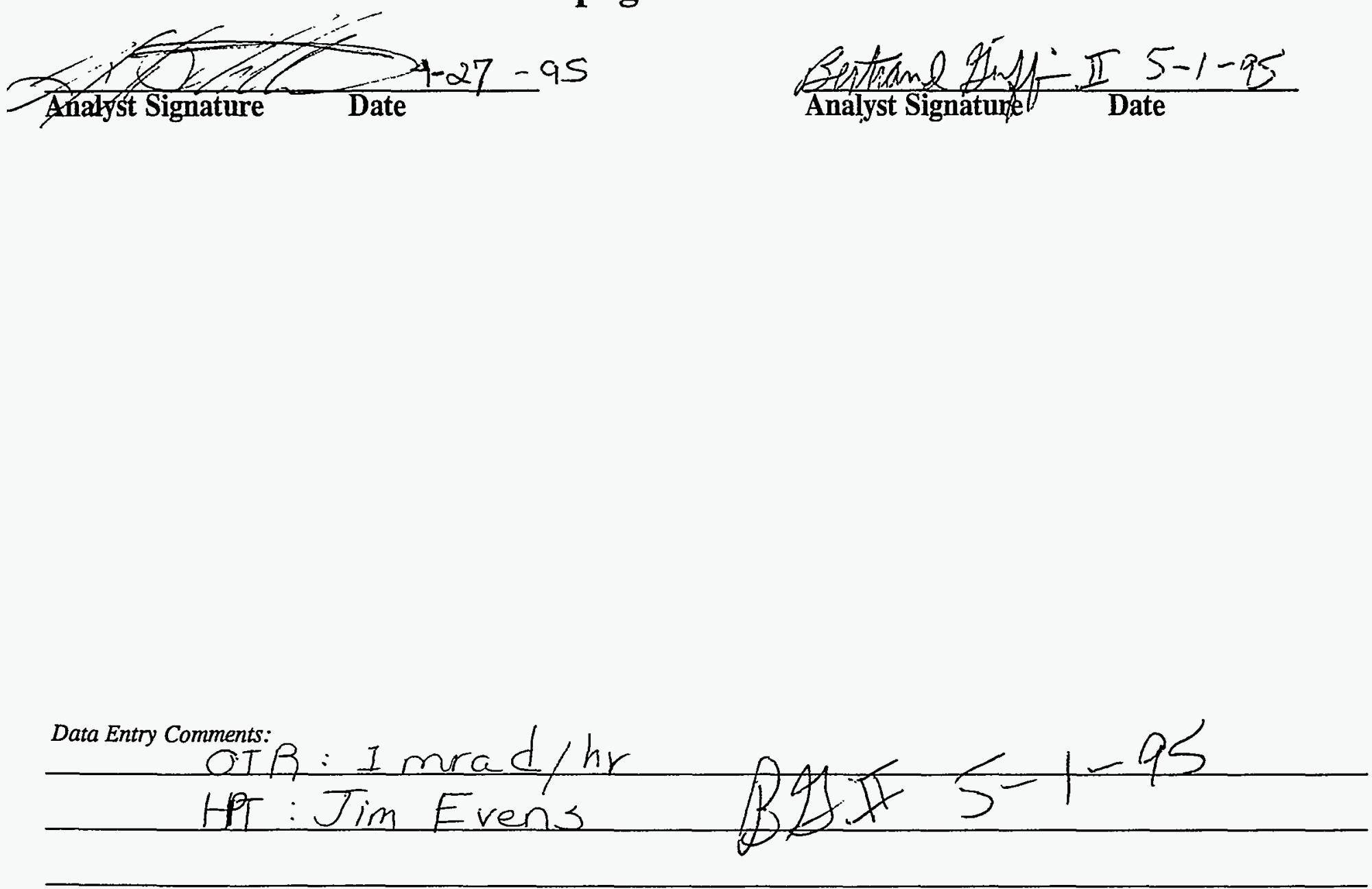

Units shown for QC (SPK \& STD) may not reflect the actual units. $D L=$ Detection Limit, $S=$ Worklist Slot Number, $R=$ Replicate Number, $A=$ Aliquot Code.

21 


\section{LABCORE Data Entry Template for Worklist\# 1216}

Analyst: $\quad$ Lhm $\cdot$ Instrument: DSC01

Book \# $12 N 14-A$

Method: LA-514-113 Rev/Mod $\beta-1$

Worklist Comment: Please run C-203 DSC under N2. bdv

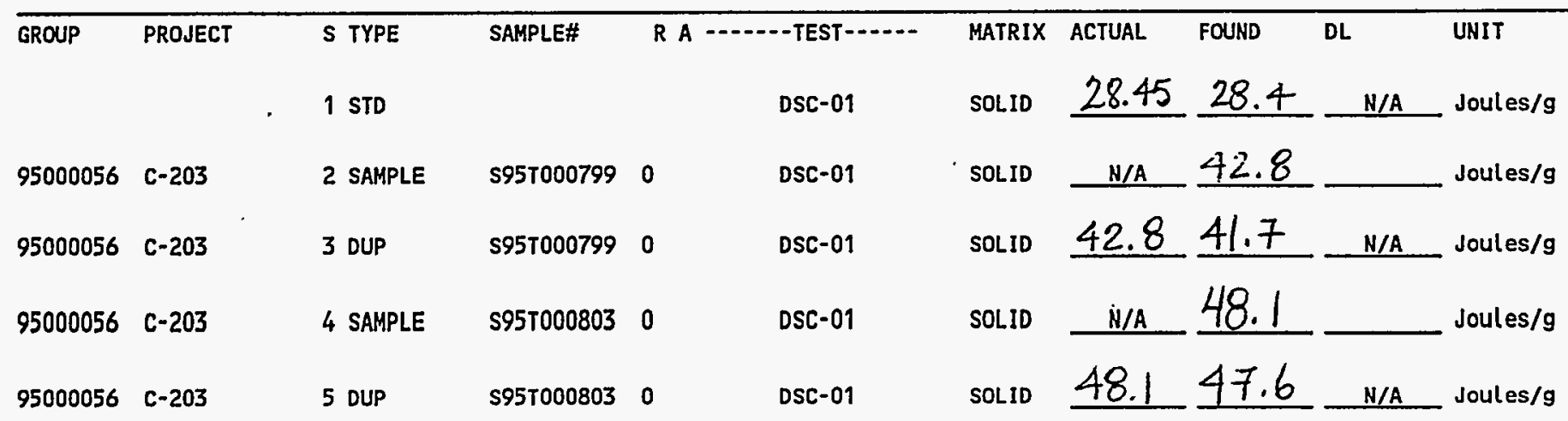

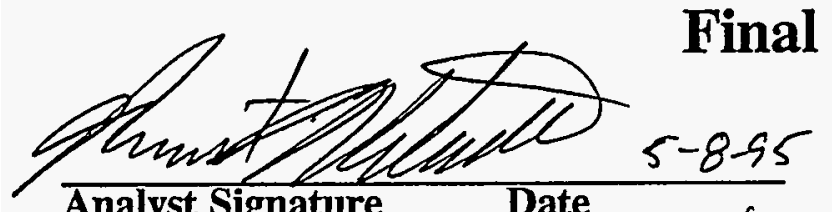

Analyst Signature Date

Verified by Blandina Valenzuela $5 / 9 / 95$

Final page for worklist \# 1216

$\$ 951000799$ - produced two indotherms one at $106.2^{\circ} \mathrm{C}$ with a deltatt of $845.5 \mathrm{~J} / \mathrm{g}$ and the second at $283.2^{\circ} \mathrm{C}$ with a delta $H$ of $43.7 \mathrm{~J} / \mathrm{g}$.

Data Entry Comments:

$\$ 957000803$ produced two endotherms, one at $112.8^{\circ} \mathrm{C}$ with a deeta $H$ of $761.8 \mathrm{~J} / \mathrm{g}$ and the second at $283.1^{\circ} \mathrm{C}$ with a dectaH of $48.1 \mathrm{~J} / \mathrm{g}$.

Units shown for $Q C$ (SPK \& STD) may not reflect the actual units. $D L=$ Detection Limit, $S=$ Worklist Slot Number; $R=$ Replicate Number, $A=$ Aliquot Code. 


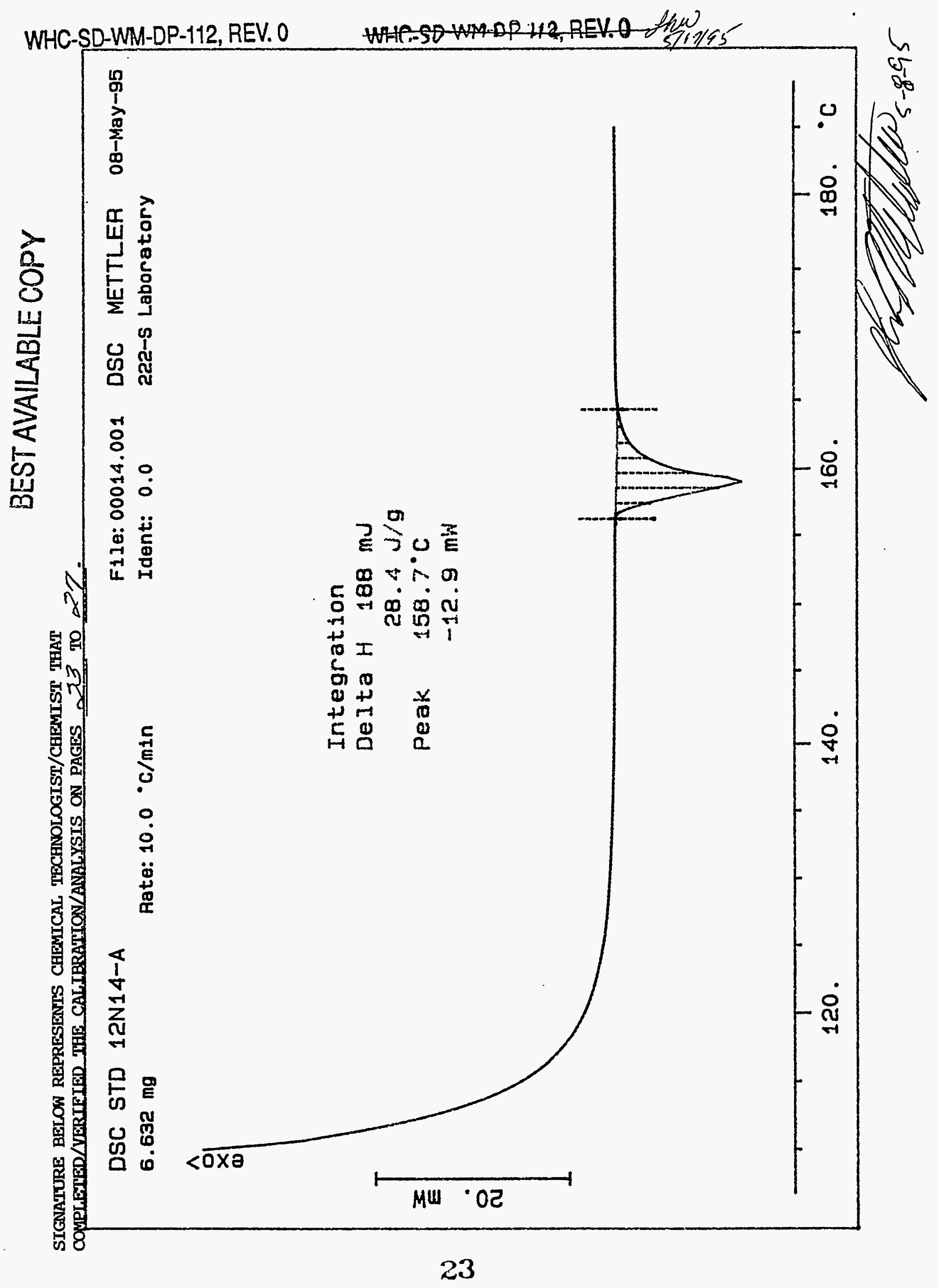




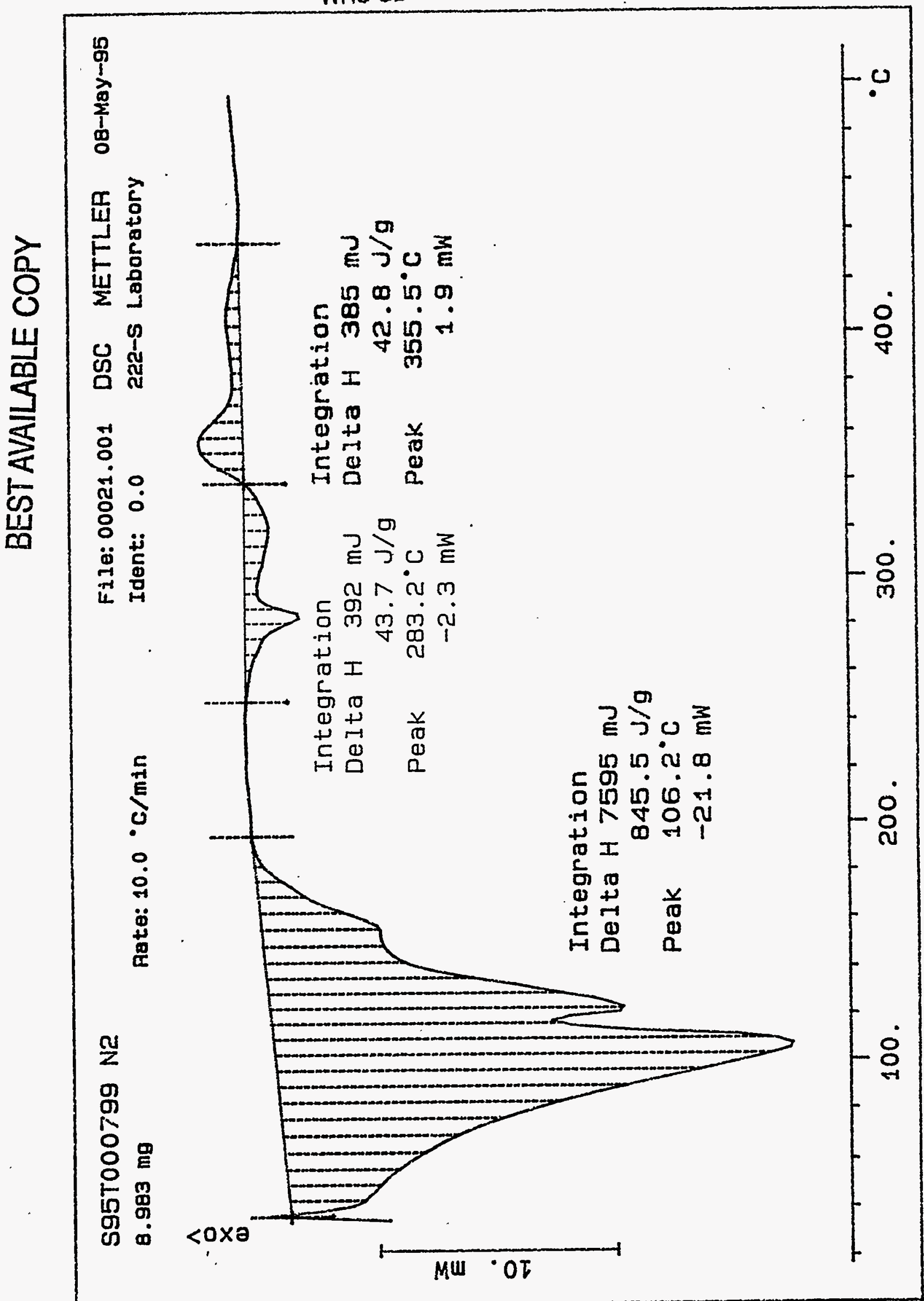


BEST AVAILABLE COPY

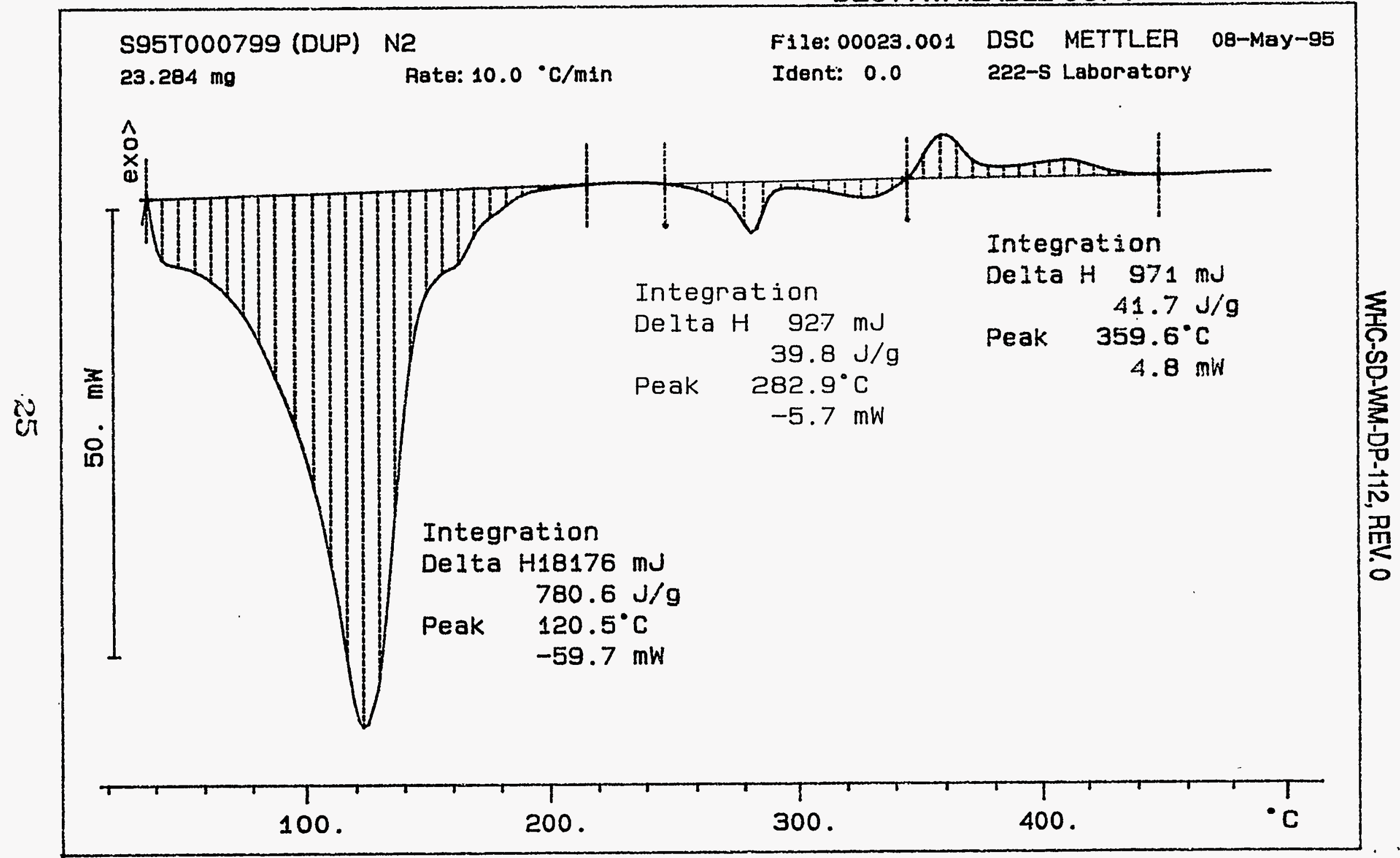




\section{BEST AVAILABLE COPY}

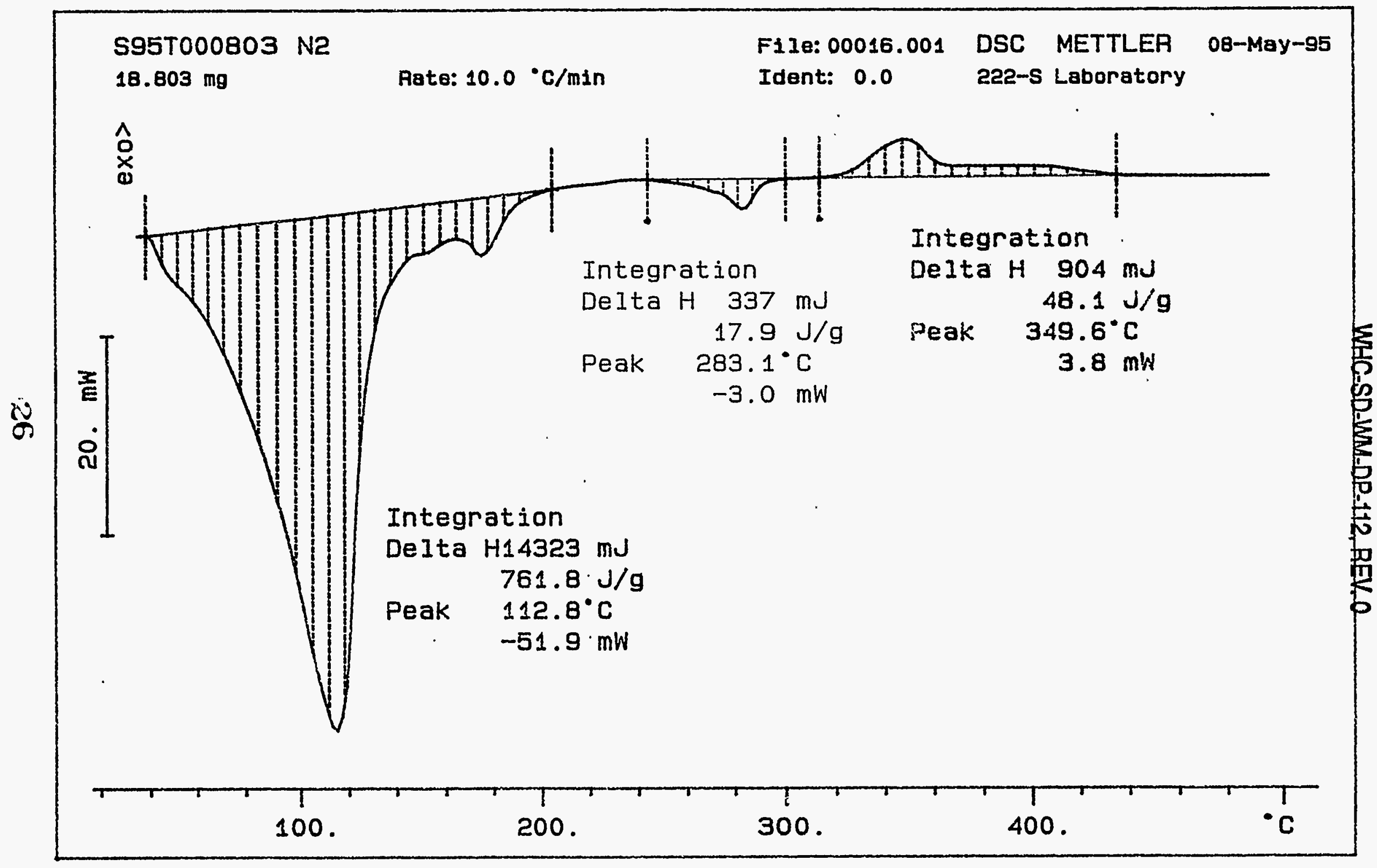


WHC-SD-WM-DP-112, REV. 0

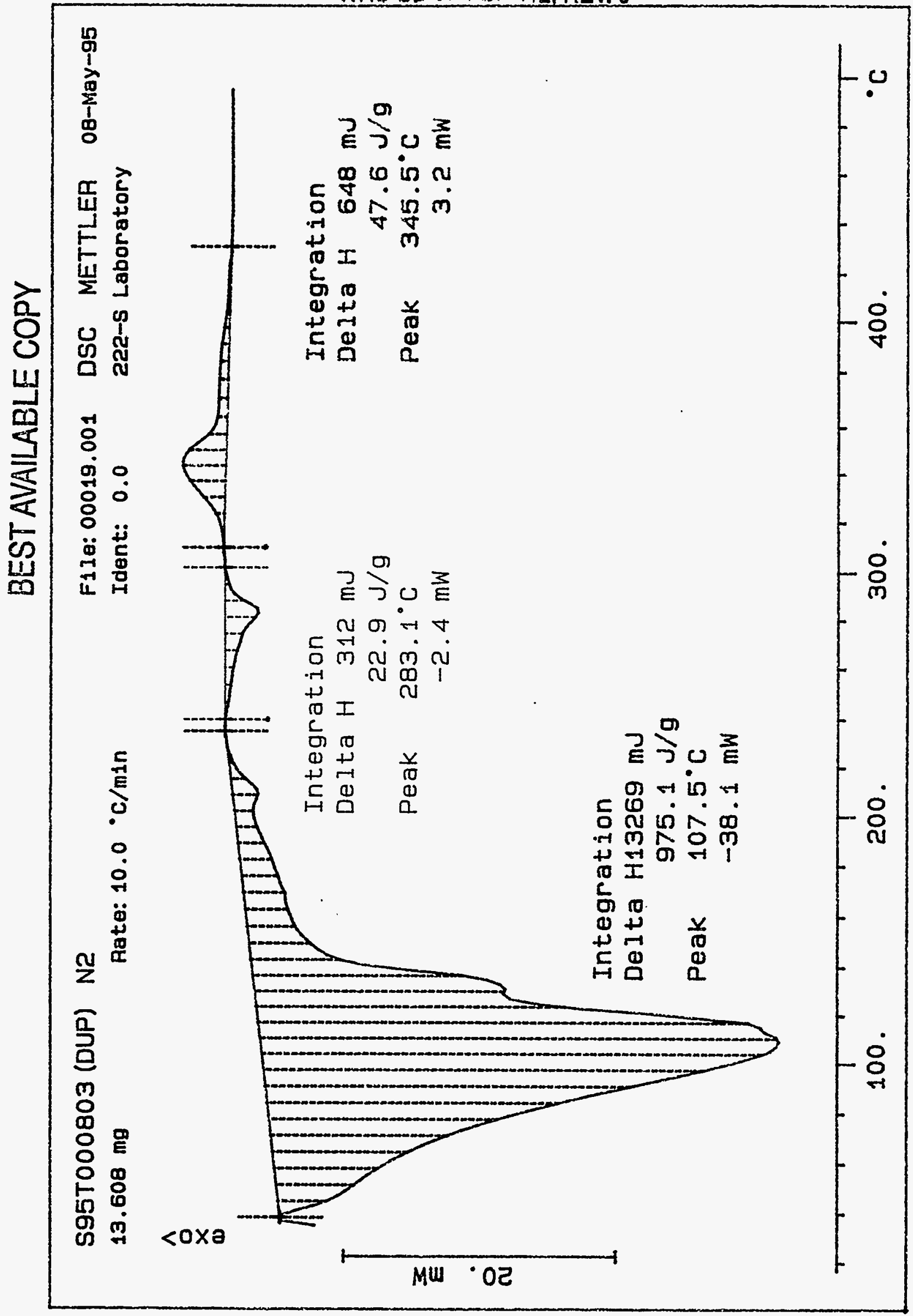


worklistrpt Version $2.002 / 21 / 95$

05/11/95 14:23

WHC-SD-WM-DP-112, REV. 0

Page: $\quad 1$

\section{LABCORE Data Entry Template for Worklist\# 1217}

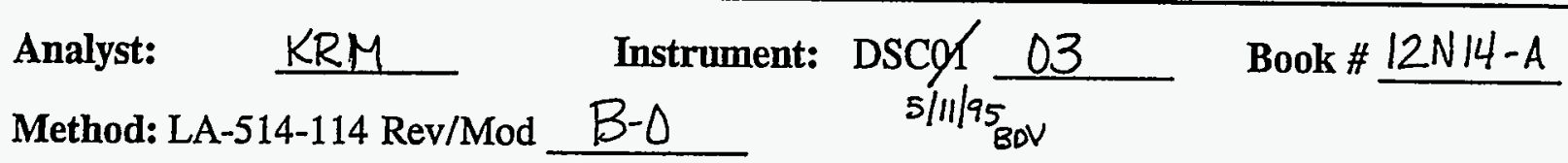

Worklist Comment: Please run C-203 DSC under N2. bdv

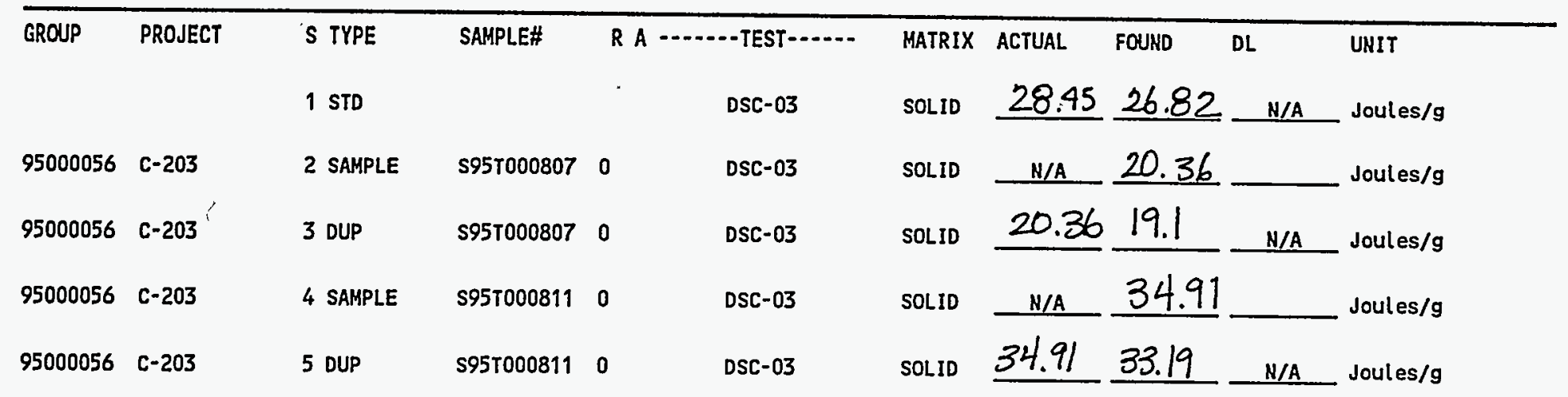

\section{Final page for worklist \# 1217}

$\frac{\text { See attached for dianatures } 5 / 11 / 95}{\text { Date }}$

Analyst Signature Date

S95T000807 produced two endotherms one at $105.7^{\circ} \mathrm{C}$ with a delta $\mathrm{H}$ of $807.9 \mathrm{~J} / \mathrm{g}$ and the second at $283.3{ }^{\circ} \mathrm{C}$ with a delta $H$ of $11.8 \mathrm{~J} / \mathrm{g}$.

Data Entry Comments: S957000811 produced two endotherms one at $117.1{ }^{\circ} \mathrm{C}$ with

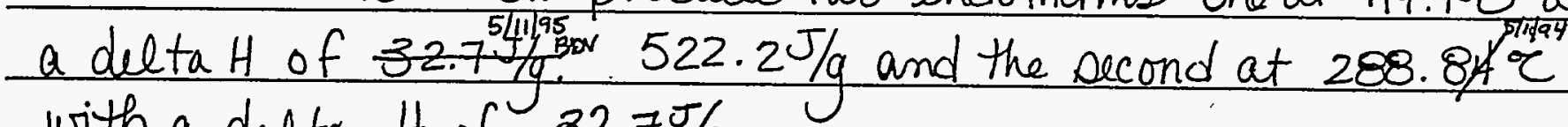
with a delta $H$ of $32.7 \%$.

Units shown for $Q C$ (SPK \& STD) may not reflect the actual units. $D L=$ Detection Limit, $S=$ Worklist Slot Number, $R=$ Replicate Number, $A=$ Aliquot Code. 
worklistrpt Version 2.0 02/21/95

05/04/95 14:42

WHC-SD-WMA-DP-112, REV. 0

\section{LABCORE Data Entry Template for Worklist\# 1217}

Analyst: $\quad h R$ Instrument: $\mathrm{DSC} 01$

Book \# $12 N 14 \mathrm{~A}$

Method: $A-514-113 \quad \angle A \quad 514-114 / B-0$

Worklist Comment: Please run C-203 DSC under N2. bdv

\begin{tabular}{|c|c|c|c|c|c|c|c|c|c|c|}
\hline \multirow[t]{2}{*}{ GROUP } & \multirow[t]{2}{*}{ PROJECT } & S TYPE & \multirow[t]{2}{*}{ SAMPLE\# } & \multicolumn{2}{|c|}{ R A $\cdots---$ TEST $\cdots$} & \multirow{2}{*}{$\begin{array}{l}\text { MATRIX } \\
\text { SOLID }\end{array}$} & \multirow[t]{2}{*}{ ACTUAL } & \multirow[t]{2}{*}{ FOUND } & \multirow[t]{2}{*}{$\overline{D L}$} & \multirow{2}{*}{$\begin{array}{l}\text { UHIT } \\
\text { Joules/g }\end{array}$} \\
\hline & & 1 STD & & & DSC-01 & & & & & \\
\hline 95000056 & $c-203$ & 2 SAMPLE & S95T000807 & 0 & DSC-01 & SOLID & $\mathrm{N} / \mathrm{A}$ & & & Joules/g \\
\hline 95000056 & $C-203$ & 3 DUP & S95T000807 & 0 & DSC-01 & SOLID & & & $\mathrm{N} / \mathrm{A}$ & Joules/g \\
\hline 95000056 & $C-203$ & 4 SAMPLE & S95T000811 & 0 & DSC-01 & SOLID & $\mathrm{N} / \mathrm{A}$ & & & Joules/g \\
\hline 95000056 & $C-203$ & 5 DUP & S95T000811 & 0 & DSC-01 & SOLID & & & $\mathrm{N} / \mathrm{A}$ & Joules/g \\
\hline
\end{tabular}

\section{Final page for worklist \# 1217}

Analyst Signature Date $5-8-95$

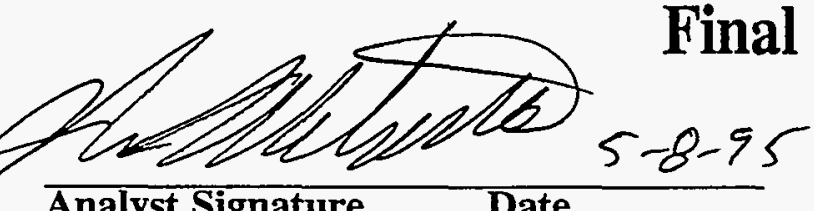

Analyst Signature Date

Data Entry Comments:

Other instrument was used.

Units shown for $Q C$ (SPK \& STD) may not reflect the actual units. $D L=$ Detection Limit, $S=$ Worklist Slot Number, $R=$ Replicate Number, $A=$ Aliquot Code. 
Curve 1: DSC

File Info: Ind050801 Mon May 8 16: $38: 151995$

BEST AVAILABLE COPY

Sample Weight: $6.687 \mathrm{mg}$

Indium at 10c/min SIGNATURE BELOW REPRESENIS CHEMICAL TECHNOLOGIST/CHEMIST THAT

$12 \mathrm{~N} 14-A_{5 / 9 / 95}$ BOV COMPLETED/VERIFIED THE CALIBRATION/ANALYSIS ON PAGES 30 TO 34.

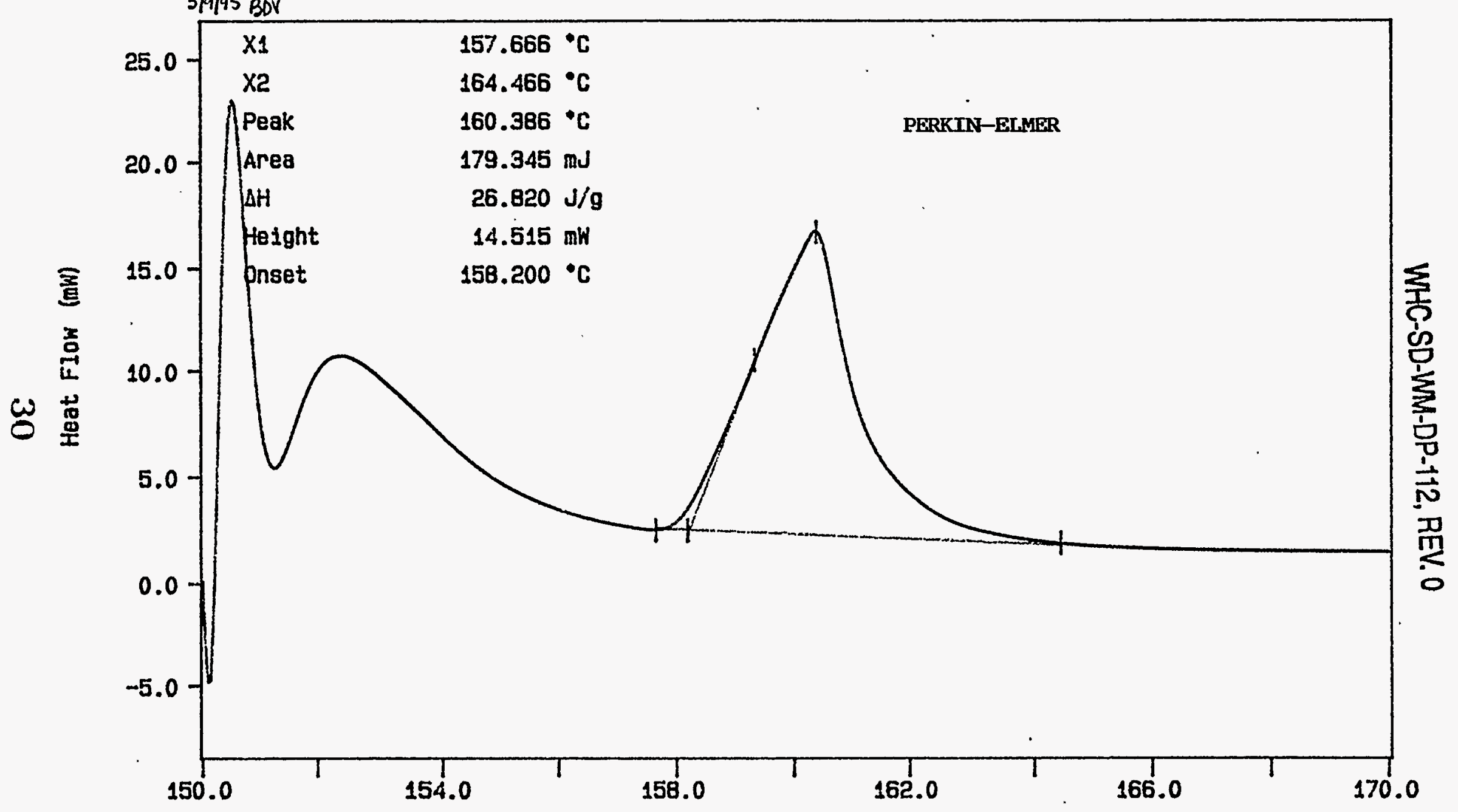

Ne exotherm down TEMp: $150: 8$ \& TIHE1: Temperature ('C) KR Monteith Hestinghouse Hanford Co. Mon May B 16: 58: 191995

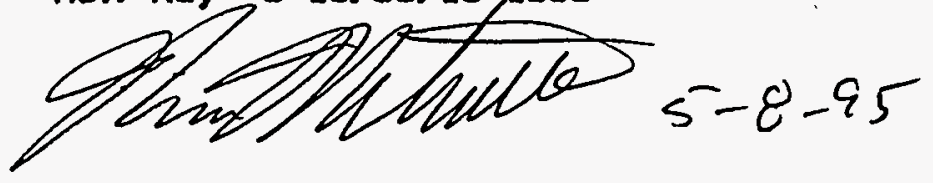


Curve 1: DSC

File info: sam050801 Mon May 8 18:08: 241995

Sample Weight: $19.280 \mathrm{mg}$

BEST AVAILABLE COPY

s95T000807. $10 \mathrm{C} / \mathrm{min}$

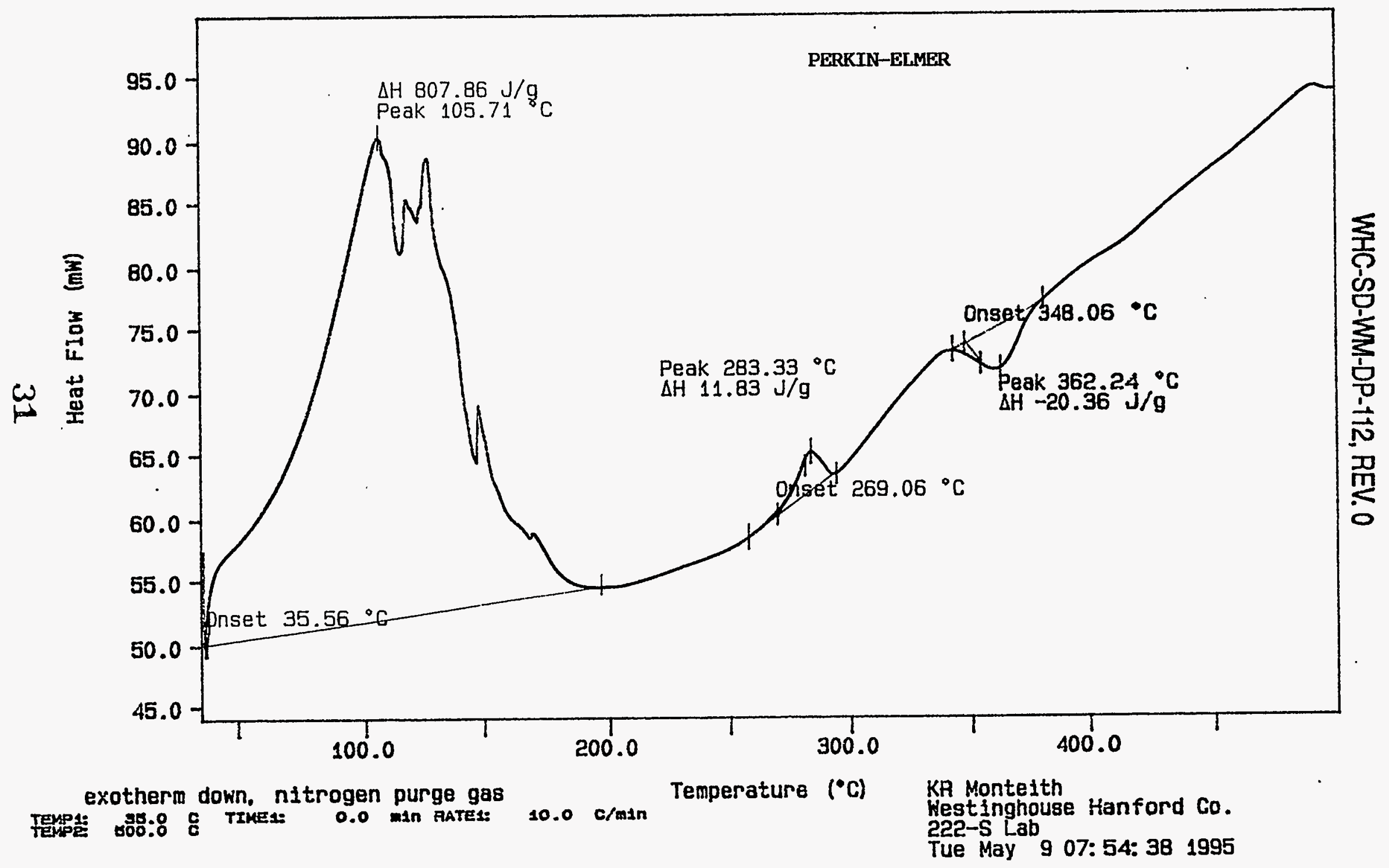




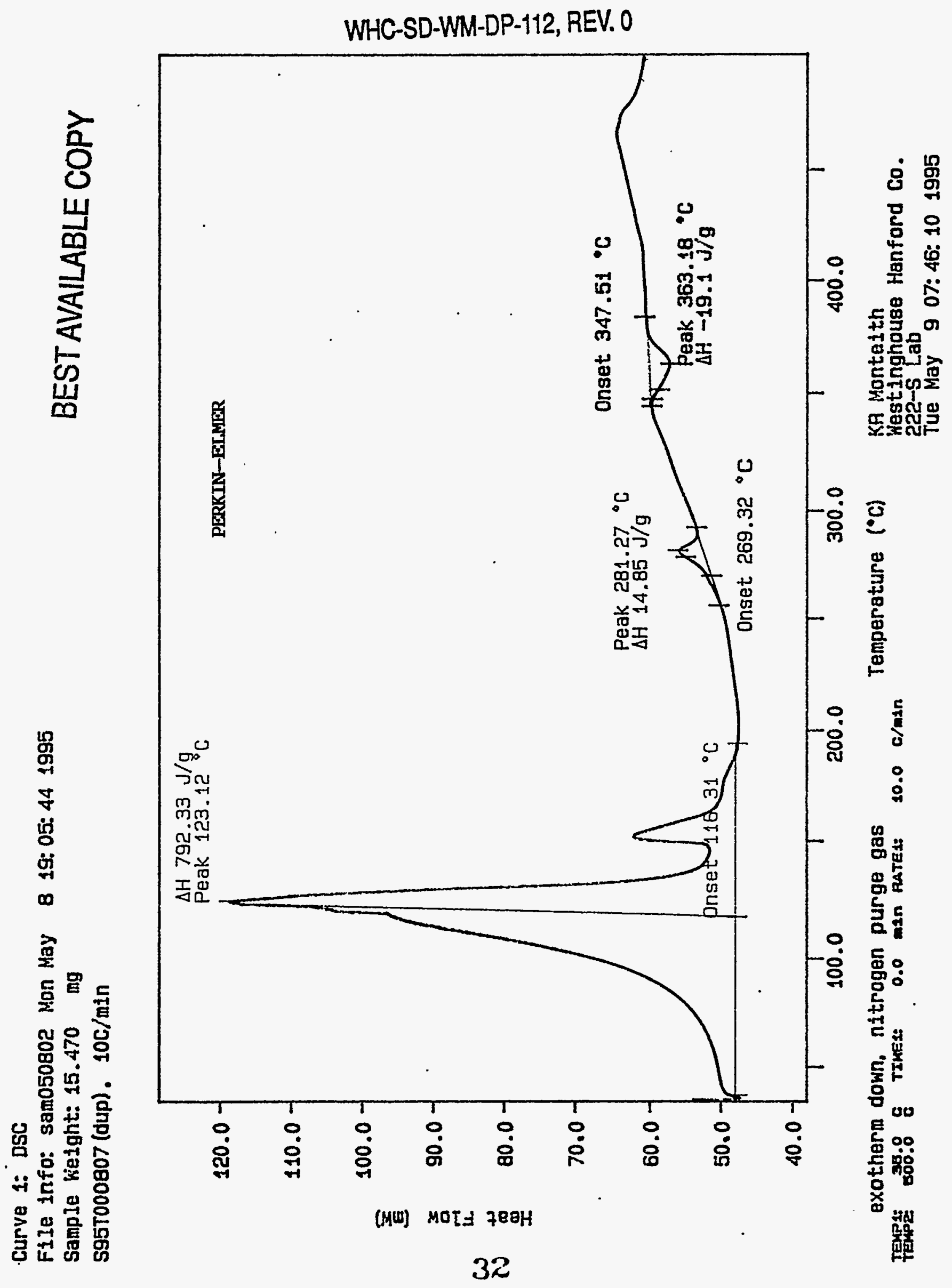


Curve 1: DSC

File info: sam050803 Mon May 8 20:19:51 1995

Sample KeIght: $15.819 \mathrm{mg}$

595T000811, 10C/min

\section{BEST AVAILABLE COPY}

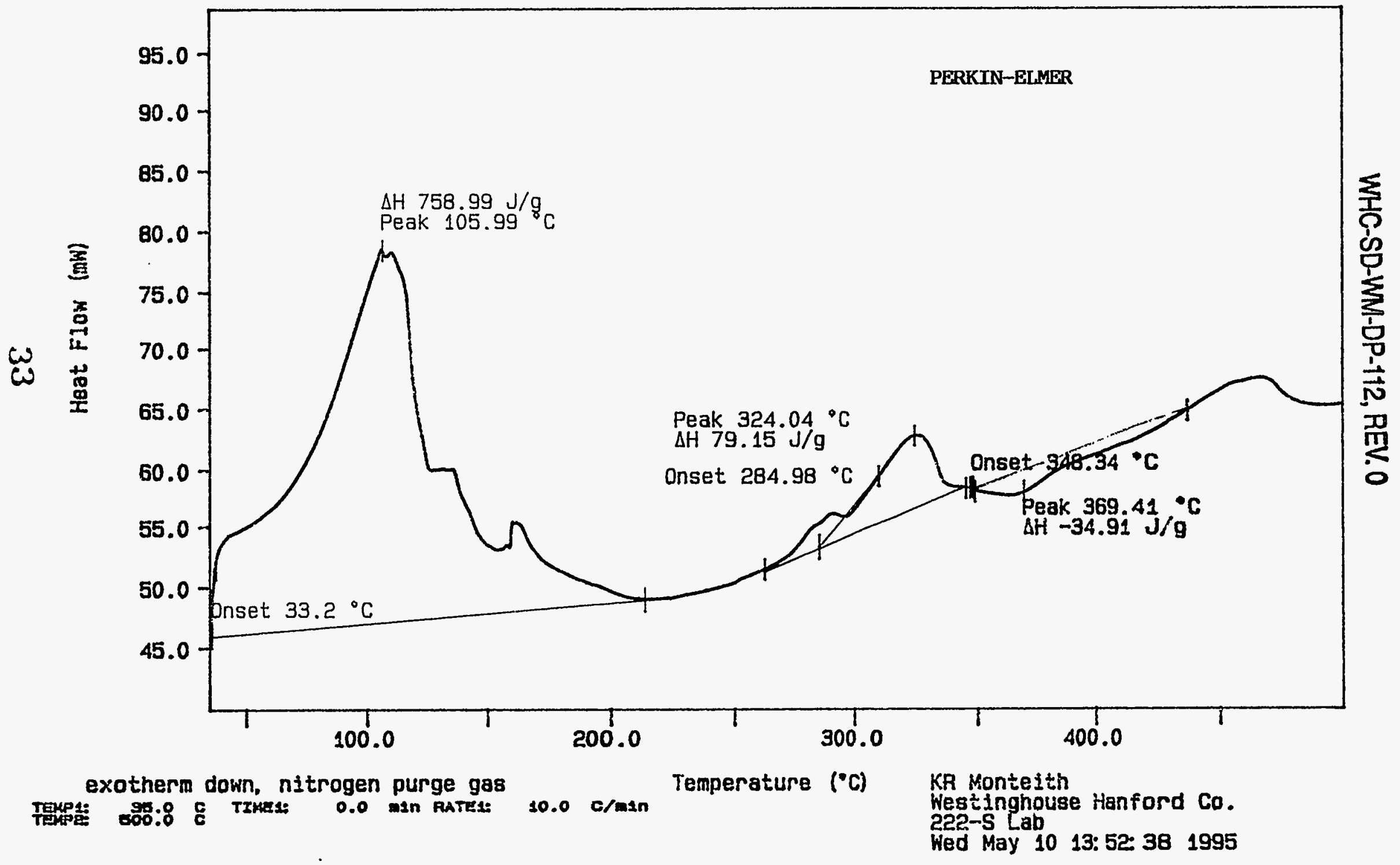


Curve 1: DSC

File info: 8am050804 Mon May 8 22 10: 411995

Sample Hejght: $20.110 \mathrm{mg}$

s95T0008:1 (dup). 10c/mśn

\section{BEST AVAILABLE COPY}

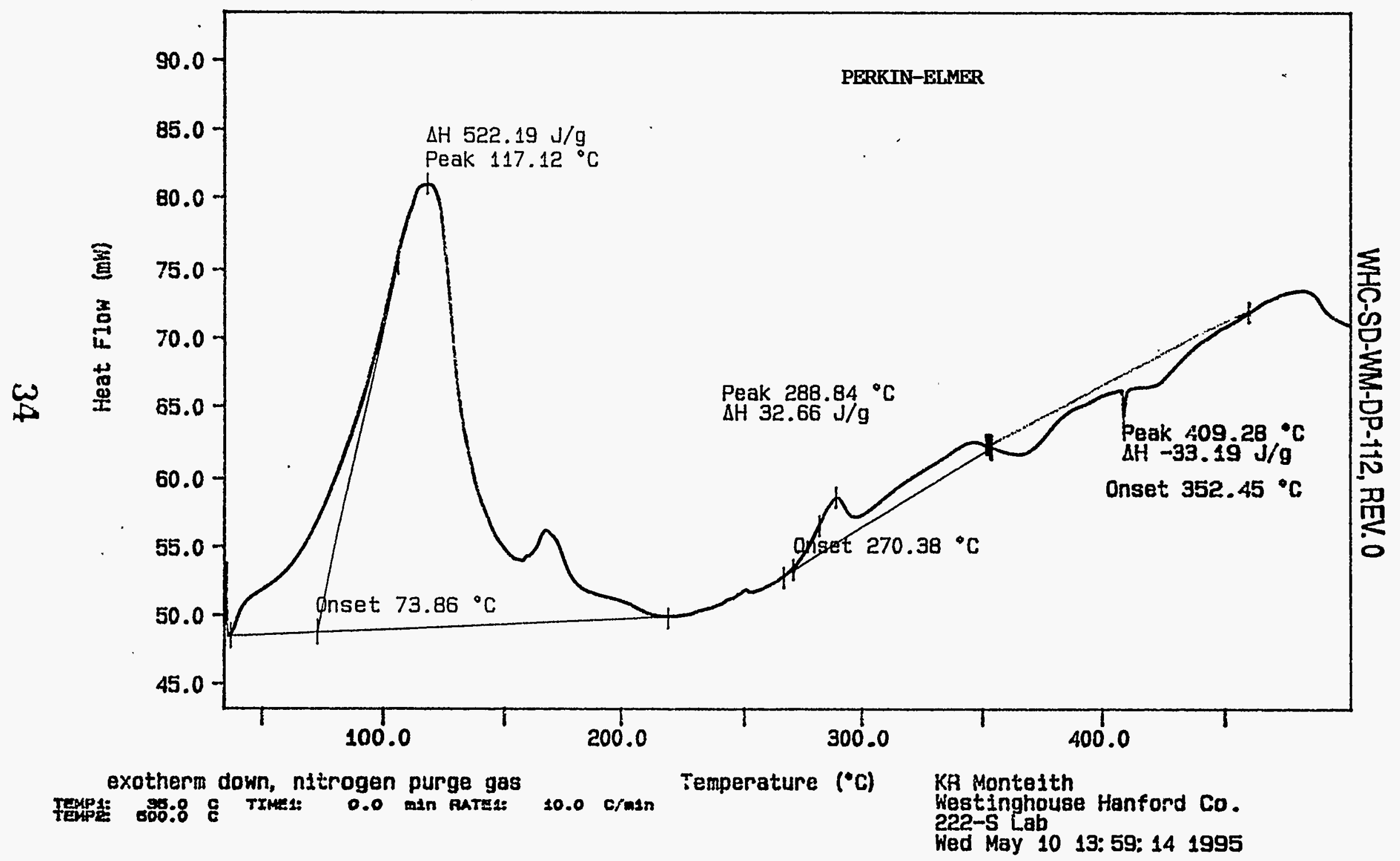




\begin{tabular}{|c|c|c|c|}
\hline \multicolumn{4}{|c|}{$C-203$} \\
\hline \multicolumn{4}{|c|}{ CALCULATED DRY DSC } \\
\hline SAMPLE NO. & DSC RESULT $(J / g)$ & TGA RESULT (\% water) & DRY DSC RESULT \\
\hline$\$ 95 T 000799$ & 42.8 & 35.17 & 66.02 \\
\hline $799 D$ & 41.7 & 36.17 & 64.32 \\
\hline 803 & 48.1 & 50.58 & 97.33 \\
\hline 803D & 47.6 & 50.58 & 96.32 \\
\hline 807 & 20.36 & 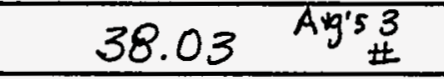 & 32.85 \\
\hline 807D & 19.1 & $38.03 \quad$ Avgs 3 & 30.82 \\
\hline 811 & 34.91 & 31.38 & 50.87 \\
\hline $811 D$ & 33.19 & 31.38 & 48.37 \\
\hline & . & & \\
\hline & & & \\
\hline & & & \\
\hline & & & \\
\hline & & . & \\
\hline & & & \\
\hline & & & \\
\hline & & $\bar{r}$ & \\
\hline & & & \\
\hline & & & \\
\hline & & & \\
\hline & & & \\
\hline & & & \\
\hline & & & \\
\hline & & & \\
\hline
\end{tabular}




Analyst: $\quad K R M \quad$ Instrument: TGA01_ Book\# $42 \times 8 A$

Method: LA-560-112 Rev/Mod A-2

Worklist Comment: Please run C-203 TGA under N2. bdv

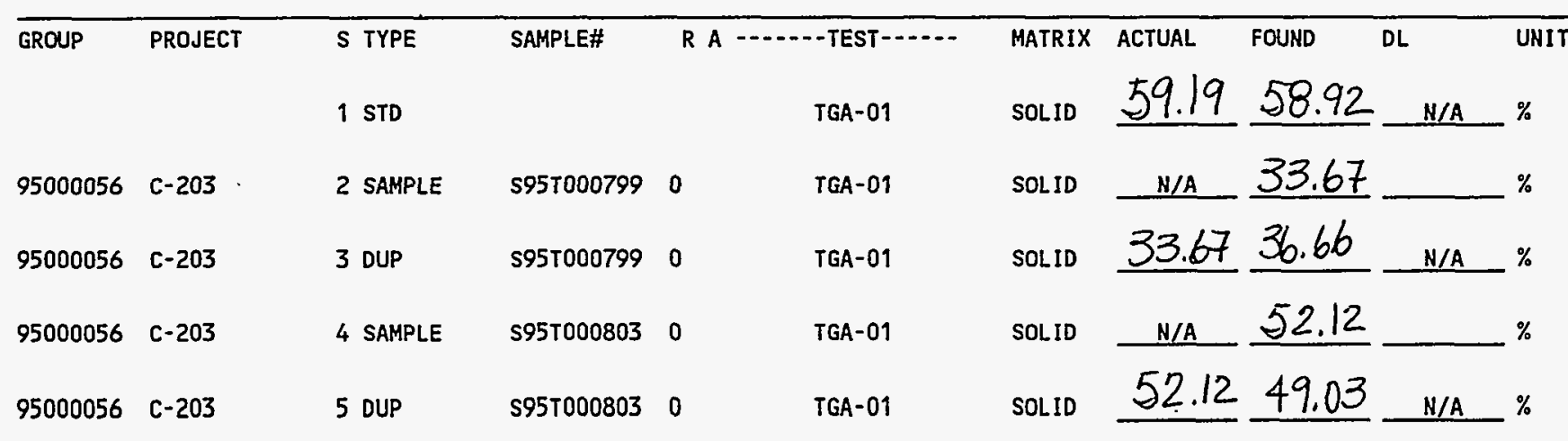

Final page for worklist \# 1237

Analyst Signature Date

Verified by Blandina Valenzuela 5/9/95

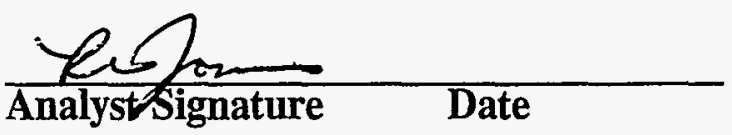

Data Entry Comments: 9957000799 produced another weight loss step of $10.31 \%$ at 327.0 $\$ 957000803$ procluced a second weight loss step of $6.91 \%$ at $331.0^{\circ} \mathrm{C}$ 
SIGNATURE BELOW REPRESENIS CHEMICAL TECHNOLOGIST/CHEMIST THAT COMPLETED/VERIFIED THE CALIBRATION/ANALYSIS ON PAGES 38 TO

\section{BEST AVAILABIE COPY}

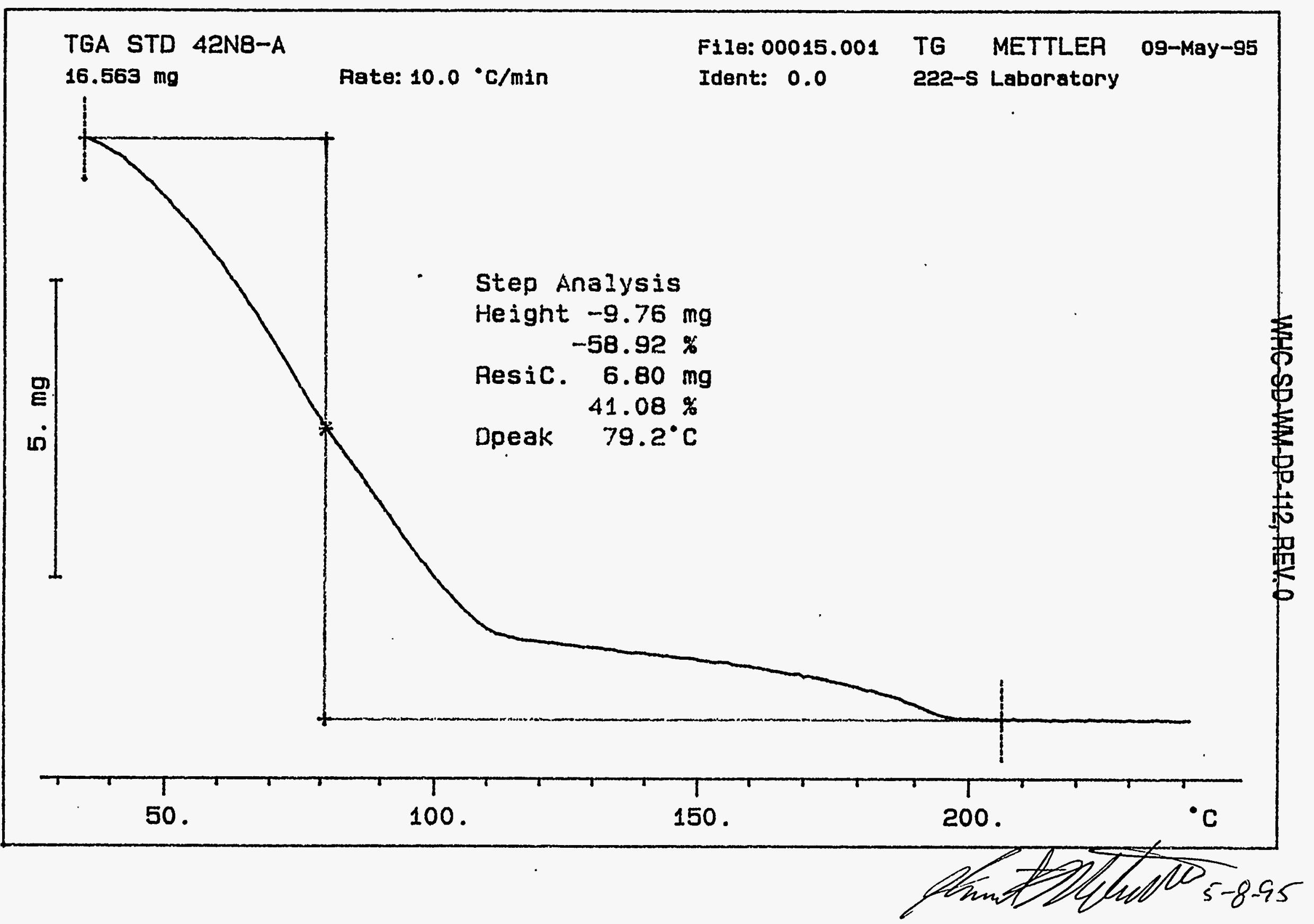




\section{BEST AVAILABLE COPY}

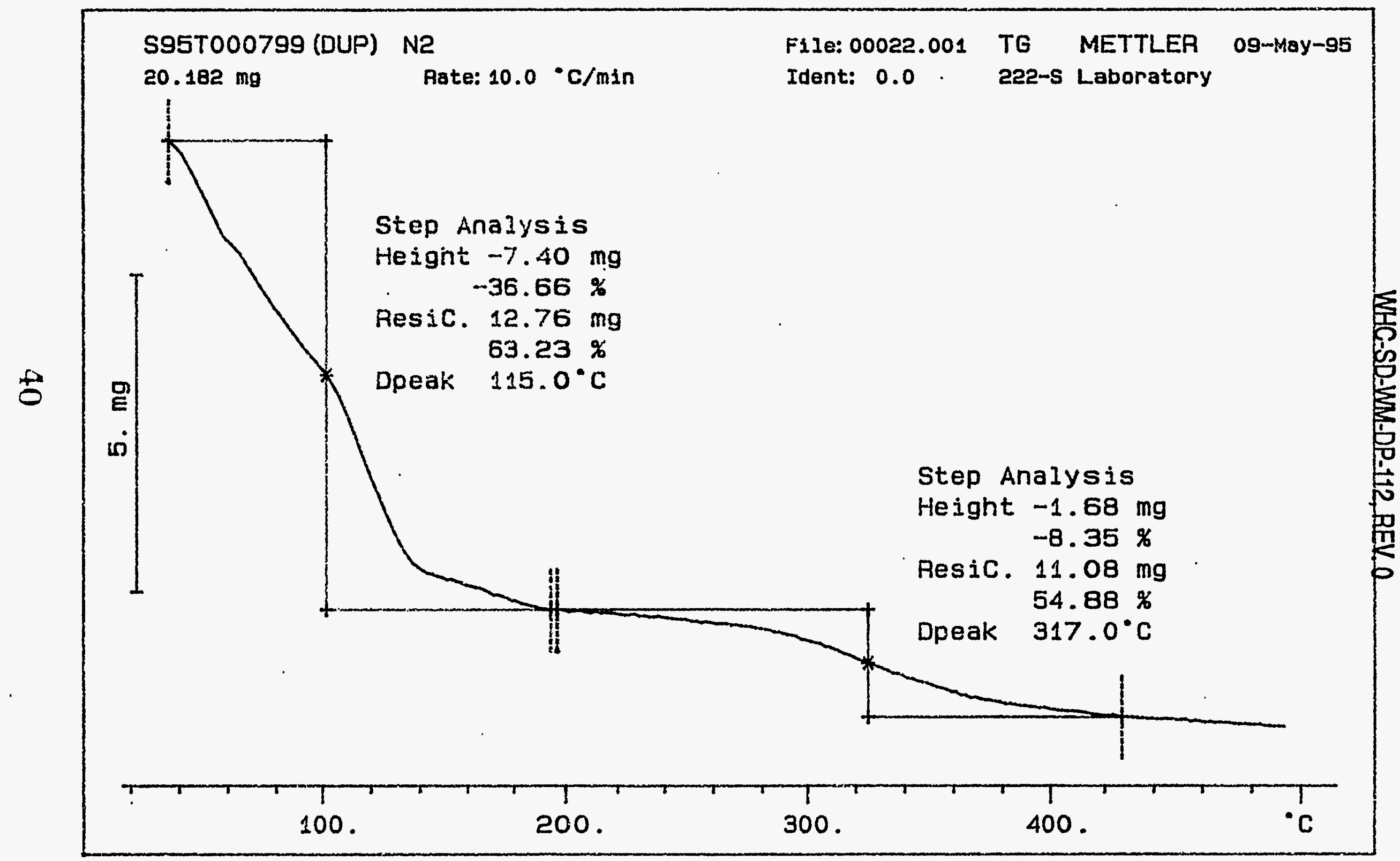


WHC-SD-WM-DP-112,REV.O

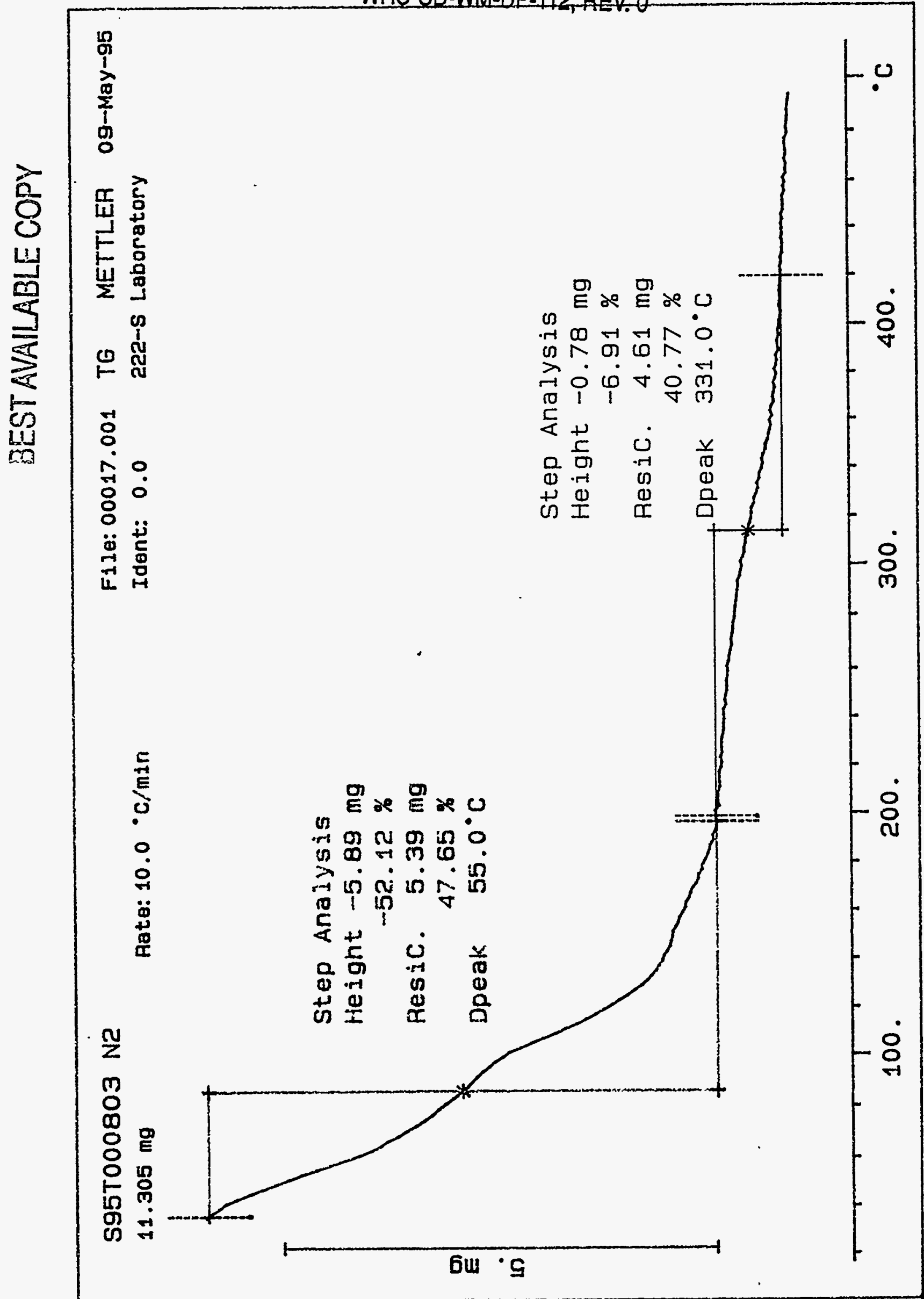




\section{ZEST AVALABLE COPY}

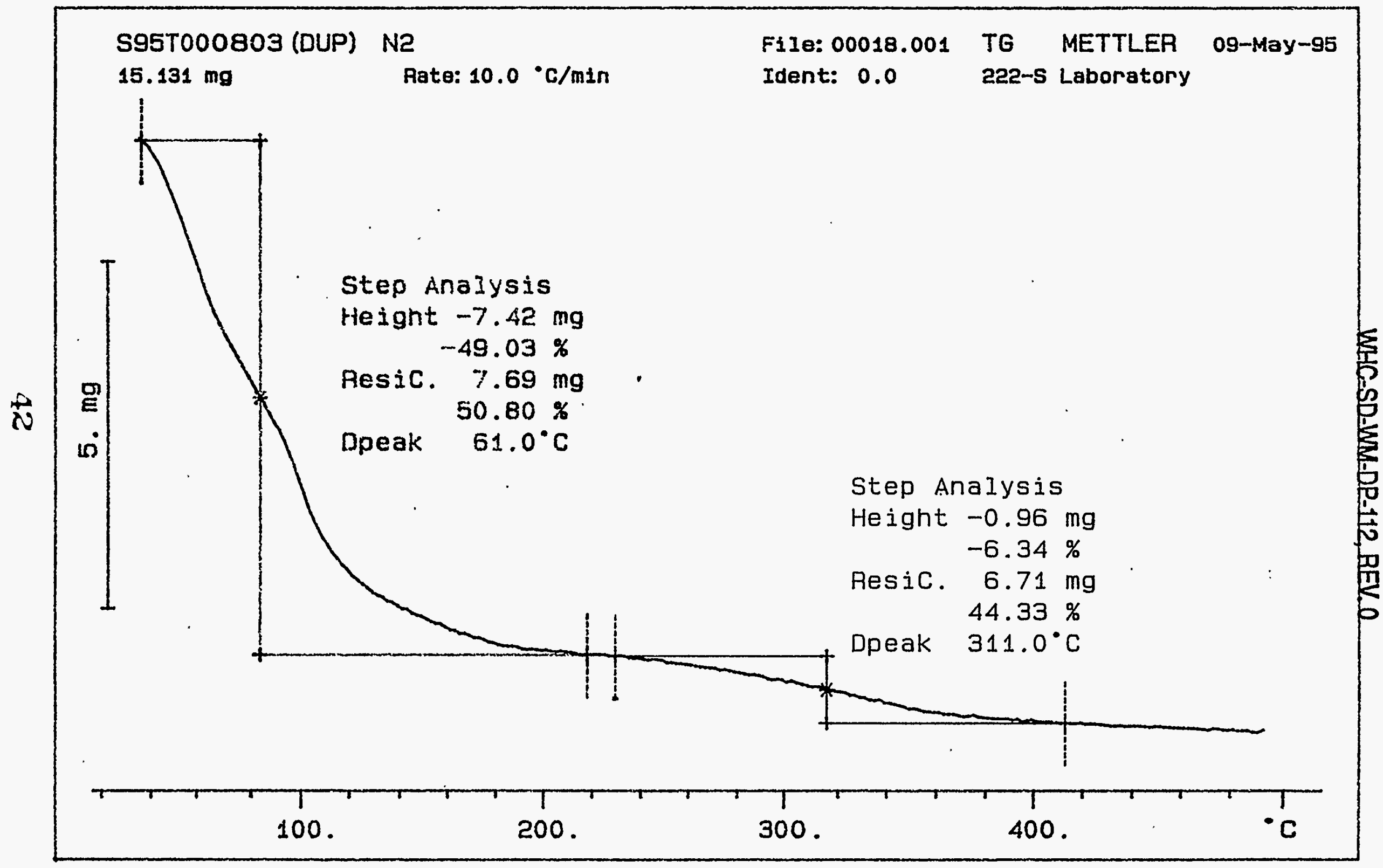


worklistrpt Version 2.0 02/21/95

WHC-SD-WM-DP-112, REV. 0

Page: 1

05/10/95 06:54

\section{LABCORE Data Entry Template for Worklist\# 1238}

\begin{tabular}{|c|c|c|c|}
\hline Analyst: & KRM & Instrument: TGAØ1 03 & Book \# $42 N 8-A$ \\
\hline
\end{tabular}

Worklist Comment: Please run C-203 TGA under N2. bdv

\begin{tabular}{|c|c|c|c|c|c|c|c|c|c|c|}
\hline GROUP & PROJECT & $S$ TYPE' & SAMPLE\# & & -TEST...- & MATRIX & ACTUAL & FOUND & & UNIT \\
\hline & & 1 STD & & & TGA-03 & SOLID & 59.19 & 57.39 & $N / A$ & $\%$ \\
\hline 95000056 & $c-203$ & 2 SAMPLE & S95T000807 & 0 & TGA-03 & SOLID & N/A & 41.04 & & $\%$ \\
\hline 95000056 & $c-203$ & 3 DUP & 595T000807 & 0 & TGA-03 & SOLID & 41.04 & 33.26 & $\mathrm{~N} / \mathrm{A}$ & $\%$ \\
\hline & & 4 STD & & & TGA-03 & SOLID & 59.19 & 57.68 & N/A & $\%$ \\
\hline 95000056 & $c-203$ & 5 TRIPL & S95T000807 & 0 & TEA-03 & SOLID & 41.04 & 39.79 & N/A & $\%$ \\
\hline 95000056 & $c-203$ & 6 SAMPLE & S95T000811 & 0 & TGA-03 & SOLID & $\mathrm{N} / \mathrm{A}$ & 30.98 & . & $\%$ \\
\hline 95000056 & $c-203$ & 7 DUP & s95T000811 & 0 & TGA-03 & SOLID & 30.98 & 31.78 & N/A & $\%$ \\
\hline
\end{tabular}

Final page for worklist \# 1238

Su attached for other signatures 5/10/95

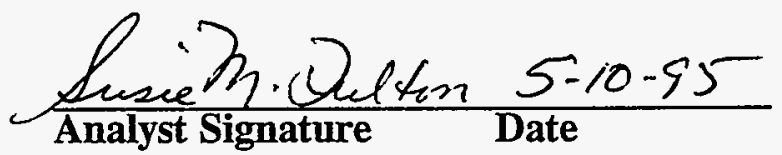

Verified by Blandina Valenzula $5 / 10 / 95$
$5 / 11 / 95$
BOV

\$95T000807 produced a Decond weight loss step of $5.95 \%$ at a pproximately. $310^{\circ} \mathrm{C}$. The triplicate for this cample was analyzed a couple of days after initial analyois, once chemist was able to look at the data. 5957000811 produced a second weight loss step of $5.72 \%$ at approximalely $310^{\circ} \mathrm{C}$

Data Entry Comments:

595000807 - Dark Brown, Thick, creamy material

S95000 811 - DarkBrown, thick, stiff matercil

Units shown for QC (SPK \& STD) may not reflect the actual units. $D L=$ Detection Limit, $S=$ Worklist Slot Number,

$R=$ Replicate Number, $A=$ Aliquot Code. 


\section{LABCORE Data Entry Template for Worklist\# 1238}

Analyst: $\quad K R M \quad$ Instrument: TGA01_ Book \# $4 / 2 N 8 A$

Method: $\perp A=560-112 \operatorname{Red} \angle A 5 / 4-114 / B-0$

Worklist Comment: Please run C-203 TGA under N2. bdv

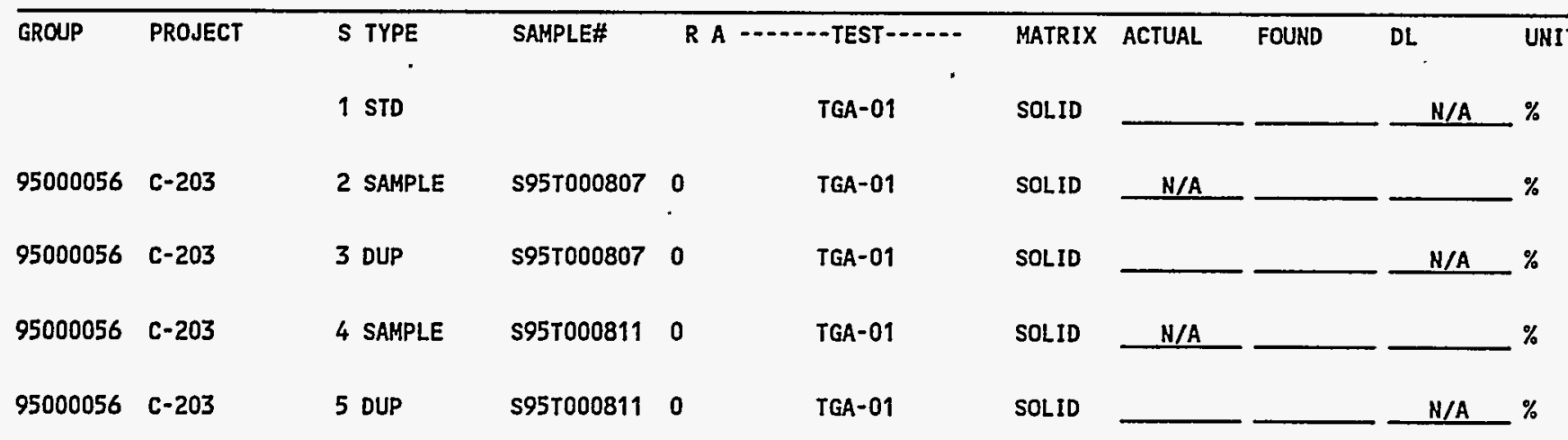

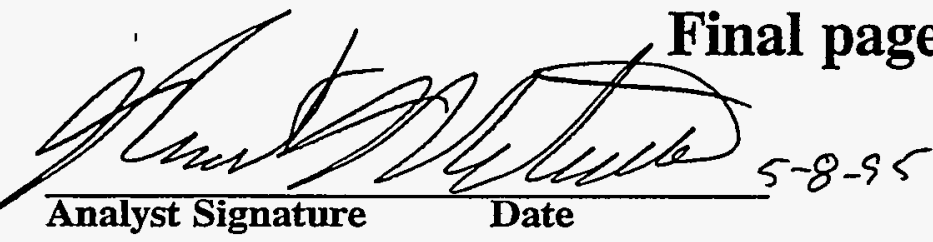

Analyst Signature Date

Data Entry Comments:

Other instrument was used.

Units shown for QC (SPK \& STD) may not reflect the actual units. $D L=$ Detection Limit, $S=$ Worklist Slot Number, $R=$ Replicate Number, $A=$ Aliquot Code. 
Curve 1: TGA

File info: ter050801 Mon May 8 16:54: 451995

Sample Weight: 16.157

mg

\section{BEST AVAILABLE COPY}

42NBA Terlía

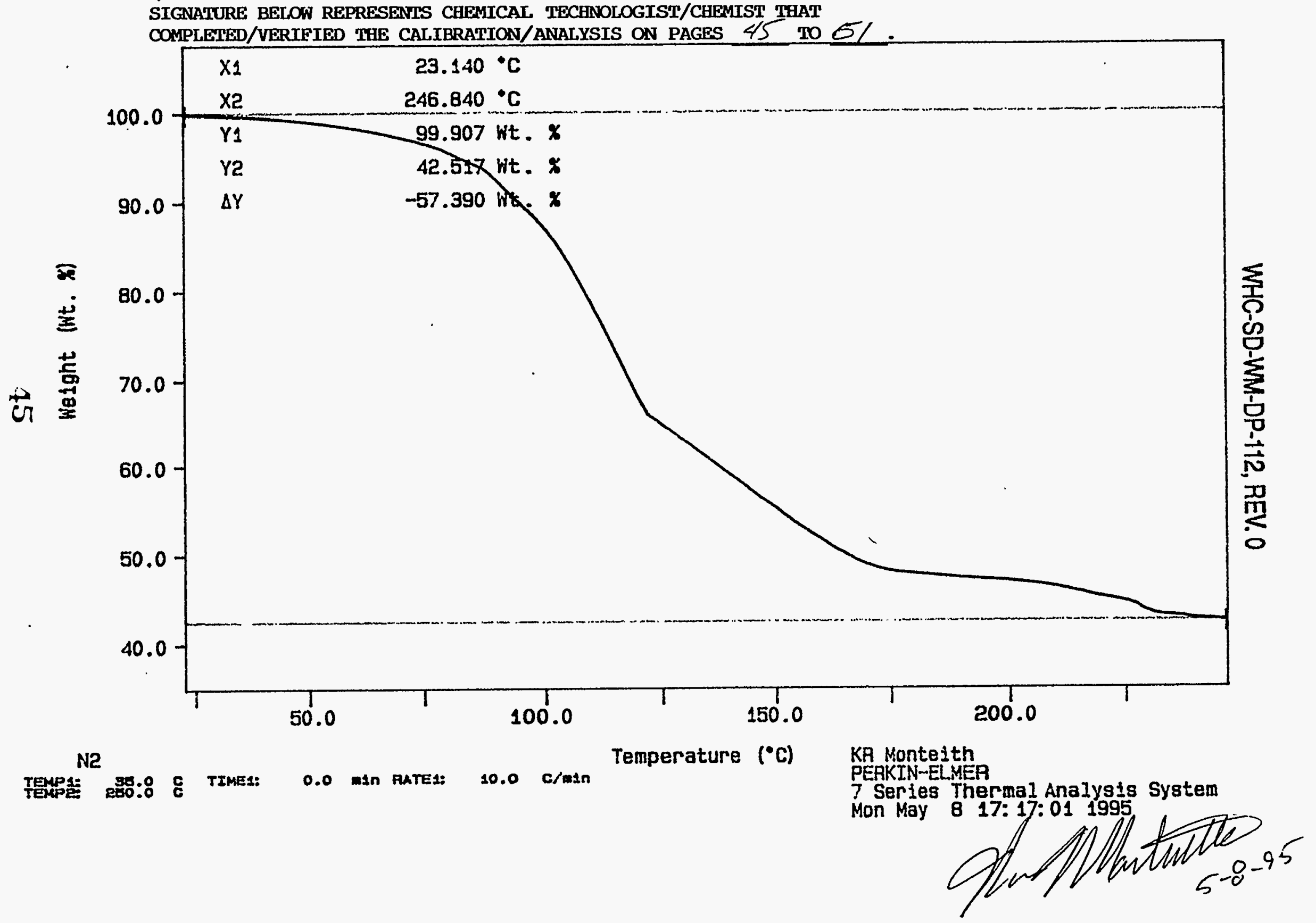


Curve 1: TGA

File Info: sam050801 Mon May 8 18: 11: 481995

Sample Kejght: 14.481 . mg

595T000870 fotif. 10C/min

BEST AVAILABLE COPY

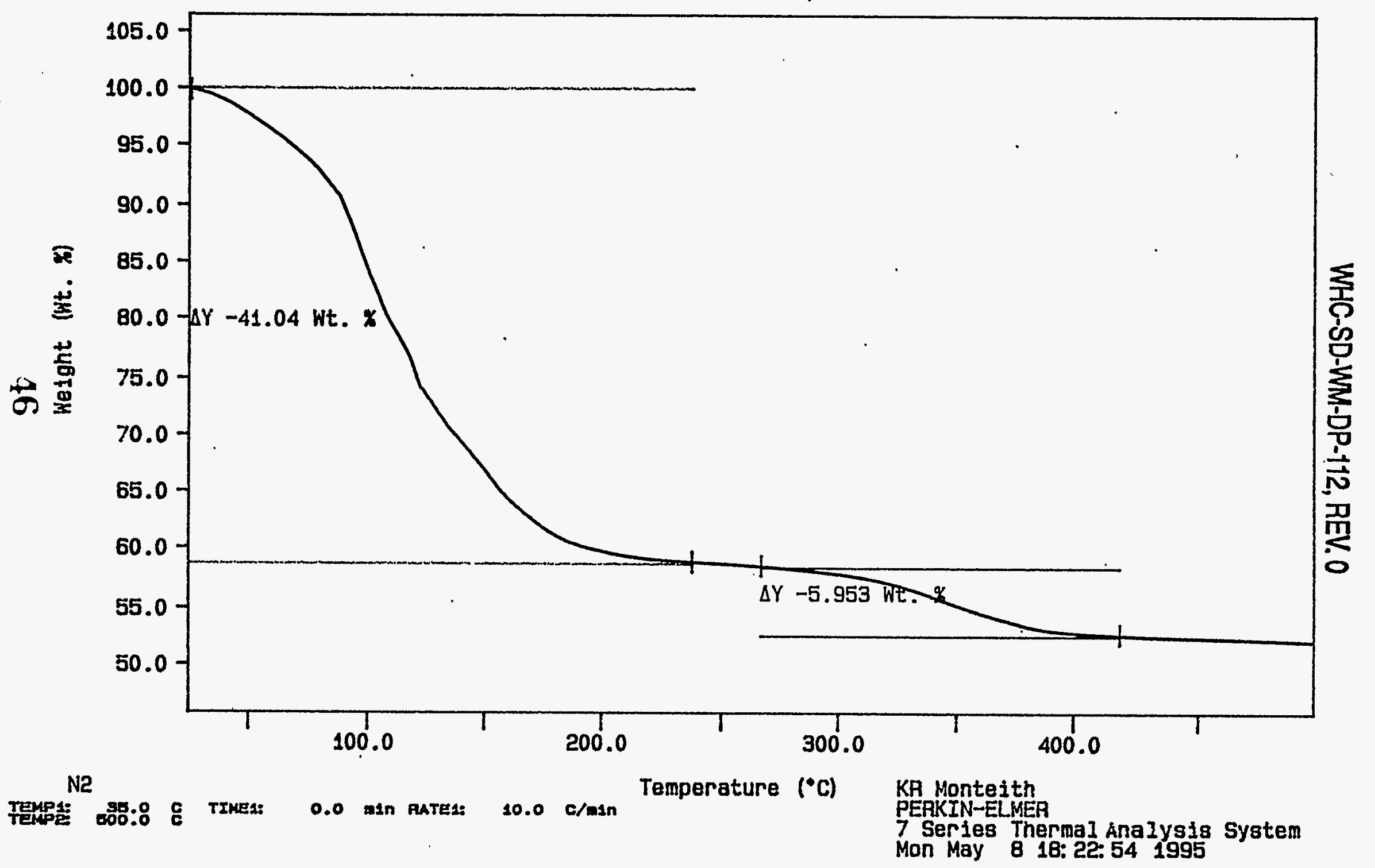




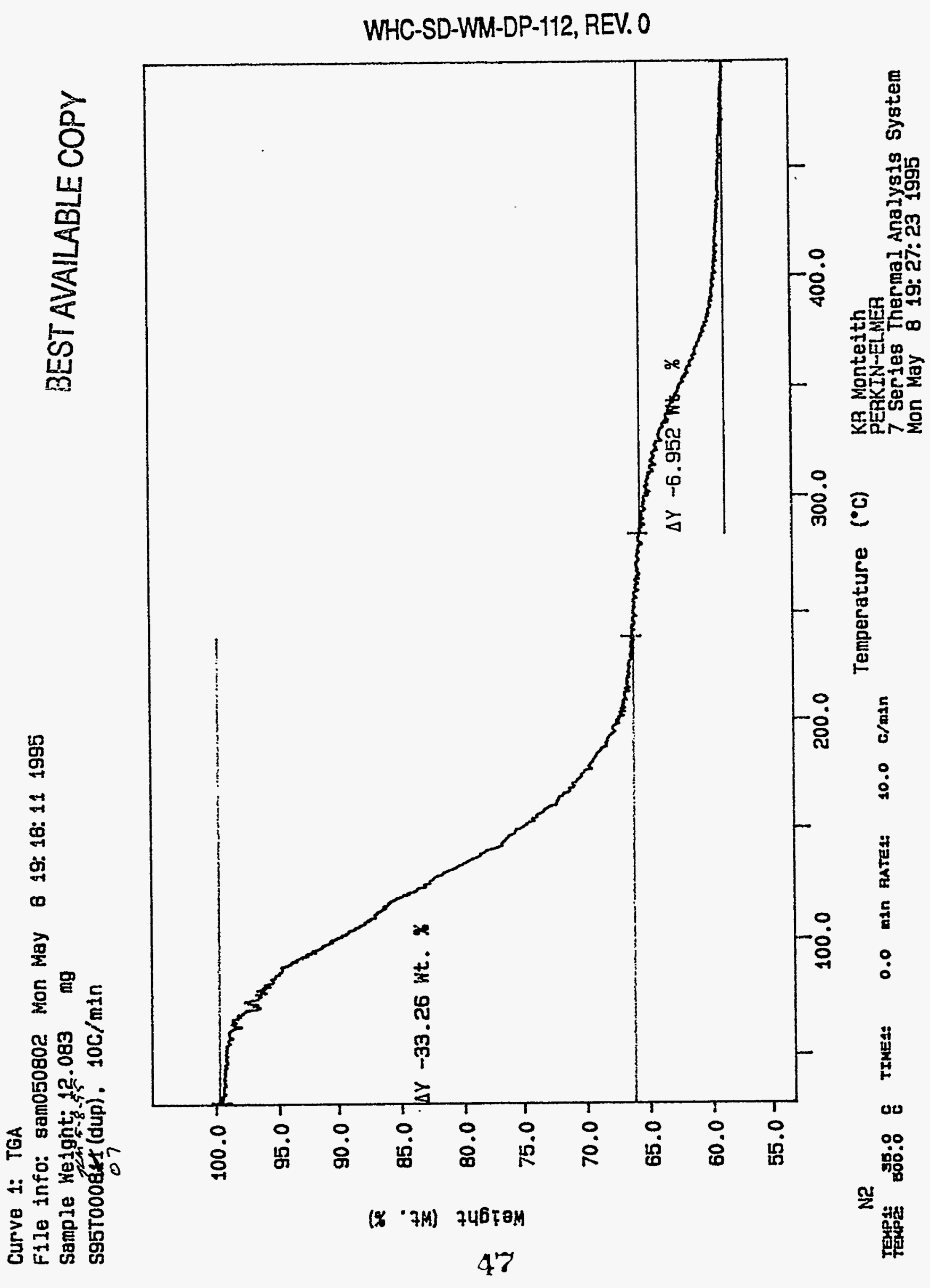




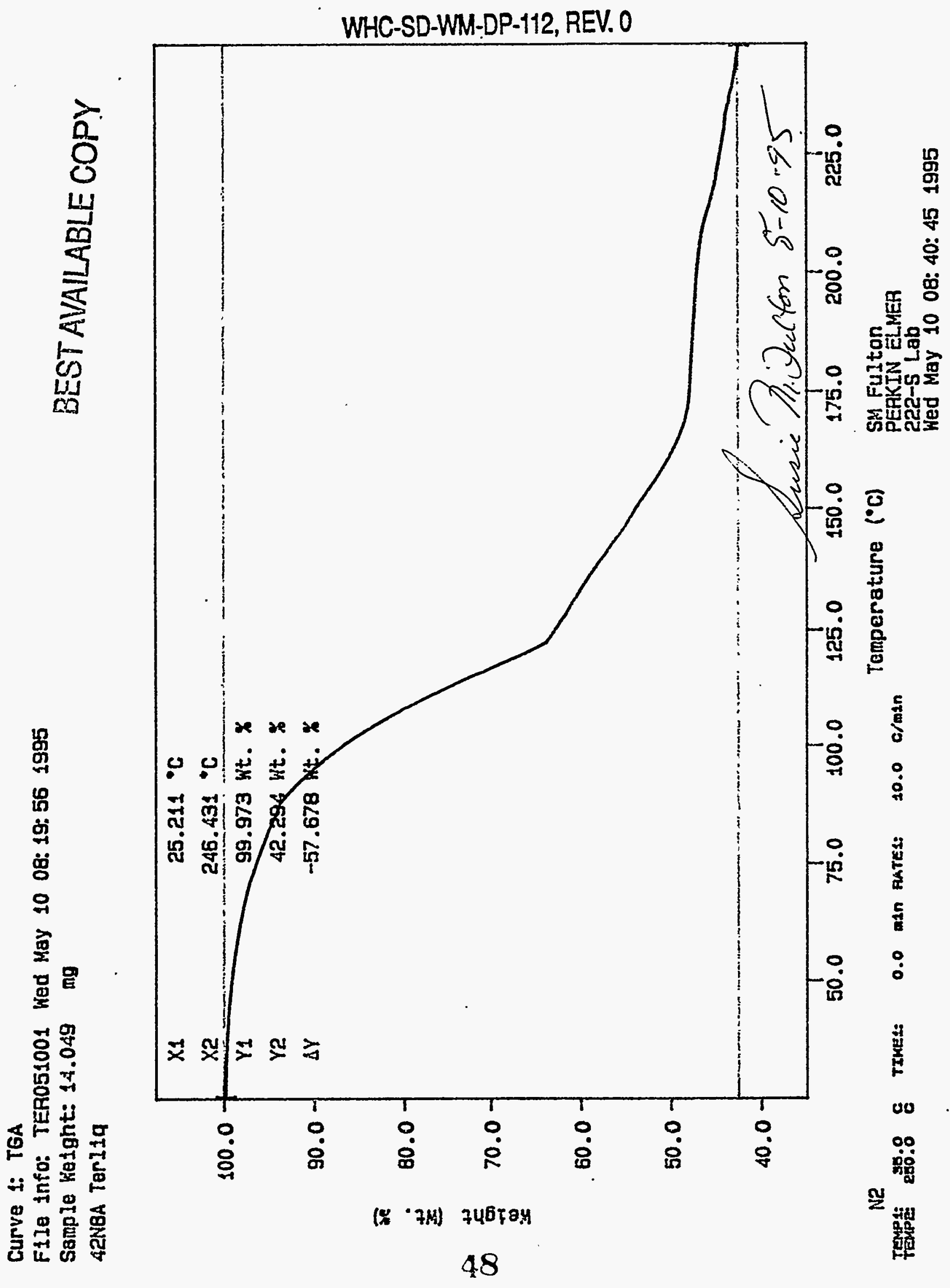


Curve 1: TGA

File info: SAPt051001 Ked May 10 09: 48: 391995

Sample Weight: $11.901 \mathrm{mg}$

s95T000807 (TAIPL). 10C/min

\section{BEST AVAILABLE COPY}

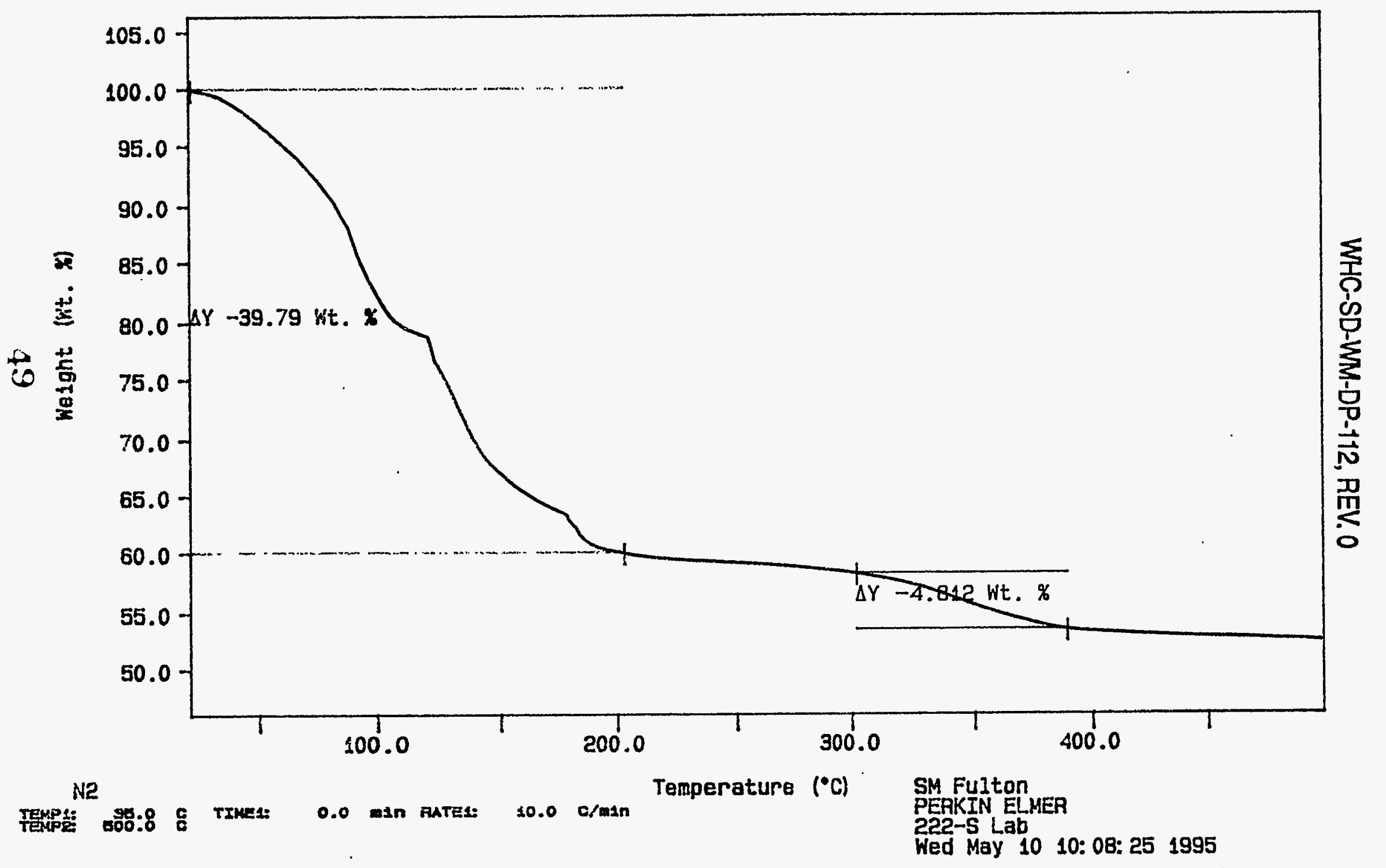


Curve 1: TGA

File infa: sam050803 Mon May 8 20: 25: 351995

Sample Weight: 14.450

$\mathrm{mg}$

\section{BEST AVAILABLE COPY}

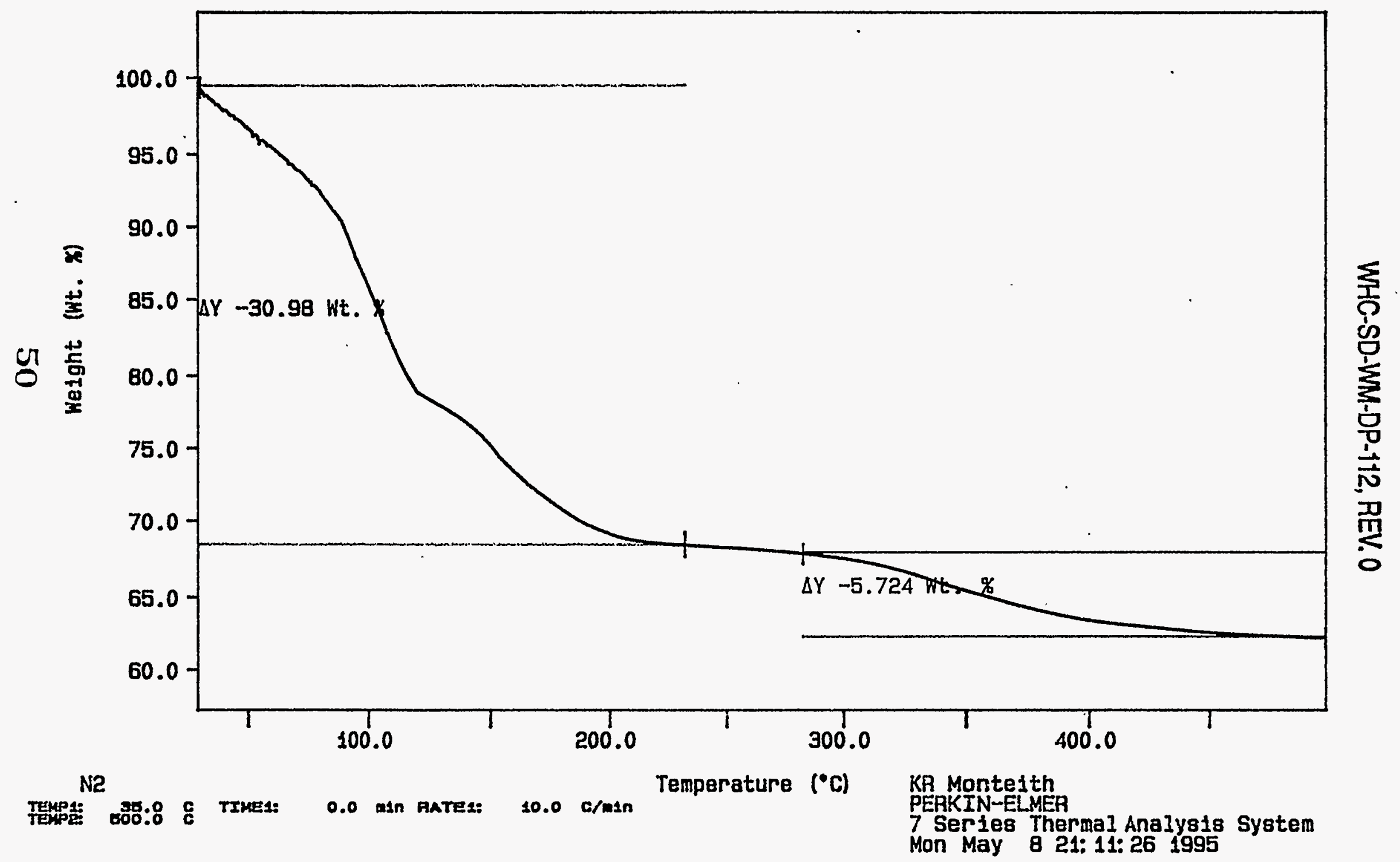


Curve 1: TGA

File Info: 8am050804 Mon May 8 22: 08: 151995

Sample Weight: $15.985 \mathrm{mg}$

595T000811 (dup). 10c/min

BEST AVAILABLE COPY

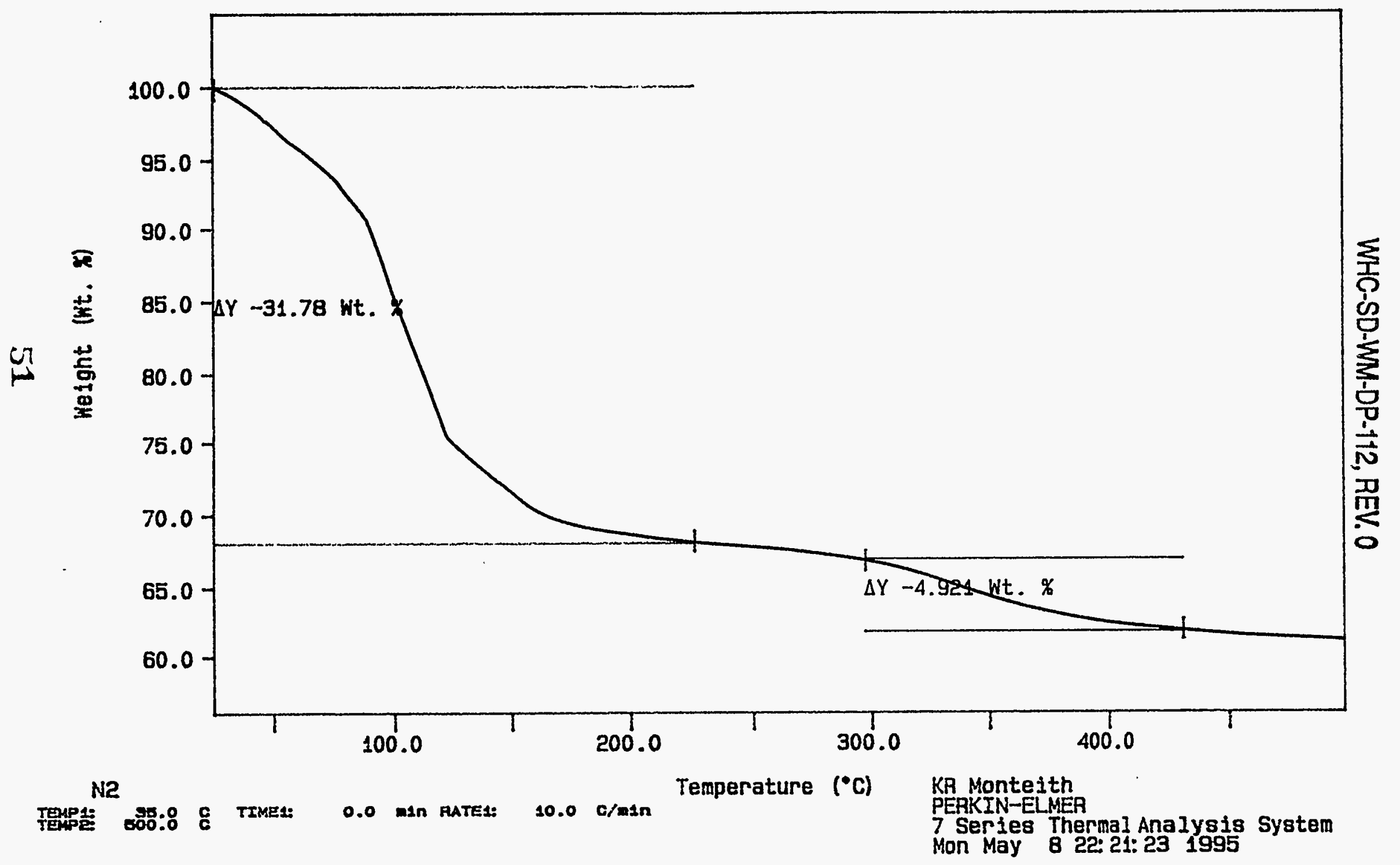


worklistrpt Version 2.0 02/21/95

05/03/95 11:33

WHC-SD-WM-DP-112, REV. 0

Page: 1

\section{LABCORE Data Entry Template for Worklist\# 1318}

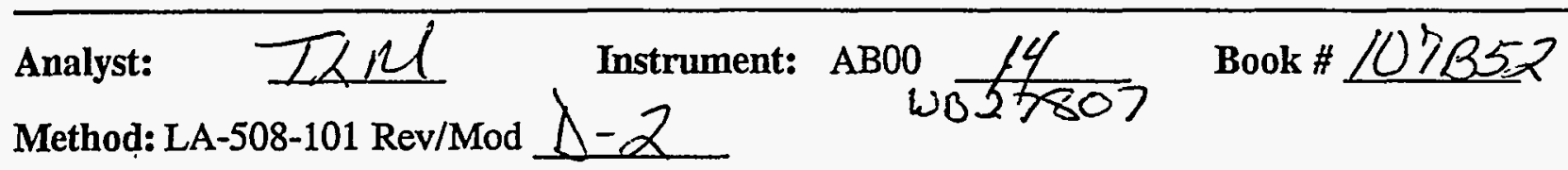

Worklist Comment: Run under RWP 273 if dose rate warrants. SLF

\begin{tabular}{|c|c|c|c|c|c|c|c|c|c|c|}
\hline \multirow[t]{6}{*}{ GROUP } & PROJECT & S TYPE & SAMPLE\# & RA & $\cdots$ & MATRIX & ACTUAL & FOUND & $D L$ & UNIT \\
\hline & & 1 STD & & & DALPHA01 ALPHA01 & SOLID & & & N/A & $u c i / g$ \\
\hline & & 1 sto & & & DALPHA0 1 ALPHA01E & SOLID & & & $N / A$ & \% Ct. Error \\
\hline & & 2 BLHK-PREP & & & QALPHA01 ALPHAOI & SOLID & & & $N / A$ & $\mathrm{uCi} / \mathrm{g}$ \\
\hline & & 2 BLNK-PREP & . & & DALPHA01 ALPHA01E & SOLID & & & N/A & \% Ct. Error \\
\hline & & 3 BLNK/BKG & .73 & & QALPHA01 ALPHA01 & SOLID & & & $N / A$ & $\mathrm{uCi} / \mathrm{g}$ \\
\hline 95000056 & $c-203$ & 4 SAMPLE & s95T000800 & $O F$ & QALPHA01 ALPHA01 & SOLID & N/A & & & $\mathrm{uCi} / \mathrm{g}$ \\
\hline 95000056 & $c-203$ & 4 SAMPLE & S95T000800 & $O F$ & DALPHA01 ALPHAO1E & SOLID & N/A & & & \% Ct. Error \\
\hline 95000056 & $c-203$ & 5 DUP & S95r000800 & OF & QALPHA01 ALPHA01 & SOLID & & & N/A & $u C i / g$ \\
\hline 95000056 & $C-203$ & 5 DUP. & s95T000800 & $O F$ & DALPHA01 ALPHA01E & SOLID & & & $N / A$ & \% ct. Error \\
\hline 95000056 & $c-203$ & 6 SPK & S95T000800 & $O F$ & QALPHA01 ALPHA01 & SOLID & & & $N / A$ & $\mathrm{uCi} / \mathrm{g}$ \\
\hline 95000056 & $C-203$ & 7 SAMPLE & $595 T 000804$ & $O F$ & DALPHA01 ALPHAO1 & SOLID & N/A & & & $u C i / g$ \\
\hline 95000056 & $c-203$ & 7 SAMPLE & $595 T 000804$ & $O F$ & DALPHA01 ALPHAOTE & SOLID & N/A & & 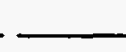 & \% ct. Error \\
\hline 95000056 & $c-203$ & 8 DUP & S95T000804 & $0 \mathrm{~F}$ & DALPHA01 ALPHA01 & SOLID & & & N/A & $u C i / g$ \\
\hline 95000056 & $c-203$ & 8 DUP & 5957000804 & $O F$ & DALPHA01 ALPHA01E & SOLIO & & & N/A & \% ct. Error \\
\hline
\end{tabular}

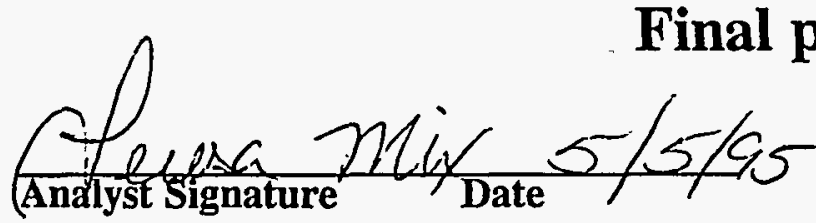

page for worklist \# 1318

$\frac{\text { Shan Lhalle } 5 \cdot P \cdot 95}{\text { Date }}$

Data Entry Comments: SPK recovery is out of lin its, Batch will Le rerun. ft zthel $5 / 9 / 95$

Units shown for $Q C$ (SPK \& STD) may not reflect the actual units. $D L=$ Detection Limit, $S=$ Worklist Slot Number,

$R=$ Replicate Number, $A=$ Aliquot Code. 


\begin{tabular}{|c|c|c|c|}
\hline \multicolumn{2}{|c|}{ AT : LA-508-101 (D-2) } & & \multirow[b]{2}{*}{ REPLICATE } \\
\hline \multicolumn{2}{|c|}{ LA-548-101 (A-3) } & STANDARD & \\
\hline Wo & DETECTOR NUMBER & $6.1 \%, 14$ & 3.114 \\
\hline STANDARD $\%$ & DISH SIZE & $W_{1}=.2$ & 2 \\
\hline 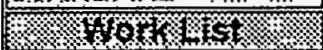 & TOTAL COUNTS & \%०\%4145 & ब. 4193 \\
\hline $6318 \%$ & COUNT TIME in MINUTES & $3 \%, 130$ & 30 \\
\hline \% $196 \% 18 \%$ & BACKGROUND in cpm & \%० \%००० & 0.5 \\
\hline AT $\quad \cdots \%$ & SAMPLE SIZE in $\mathrm{mL}$ & $2: \% 10,000$ & 10.000 \\
\hline 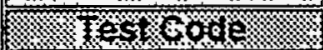 & DILUTION FACTOR & \%०. 1 & 1. \\
\hline @AkPKAOH. ? & DIGEST DILUTION FACTOR & ..8. 1 & 1 \\
\hline that+fix & EFFICIENCY FACTOR & 0.2274 & 0.2274 \\
\hline GQUD $: \%: \%$ & Le, Rmax, or Rs,(SAMPLE RATE) as APPROPRIATE & 137.667 & 139.267 \\
\hline $1>6 a m o l o l$ & Sample Concentration in $\mu \mathrm{Ci} / \mathrm{L}$ & 2.73E-02 & BOOK\# \\
\hline WRKLSTH T318\% & Replicate Concentration in $\mu \mathrm{Ci} / \mathrm{L}$ & $2.76 \mathrm{E}-02$ & 107852 \\
\hline 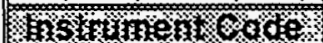 & & & \\
\hline WB27807 & Average Concentration in $\mu \mathrm{Ci} / \mathrm{L}$ & $2.7428 \mathrm{E}-02$ & \\
\hline 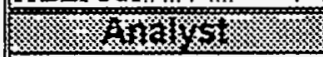 & & & \\
\hline TEM $:$ ४ & Rs (Sample Count Rate) $=(T C / C T)-B K G$ & & \\
\hline (2) & $=R s * 1000 \mathrm{~mL} \Omega * D F * D D F /(E F$ & 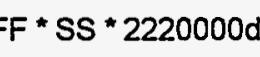 & i) \\
\hline $05105195,3,3$ & $=$ ALPHA TOTAL $\mu \mathrm{CI} / \mathrm{L} / 1000 \mathrm{~mL}$ & & \\
\hline (1) & $=[1](T h e$ Square Root of TC + BKG & I & CT)|] $]=1.96 * 100$ \\
\hline & & & \\
\hline
\end{tabular}

$\checkmark$ RESULTS $v$

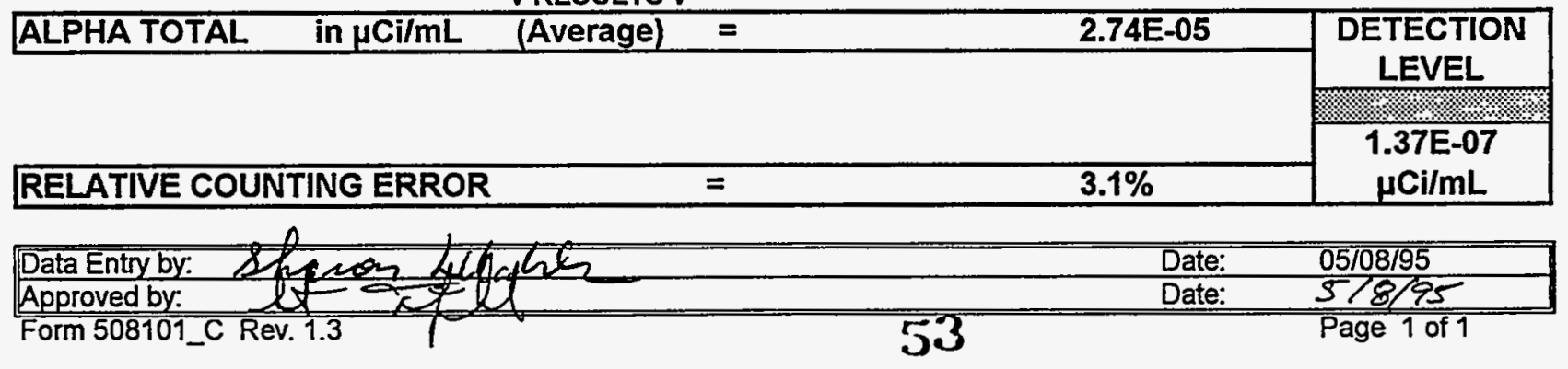




\begin{tabular}{|c|c|c|c|}
\hline \multicolumn{2}{|c|}{ AT: LA-508-101 (D-2) } & \multirow[b]{2}{*}{ BLANK } & \multirow[b]{2}{*}{ REPLICATE } \\
\hline \multicolumn{2}{|c|}{ LA-548-101 (A-3) } & & \\
\hline 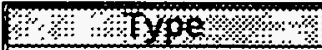 & DETECTOR NUMBER & $4 \% \cdots$ & .. $\quad 14$ \\
\hline BLANK & DISH SIZE 1,2, or 5 & 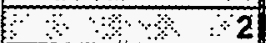 & 2 \\
\hline 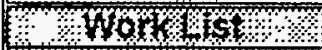 & TOTAL COUNTS & : 11 & $\because \therefore 11$ \\
\hline 1318 & COUNT TIME in MINUTES & 3.30 & 30 \\
\hline ATOH? & BACKGROUND in cpm & 0.0 .5 & 0.5 \\
\hline AT & SAMPLE SIZE in $\mathrm{mL}$ & \begin{tabular}{|l:l} 
& 0.100 \\
\end{tabular} & 0.100 \\
\hline Wescoode & DILUTION FACTOR & 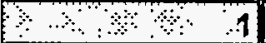 & 1 \\
\hline @ALPHAO1: & DIGEST GRAMS of SOLIDS/L & \%०े 1.986 & 1.986 \\
\hline \% & EFFICIENCY FACTOR & 0.2274 & 0.2274 \\
\hline SOLID & LC, Rmax, or Rs,(SAMPLE RATE) as APPROPRIATE & 0.301 & 0.301 \\
\hline WGamplo & Sample Concentration in $\mu \mathrm{Cilg}$ & $3.00 \mathrm{E}-03$ & BOOK\# \\
\hline S95T800 & Replicate Concentration in $\mu \mathrm{Ci} / \mathrm{g}$ & $3.00 \mathrm{E}-03$ & एँ \\
\hline Listruine & & & \\
\hline WB27807. & Maximum Concentration in $\mu \mathrm{Ci} / \mathrm{g}$ & $3.0047 E-03$ & \\
\hline \%कAlatyst & & & \\
\hline TLM & Rs (Sample Count Rate) $=(\mathrm{TC} / \mathrm{CT})-\mathrm{BKG}$ & & \\
\hline 0,0 Date & $=\mathrm{Rs} * 1000 \mathrm{~mL} / \cdot \mathrm{DF} /(\mathrm{EFF} \bullet \mathrm{SS}$ & $S \cdot D g / L \cdot 2220000$ & $/ / \mu \mathrm{Ci})$ \\
\hline 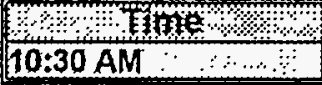 & $\begin{array}{l}\text { Relative Counting Error }=[\mid(T \text { The Square Root of TC }+ \text { BKG } \\
\text { Detection Levels and Less Than Values are determined from } P\end{array}$ & $\begin{array}{l}\left.{ }^{\circ} \mathrm{CT}\right) /(\mathrm{TC}-\mathrm{BK} \\
\text { rocedure LA-508- }\end{array}$ & (CT)I] $1.96 * 100$ \\
\hline
\end{tabular}

v RESULTS V

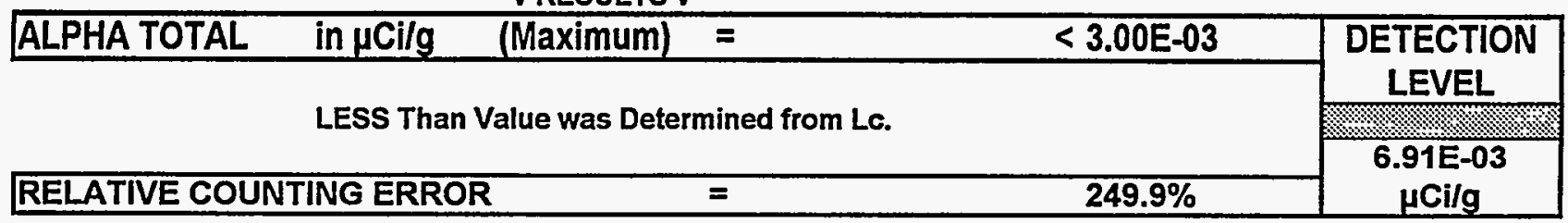

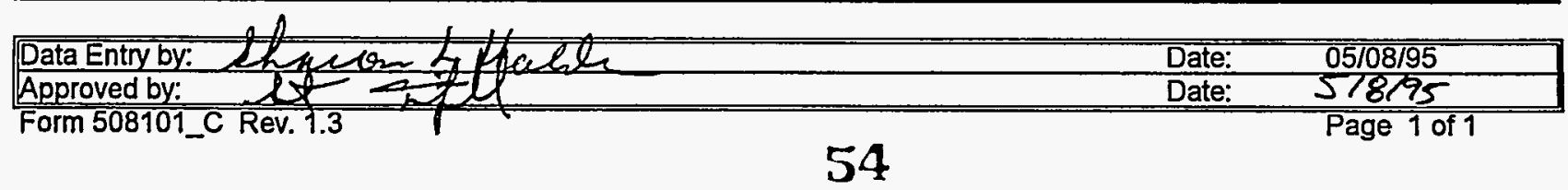


AT : LA-508-101 (D-2)

\section{LA-548-101 (A-3) SOLIDS}

\begin{tabular}{|c|c|c|c|}
\hline 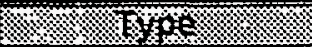 & DETECTOR NUMBER & $3 \times 14$ & का \\
\hline SAMPLE & DISH SIZE & - & 2 \\
\hline WOX & TOTAL COUNTS & $1 \% \%, 4406$ & 4582 \\
\hline $6318 \div \because \cdot 3$ & COUNT TIME in MINUTES & $\% \% 30$ & 30 \\
\hline $1 \times 000 \%$ & BACKGROUND in cpm & ४०: \% 0.5 & 0.5 \\
\hline AT $\leftrightarrow$ औी० & SAMPLE SIZE in $\mathrm{mL}$ & \% $\% 0,00$ & 0.100 \\
\hline $1 \times 0 s \mathrm{sog}$ & DILUTION FACTOR & $\% \%, \% 1$ & 1 \\
\hline @AEPHAO1 & DIGEST GRAMS of SOLIDS/L & $6, \%, 1986$ & 1.986 \\
\hline 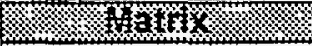 & EFFICIENCY FACTOR & 0.2274 & 0.2274 \\
\hline SOLLA: & Lc, Rmax, or Rs,(SAMPLE RATE) as APPROPRIATE & 136.367 & 152.233 \\
\hline Sacho & Sample Concentration in $\mu \mathrm{Ci} / \mathrm{g}$ & $1.36 \mathrm{E}+00$ & BOOK\# \\
\hline 5957800 & Replicate Concentration in $\mu \mathrm{Ci} / \mathrm{g}$ & $1.52 \mathrm{E}+00$ & $\because \because$ \\
\hline 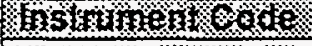 & & & \\
\hline WB27807, : & Average Concentration in $\mu \mathrm{Ci} / \mathrm{g}$ & $1.4393 E+00$ & \\
\hline Hatalds & \multirow{5}{*}{\multicolumn{3}{|c|}{ 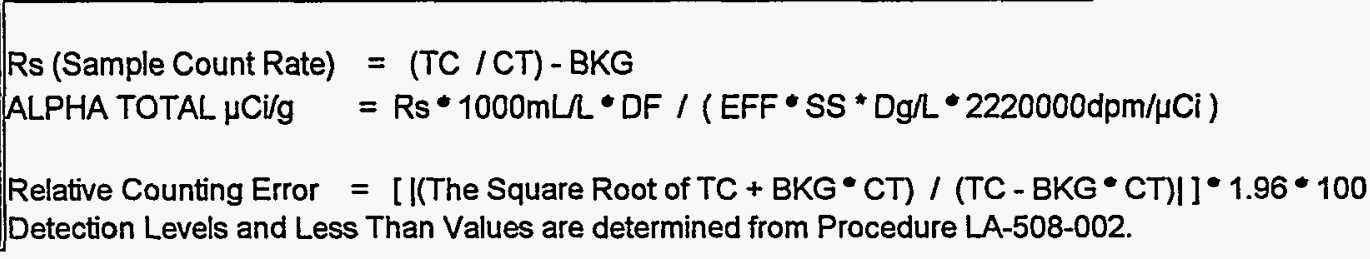 }} \\
\hline TEM & & & \\
\hline 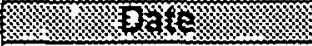 & & & \\
\hline 05105195,3 & & & \\
\hline H & & & \\
\hline
\end{tabular}

V RESULTS $v$

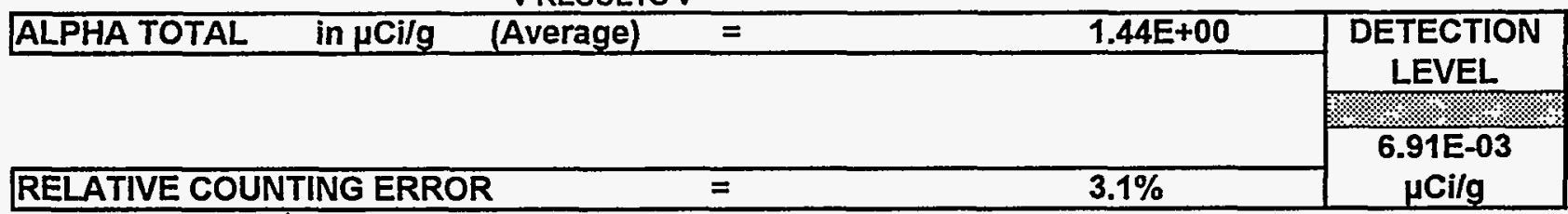

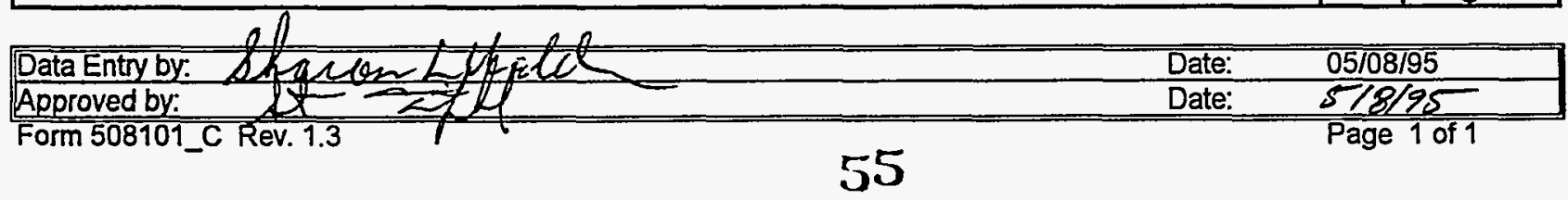




\begin{tabular}{|c|c|c|c|}
\hline \multicolumn{2}{|c|}{ AT : LA-508-101 (D-2) } & \multirow[b]{2}{*}{ SAMPLE } & \multirow[b]{2}{*}{ REPLICATE } \\
\hline LA-54 & $8-101(A-3)$ & & \\
\hline$\%$ WYpe & DETECTOR NUMBER & . 14 & $\therefore \therefore \therefore$ \\
\hline DUPLICATE: & DISH SIZE & $\begin{array}{r}4 \% \quad 2 \\
\end{array}$ & 2 \\
\hline Wom ms & TOTAL COUNTS & औे 4761 & 4494 \\
\hline 1318 & COUNT TIME in MINUTES & ४० 30 & 30 \\
\hline$\%$ ATor & BACKGROUND in cpm & औस & 0.5 \\
\hline AT. & SAMPLE SIZE in $\mathrm{mL}$ & 0.100 & 0.100 \\
\hline Tas & DILUTION FACTOR & 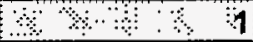 & 1 \\
\hline @ALPHAOI & DIGEST GRAMS of SOLIDS/L & $.2 \div 1.918$ & 1.918 \\
\hline Whatro & EFFICIENCY FACTOR & 0.2274 & 0.2274 \\
\hline SOLID: & LC, Rmax, or Rs, (SAMPLE RATE) as APPROPRIATE & 158.200 & 149.300 \\
\hline Sample & Sample Concentration in $\mu \mathrm{Ci} / \mathrm{g}$ & $1.63 E+00$ & BOOK\# \\
\hline S95T800: & Replicate Concentration in $\mu \mathrm{Cilg}$ & $1.54 \mathrm{E}+00$ & \% \\
\hline 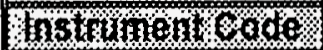 & & & \\
\hline WB27807\% & Average Concentration in $\mu \mathrm{Ci} / \mathrm{g}$ & $1.5879 E+00$ & \\
\hline Aftalys & & & \\
\hline TLM & Rs (Sample Count Rate) & & \\
\hline $\begin{array}{l}0 .: \text { Date } \\
0510.5195\end{array}$ & $s * 1000 \mathrm{~mL} / \cdot \mathrm{DF} /(\mathrm{EFF}$ & $S \cdot D g / L * 2220000$ & $\mathrm{~m} / \mu \mathrm{Ci})$ \\
\hline 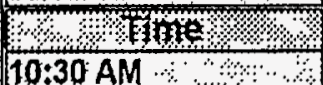 & $\begin{array}{l}\text { Relative Counting Error }=[\text { [ }(\text { The Square Root of } \mathrm{TC}+\mathrm{BK} \\
\text { Detection Levels and Less Than Values are determined from }\end{array}$ & $\begin{array}{l}\text { CT) / (TC - B } \\
\text { cedure LA-50 }\end{array}$ & CT) $\mid+1$ \\
\hline
\end{tabular}

VRESULTS V

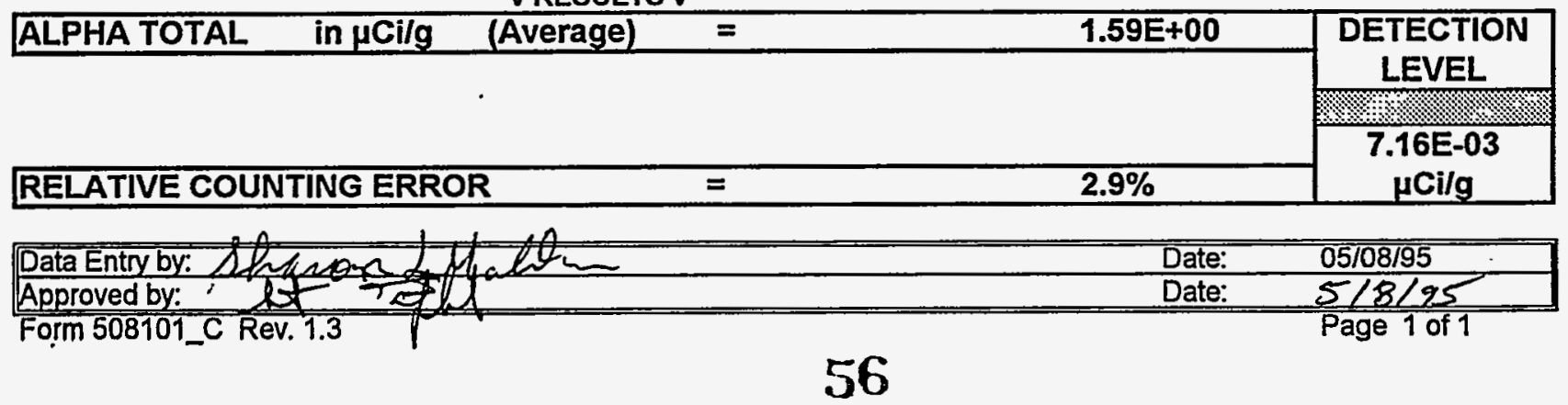


PLACE ANALYTICAL CARD IN BOX BELOW OR ATTACH TRAVELER

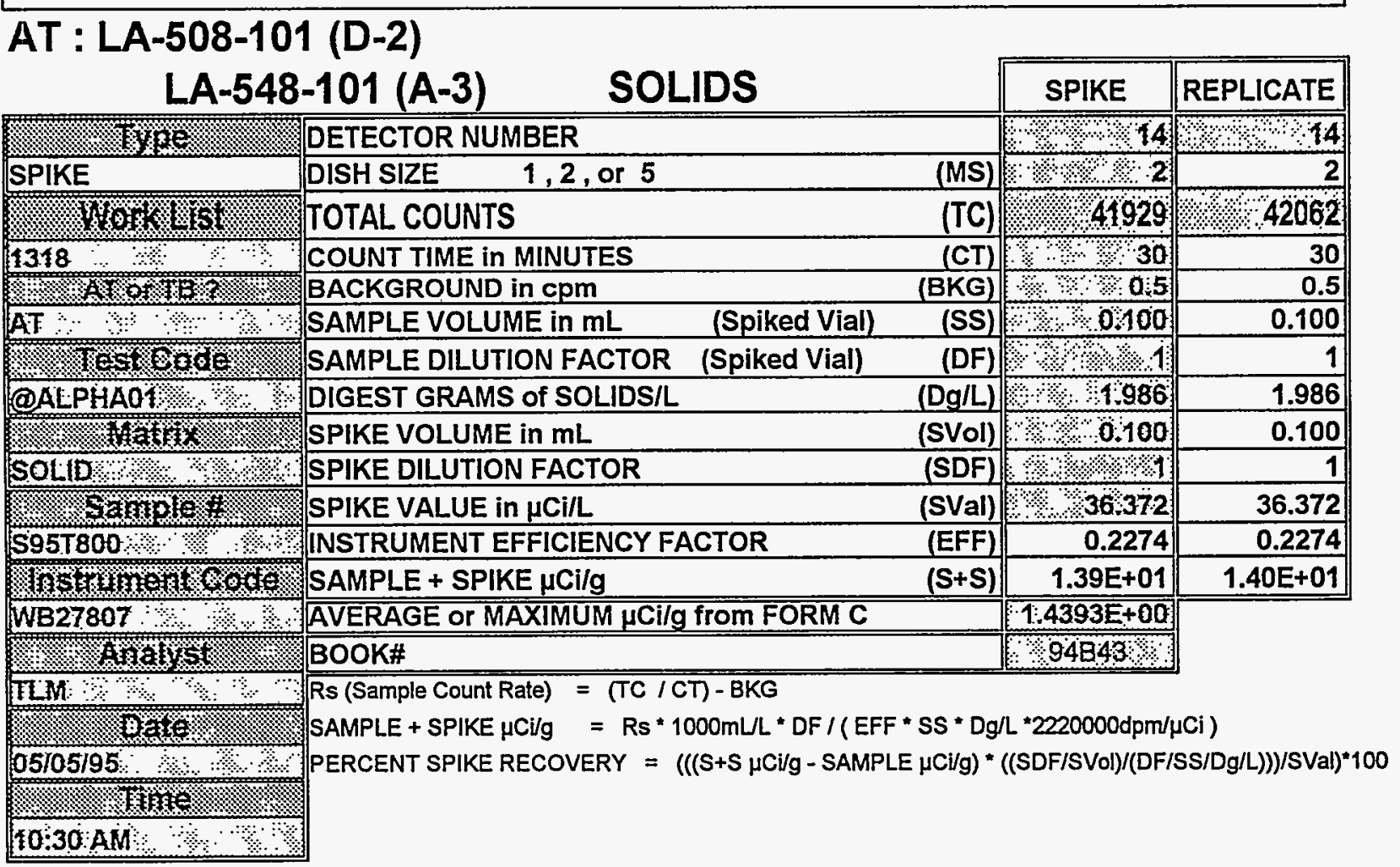

RESULT AVG. PERCENT SPIKE RECOVERY = $\quad 68.4 \%$

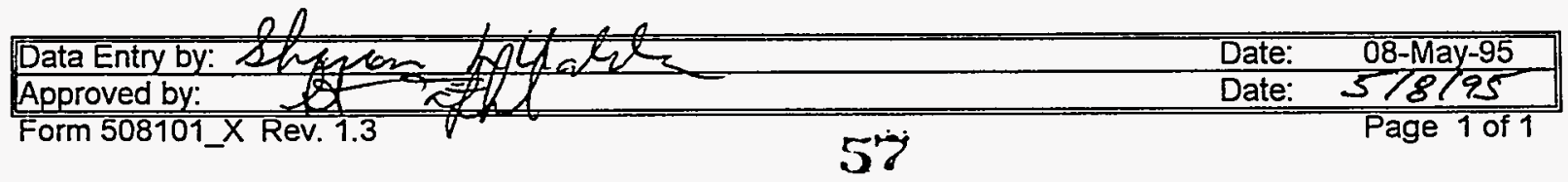




\section{AT : LA-508-101 (D-2)}

\section{LA-548-101 (A-3)}

SOLIDS

SAMPLE

18. TY98 7 DETECTOR NUMBER

SAMPLE: 1 DISH SIZE 1,2, or 5

W WORTCSY TOTAL COUNTS

4318

COUNT TIME in MINUTES

BFOTRE 2 BACKGROUND in cpm

AT $\cdots \ldots . .2$ SAMPLE SIZE in $\mathrm{mL}$

2. TESTCOOC DILUTION FACTOR

QALPHAOT

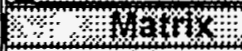

SOLID

DIGEST GRAMS Of SOLIDSIL

EFFICIENCY FACTOR

LC, Rmax, or Rs,(SAMPLE RATE) as APPROPRIATE

Famptar

Sample Concentration in $\mu \mathrm{Ci} / \mathrm{g}$

5957804

Replicate Concentration in $\mu \mathrm{Ci} / \mathrm{g}$

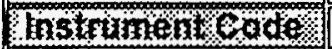

WB27807: .

Average Concentration in $\mu \mathrm{Ci} / \mathrm{g}$

\begin{tabular}{|c|c|}
\hline SAMPLE & REPLICATE \\
\hline $4: \because \quad: 14$ & 14 \\
\hline \%क: $\because 2$ & 2 \\
\hline 202200 & : $\quad 2089$ \\
\hline$\because \quad \fallingdotseq \mathbf{3 0}$ & 30 \\
\hline$\because 0.5$ & 0.5 \\
\hline $0_{0}, 0,100$ & 0.100 \\
\hline का & 1 \\
\hline $4,2.1224$ & 2.1224 \\
\hline 0.2274 & 0.2274 \\
\hline 72.833 & 69.133 \\
\hline $6.80 \mathrm{E}-01$ & BOOK\# \\
\hline 6.45E-01 & 3 \\
\hline
\end{tabular}

\% FAlaf

TLM $: \quad: \quad: 1$ (Sample Count Rate) = (TC/CT) - BKG

Date

$05 / 05 / 95$.

Relative Counting Error $=\left[\mid(T h e \text { Square Root of TC + BKG* CT) / (TC - BKG* CT)| }]^{\bullet} 1.96 * 100\right.$

10:30 AM

Detection Levels and Less Than Values are determined from Procedure LA-508-002.

v RESULTS $v$

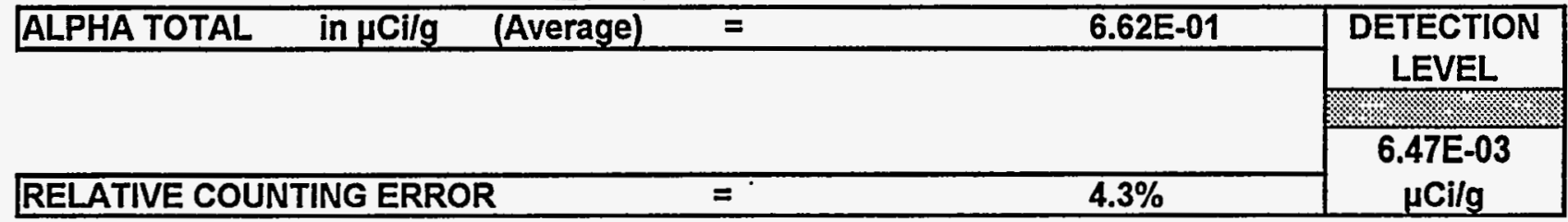

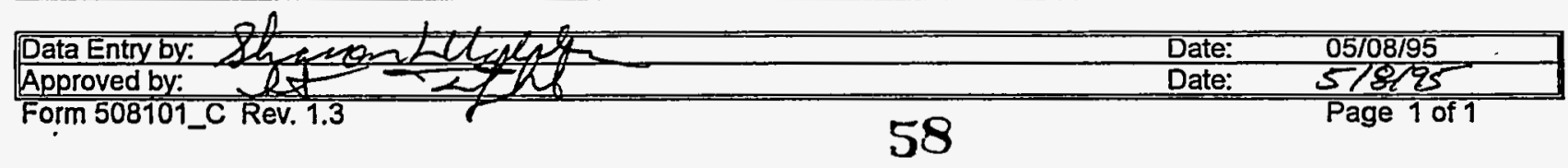


PLACE ANALYTICAL CARD IN BOX BELOW OR ATTACH TRAVELER WHC-SD-WM-DP-112, REV. 0

\begin{tabular}{|c|c|c|c|}
\hline \multicolumn{2}{|c|}{ AT : LA-508-101 (D-2) } & \multirow[b]{2}{*}{ SAMPLE } & \multirow[b]{2}{*}{ REPLICATE } \\
\hline LA-5 & 8-101(A-3) & & \\
\hline mese & DETECTOR NUMBER & \% & $1 \% \% \quad 14$ \\
\hline DUPLICATE & 1,2, or 5 & \%४७ 2 & 2 \\
\hline (6) & TOTAL COUNTS & $1.9+867$ & 1750 \\
\hline$\therefore \quad: \quad$ & COUNT TIME in MINUTES & 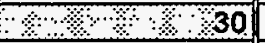 & 30 \\
\hline 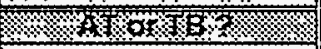 & BACKGROUND in cpm & $1 \%, 0.5$ & 0.5 \\
\hline AT $\%$ औे & SAMPLE SIZE in $\mathrm{mL}$ & $\% \% \% 0,100$ & 0.100 \\
\hline 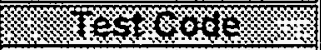 & DILUTION FACTOR & \%ै: 1 & 1 \\
\hline @AEPHAO1: & DIGEST GRAMS of SOLIDSIL & $8 \% .21548$ & 2.1548 \\
\hline 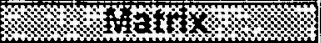 & EFFICIENCY FACTOR & 0.2274 & 0.2274 \\
\hline SOLID & Lc, Rmax, or Rs,(SAMPLE RATE) as APPROPRIATE & 61.733 & 57.833 \\
\hline (6) Samole & Sample Concentration in $\mu \mathrm{Ci} / \mathrm{g}$ & $5.68 \mathrm{E}-01$ & BOOK\# \\
\hline $595 T 804$ \% $\%$ \% & Replicate Concentration in $\mu \mathrm{Ci} / \mathrm{g}$ & 5.32E-01 & 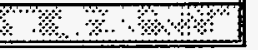 \\
\hline 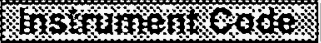 & & & \\
\hline WB27807 \% & Average Concentration in $\mu \mathrm{Ci} / \mathrm{g}$ & 5.4958E-01 & \\
\hline 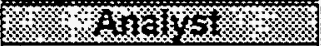 & & & \\
\hline TEM $, \%: \cdots$ & Rs (Sample Count Rate) $=(\mathrm{TC} / \mathrm{CT})-\mathrm{BKG}$ & & \\
\hline Mol & $=\mathrm{Rs} * 1000 \mathrm{mLL} \bullet \mathrm{DF} /(\mathrm{EFF}$ & $2220000 d$ & $\mathrm{pm} / \mu \mathrm{Ci})$ \\
\hline $05105195 \%$ & & & \\
\hline Fino & ounting Error $=[\mid($ The Sc & & CT) $] \cdot 1.96 \bullet 1$ \\
\hline 30 AM & e determined fro & & \\
\hline
\end{tabular}

V RESULTS V

\begin{tabular}{|lll|c|}
\hline ALPHA TOTAL in $\mu$ Ci/g (Average) $=$ & $5.50 E-01$ & $\begin{array}{c}\text { DETECTION } \\
\text { LEVEL }\end{array}$ \\
\hline RELATIVE COUNTING ERROR & $=$ & & $6.37 E-03$ \\
$\mu \mathrm{Ci} / \mathrm{g}$ \\
\hline
\end{tabular}

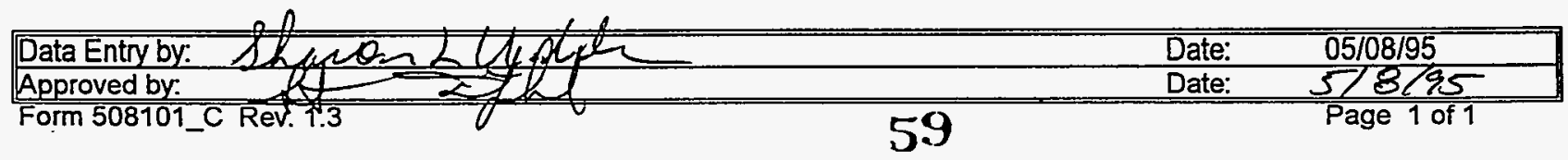




\section{LABCORE Data Entry Template for Worklist\# 1366}

Analyst: $\quad$ LMT $\quad$ Instrument: $A B 00 \frac{16}{\omega B 27806}$ Book 107 BS2

Method: LA-508-101 Rev/Mod $D-2$

Worklist Comment: Use a $.050 \mathrm{~mL}$ sample size. Rerun \#1. SLF

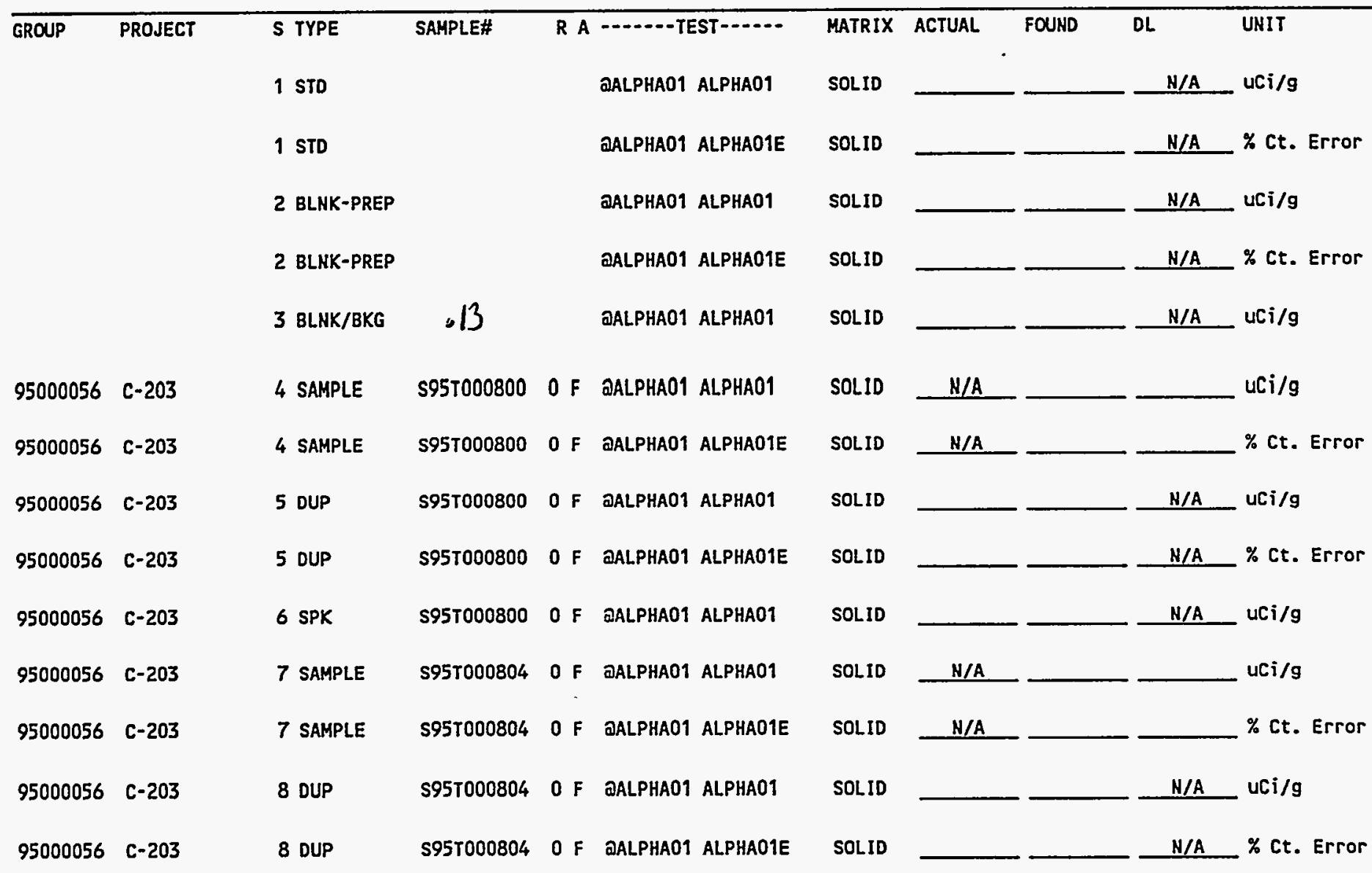

\section{Final page for worklist \# 1366}
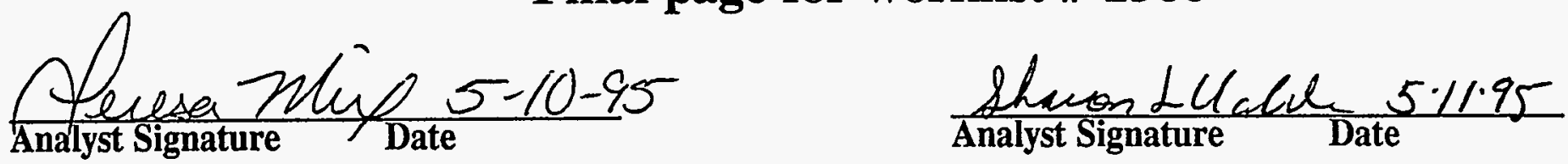

Data Entry Comments:

Data Eron ist run (worklist 1318) will be used ixstend of this Date, $14 / 15 / 95$

Units shown for $Q C$ (SPK \& STD) may not reflect the actual units. $D L=$ Detection Limit, $S=$ Worklist Slot Number,

$R=$ Replicate Number, $A=$ Aliquot Code. 


\begin{tabular}{|c|c|c|c|}
\hline \multicolumn{2}{|c|}{ AT : LA-508-101 (D-2) } & \multirow[b]{2}{*}{ STANDARD } & \multirow[b]{2}{*}{ REPLICATE } \\
\hline \multicolumn{2}{|c|}{ LA-548-101 (A-3) } & & \\
\hline M & DETECTOR NUMBER & $. \% \div 916$ & 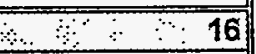 \\
\hline STANDARD & DISH SIZE $\quad 1,2$, or 5 & $3 \% 2$ & 2 \\
\hline maromstom & TOTAL COUNTS & 2.133931 & +6 3718 \\
\hline $1366 . \quad \therefore \quad \because$ & COUNT TIME in MINUTES & $0: 330$ & 30 \\
\hline 12forks & BACKGROUND in cpm & \%\% $\quad 0.4$ & 0.4 \\
\hline AT: & SAMPLE SIZE in $\mathrm{mL}$ & $3 \%, 10,000$ & 10.000 \\
\hline (1) 800 & DILUTION FACTOR & \%, $.1 \%$ & 1. \\
\hline @AEPHAO1 - & DIGEST DILUTION FACTOR & 6.1 .1 & 1 \\
\hline Wing & EFFICIENCY FACTOR & 0.2104 & 0.2104 \\
\hline LQUID & Lc, Rmax, or Rs, (SAMPLE RATE) as APPROPRIATE & 130.633 & 123.533 \\
\hline 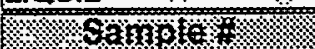 & Sample Concentration in $\mu \mathrm{Ci} / \mathrm{L}$ & $2.80 \mathrm{E}-02$ & BOOK\# \\
\hline WORKLIST\#1366\% & Replicate Concentration in $\mu \mathrm{Ci} / \mathrm{L}$ & 2.64E-02 & $107 B 52$ \\
\hline 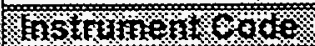 & & & \\
\hline WB27806 & Average Concentration in $\mu \mathrm{Cill}$ & $2.7208 \mathrm{E}-02$ & \\
\hline \%A A A & & & \\
\hline TLM : : $:-4$ & Rs (Sample Count Rate) & & \\
\hline 2010 & ALPHA TOTAL $\mu \mathrm{Ci} \Omega=\mathrm{Rs} * 1000 \mathrm{~mL} / \mathrm{DF} * \mathrm{DDF} /(\mathrm{E}$ & $\mathrm{FF}$ & \\
\hline $05110195, \cdots$ & $\begin{array}{l}\text { ALPHA TOTAL } \mu \mathrm{Ci} / \mathrm{mL}=\text { ALPHA TOTAL } \mu \mathrm{Cil} / 1000 \mathrm{~mL} \\
\text { Relative Counting Error }=[\mid(T \text { The Square Root of TC }+ \text { BKC }\end{array}$ & & \\
\hline $0: 15 \mathrm{AM}$ & & & \\
\hline
\end{tabular}

v RESULTS $v$

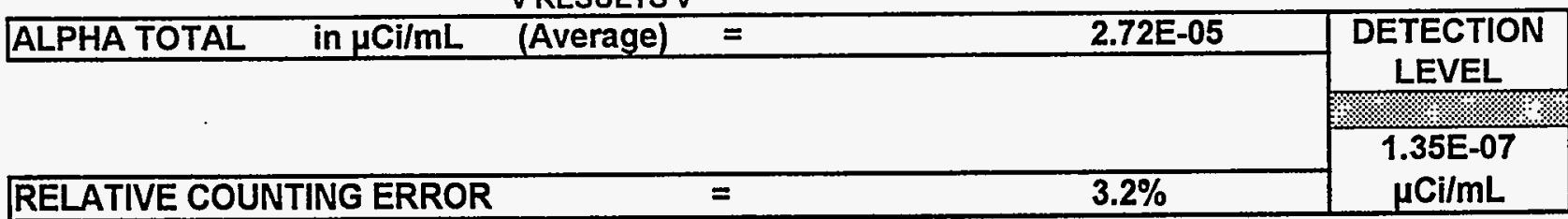

\begin{tabular}{|lll|}
\hline Data Entry by: Shate: & Date: & $05 / 11 / 95$ \\
\hline Approved by: & Date: & $5 / 11 / 95$ \\
\hline Form 508101_C Rev. 1.3 & Page 1 of 1
\end{tabular}

61 


\begin{tabular}{|c|c|c|c|}
\hline \multicolumn{4}{|c|}{ AT : LA-508-101 (D-2) } \\
\hline & DETECTOR NUMBER & $2 \% 16$ & 16 \\
\hline BEANK. & 1,2, or 5 & $\therefore \cdot 1.2$ & 2 \\
\hline 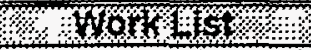 & TOTAL COUNTS & 32,1 & : \\
\hline m & COUNT TIME in MINUTES & 3.30 & 30 \\
\hline \% $1001 B \%$ & BACKGROUND in cpm & 0.40 .4 & 0.4 \\
\hline \% & SAMPLE SIZE in $\mathrm{mL}$ & 0.050 & 0.050 \\
\hline TESAOOCR & DILUTION FACTOR & $\% \quad 1$ & 1 \\
\hline @ALPHAOT & DIGEST GRAMS of SOLIDSIL & $\% 1.986$ & 1.986 \\
\hline 6 maffx: & EFFICIENCY FACTOR & 0.2104 & 0.2104 \\
\hline SOLID : & Lc, Rmax, or Rs,(SAMPLE RATE) as APPROPRIATE & 0.269 & 0.269 \\
\hline Samplë: & Sample Concentration in $\mu \mathrm{Ci} / \mathrm{g}$ & 5.81E-03 & BOOK\# \\
\hline \$95T800 & Replicate Concentration in $\mu \mathrm{Cilg}$ & $5.81 \mathrm{E}-03$ & $: \cdots$ \\
\hline Hotrumentodo & & & \\
\hline MB27806 & Maximum Concentration in $\mu \mathrm{Ci} / \mathrm{g}$ & 5.8093E-03 & \\
\hline \%Analysk & & & \\
\hline TLM & Rs (Sample Count Rate) $=(\mathrm{TC} / \mathrm{CT})-\mathrm{BKG}$ & & \\
\hline H Oate \% & $=R s^{*} 1000 \mathrm{mLL} * \mathrm{DF} /\left(E F F{ }^{*} \mathrm{~s}\right.$ & $S \cdot D g h$ & $\mathrm{om} / \mu \mathrm{Ci})$ \\
\hline $05 / 10 / 95$ & & & \\
\hline 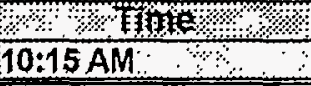 & $\begin{array}{l}\text { Relative Counting Error }=[\text { I }(T \text { The Square Root of } T C+B K G \\
\text { Detection Levels and Less Than Values are determined from } \mathrm{P}\end{array}$ & $\left.{ }^{\circ} \mathrm{CT}\right) /(\mathrm{T}$ & (CT) \\
\hline
\end{tabular}

V RESULTS V

ALPHA TOTAL (Maximum)

$=$

$<5.81 E-03$

LESS Than Value was Determined from Lc. 


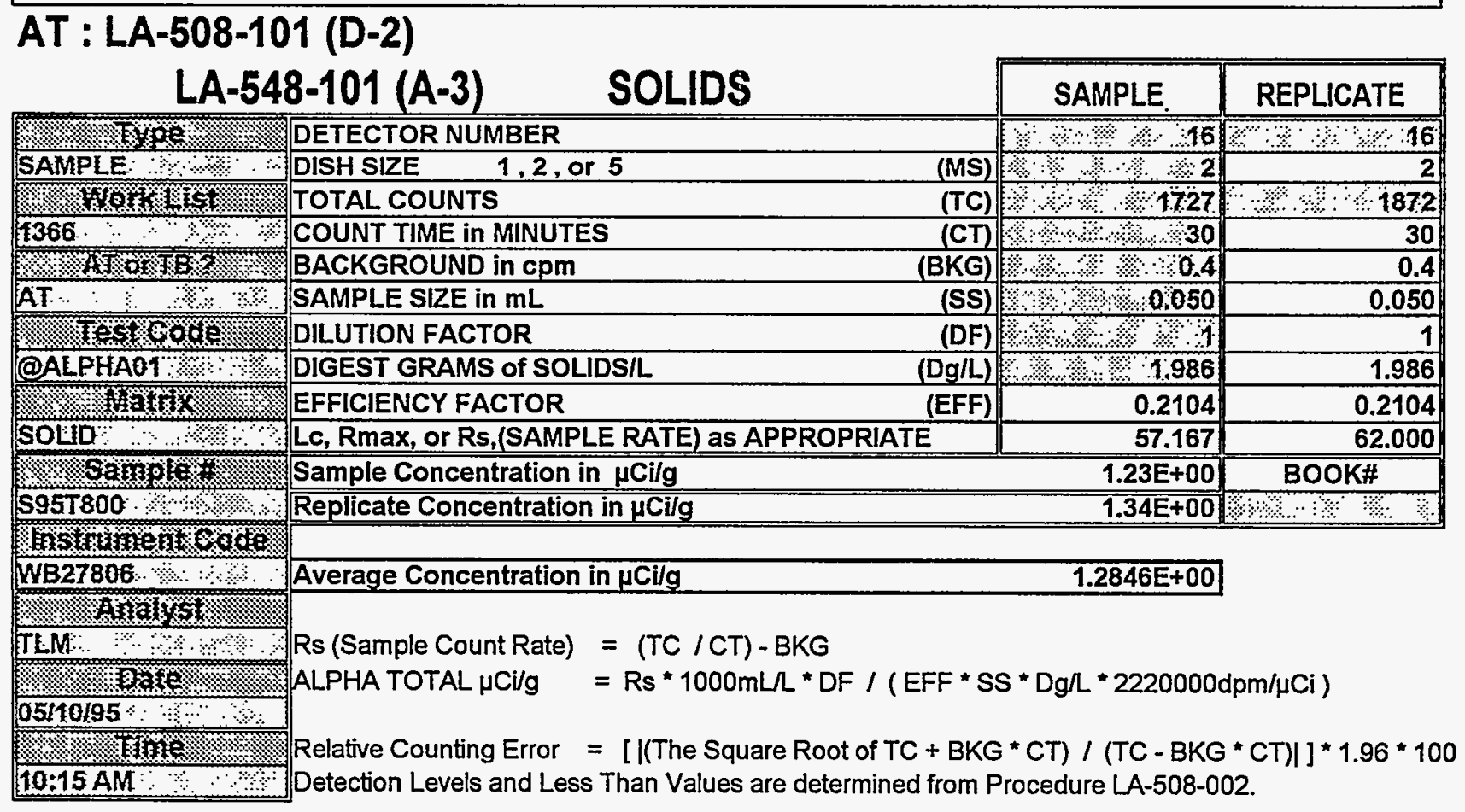

v RESULTS v

\begin{tabular}{|llc|c|}
\hline ALPHA TOTAL in $\mu \mathrm{Ci} / \mathrm{g}$ (Average) $=$ & $1.28 \mathrm{E}+00$ & $\begin{array}{c}\text { DETECTION } \\
\text { LEVEL }\end{array}$ \\
\hline RELATIVE COUNTING ERROR & $=$ & $4.8 \%$ & $1.36 \mathrm{E}-02$ \\
$\mu \mathrm{Ci} / \mathrm{g}$ \\
\hline
\end{tabular}

\begin{tabular}{|lll|}
\hline Data Entry by: & Date: & $05 / 11 / 95$ \\
Approved by: & Date: & $5 / 1 / 25$ \\
\hline Forim 508101_C Rev. 1.3 & 63 & Page 1 of 1
\end{tabular}


PLACE ANALYTICAL CARD IN BOX BELOW OR ATTACH TRAVELER . WHC-SD-WM-DP-112, REV. 0

\begin{tabular}{|c|c|c|c|}
\hline \multicolumn{2}{|c|}{ AT : LA-508-101 (D-2) } & \multirow[b]{2}{*}{ SAMPLE } & \multirow[b]{2}{*}{ REPLICATE } \\
\hline \multicolumn{2}{|c|}{ LA-548-101 (A-3) } & & \\
\hline \% $\%$ ye\% & DETECTOR NUMBER & 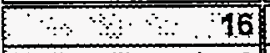 & 16 \\
\hline DUPLICATE: & DISH SIZE $\quad 1,2$, or 5 & : $: 2$ & 2 \\
\hline WOKKS & TOTAL COUNTS & $\because 3,1944$ & 2309 \\
\hline 1366 & COUNT TIME in MINUTES & \begin{tabular}{|l|}
3 \\
\end{tabular} & 30 \\
\hline 20T\% $1 \mathrm{~TB}$ & BACKGROUND in cPm & की $: 0.4$ & 0.4 \\
\hline AT: & SAMPLE SIZE in $\mathrm{mL}$ & $1 \% 0,050$ & 0.050 \\
\hline$\% 16500$ & DILUTION FACTOR & ४ै। & 1 \\
\hline QALPHAO 1 : & DIGEST GRAMS of SOLIDS/L & 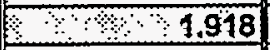 & 1.918 \\
\hline Hexmatrx & EFFICIENCY FACTOR & 0.2104 & 0.2104 \\
\hline SOLID & LC, Rmax, or Rs,(SAMPLE RATE) as APPF & 64.400 & 76.567 \\
\hline Sample $4:$ & Sample Concentration in $\mu \mathrm{Ci} / \mathrm{g}$ & 1.44E+00 & BOOK\# \\
\hline S95T800 & Replicate Concentration in $\mu \mathrm{Ci} / \mathrm{g}$ & $1.71 \mathrm{E}+00$ & 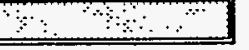 \\
\hline 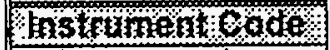 & & & \\
\hline WB27806 & Average Concentration in $\mu \mathrm{Ci} / \mathrm{g}$ & $1.5735 E+00$ & \\
\hline \&Analy & & & \\
\hline TLM,$\cdots$, & Rs (Sample Count Rate) $=(T C / C T)-B K G$ & & \\
\hline \%०० & $=R s * 1000 \mathrm{mLL} * \mathrm{DF} /(\mathrm{EFF} * \mathrm{~S}$ & $S^{*} \mathrm{Dg} / \mathrm{L} \cdot 2220000 \mathrm{C}$ & $\mu \mathrm{Ci})$ \\
\hline $05 / 10 / 95$ & & & \\
\hline$\frac{10: 15 \mathrm{AM}}{10}$ & ss 7 & & - \\
\hline
\end{tabular}

V RESULTS $v$

\begin{tabular}{|llll|c|}
\hline ALPHA TOTAL in $\mu$ Ci/g & (Average) & $=$ & $1.57 E+00$ & $\begin{array}{c}\text { DETECTION } \\
\text { LEVEL }\end{array}$ \\
& & & & \\
\hline RELATIVE COUNTING ERROR & $=$ & $4.5 \%$ & $1.41 \mathrm{E}-02$ \\
$\mu \mathrm{Ci} / \mathrm{g}$ \\
\hline
\end{tabular}

\begin{tabular}{|lll|}
\hline Data Entry by: Affecon & & \\
\hline Approved by: & Date: & $05 / 11 / 95$ \\
\hline Form 508101_C Rev. 1.3 & Date: & $5 / 11 / 95$ \\
\hline
\end{tabular}




\section{AT : LA-508-101 (D-2)}

\begin{tabular}{|c|c|c|c|}
\hline \multirow{2}{*}{\multicolumn{2}{|c|}{ LA-548-101 (A-3) }} & \multirow{3}{*}{ SPIKE } & \multirow{3}{*}{\begin{tabular}{|l|} 
REPLICATE \\
16 \\
\end{tabular}} \\
\hline & & & \\
\hline 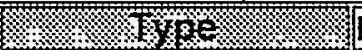 & DETECTOR NUMBER & & \\
\hline SPIKE & DISH SIZE & ० 2 & 2 \\
\hline WON & TOTAL COUNTS & 27059 & 332081 \\
\hline $1366 \quad \% \quad \%$ & COUNT TIME in MINUTES & $\% \longdiv { 3 0 }$ & 30 \\
\hline 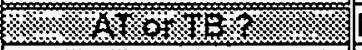 & BACKGROUND in cpm & 0,4 & 0.4 \\
\hline AT $: \cdots$ & SAMPLE VOLUME in $\mathrm{mL}$ & $2 \% 00050$ & 0.050 \\
\hline 150syogor & SAMPLE DILUTION FACTOR (Spiked Vial) & \% $: 1$ & 1 \\
\hline QALPHAOT: : & DIGEST GRAMS of SOLIDS/L & $8 \longdiv { 1 9 8 6 }$ & 1.986 \\
\hline 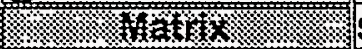 & SPIKE VOLUME in $\mathrm{mL}$ & $\% \% 0.100$ & 0.100 \\
\hline SOLAD $\% 1 \%$ & SPIKE DILUTION FACTOR & \%,\%\%, & 1 \\
\hline 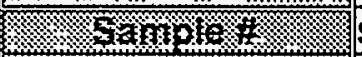 & SPIKE VALUE in $\mu \mathrm{Ci} / \mathrm{L}$ & $.2 .36: 372$ & 36.372 \\
\hline $5957800 \%$ & INSTRUMENT EFFICIENCY FACTOR & 0.2104 & 0.2104 \\
\hline 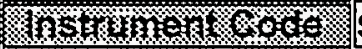 & SAMPLE + SPIKE $\mu \mathrm{Ci} / \mathrm{g}$ & $1.94 E+01$ & $2.30 \mathrm{E}+01$ \\
\hline WB27806 & AVERAGE or MAXIMUM $\mu \mathrm{Ci} / \mathrm{g}$ from FORM C & $1: 2846 \mathrm{E}+00$ & \\
\hline (1) & BOOK\# & $994 \mathrm{~B} 43$ & \\
\hline \multicolumn{4}{|c|}{ Rs (Sample Count Rate) } \\
\hline 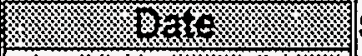 & \multirow{3}{*}{\multicolumn{3}{|c|}{ 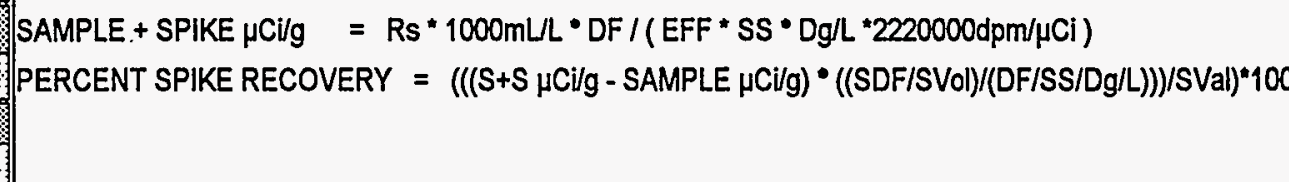 }} \\
\hline 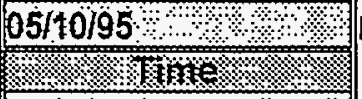 & & & \\
\hline 10:15 AM: & & & \\
\hline
\end{tabular}

\section{RESULT AVG. PERCENT SPIKE RECOVERY = $=54.5 \%$}

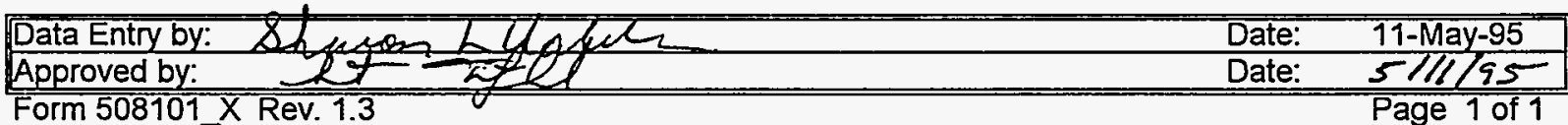




\begin{tabular}{|c|c|c|c|}
\hline \multicolumn{2}{|c|}{ AT : LA-508-101 (D-2) } & \multirow[b]{2}{*}{ SAMPLE } & \multirow[b]{2}{*}{ REPLICATE } \\
\hline LA-5 & $8-101(A-3)$ & & \\
\hline W Y Y & DETECTOR NUMBER & $\because \cdots 16$ & 16 \\
\hline SAMPLE & DISH SIZE & $1 \%$ & 2 \\
\hline Worktst & TOTAL COUNTS & 1041 & $\therefore 1093$ \\
\hline $4366 \quad-1$ & COUNT TIME in MINUTES & $\because \quad 230$ & 30 \\
\hline HTOX IBK & BACKGROUND in cpm & $\because \quad-0.4$ & 0.4 \\
\hline$A T \quad \therefore \quad \therefore \quad$ & SAMPLE SIZE in $\mathrm{mL}$ & $\therefore 0.050$ & 0.050 \\
\hline Testoode & DILUTION FACTOR & अ 1 & 1 \\
\hline @ALPHA01: & DIGEST GRAMS of SOLIDS/L & 21224 & 2.1224 \\
\hline matax & EFFICIENCY FACTOR & 0.2104 & 0.2104 \\
\hline SOLID & LC, Rmax, or Rs,(SAMPLE RATE) as APPROPRIATE & 34.300 & 36.033 \\
\hline Samplex & Sample Concentration in $\mu \mathrm{Cilg}$ & 6.92E-01 & BOOK\# \\
\hline $595 T 804$ & Replicate Concentration in $\mu \mathrm{Ci} / \mathrm{g}$ & 7.27E-01 & 8 \\
\hline 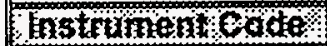 & & & \\
\hline WB27806: & Average Concentration in $\mu \mathrm{Ci} / \mathrm{g}$ & 7.0947E-01 & \\
\hline :Amaty & & & \\
\hline & Rs (Sample Count Rate) $=(T C / C T)-B K G$ & & \\
\hline की & $=R s * 1000 \mathrm{mLL} * \mathrm{DF} /(\mathrm{EFF} * \mathrm{~s}$ & * Dg/ * 2220000d & $\mathrm{m} / \mu \mathrm{Ci})$ \\
\hline $05 / 10 / 95$ & The Square Root of TC+ & & \\
\hline M & & $a 1$ & \\
\hline
\end{tabular}

v RESULTS V

\begin{tabular}{|llc|c|}
\hline ALPHA TOTAL in $\mu$ Ci/g (Average) $=$ & $7.09 E-01$ & $\begin{array}{c}\text { DETECTION } \\
\text { LEVEL }\end{array}$ \\
\hline RELATIVE COUNTING ERROR & $=$ & & $1.27 E-02$ \\
& & $6.2 \%$ & $\mu \mathrm{Ci} / \mathrm{g}$ \\
\hline
\end{tabular}

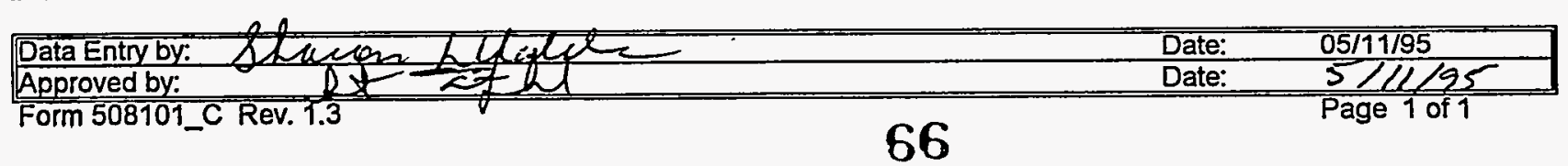


PLACE ANALYTICAL CARD IN BOX BELOW OR ATTACH TRAVELER WHC-SD-WM-DP-112, REV. 0

\begin{tabular}{|c|c|c|c|}
\hline \multicolumn{2}{|c|}{ AT : LA-508-101 (D-2) } & \multirow[b]{2}{*}{ SAMPLE } & \multirow[b]{2}{*}{ REPLICATE } \\
\hline \multicolumn{2}{|c|}{ LA-548-101 (A-3) } & & \\
\hline He & DETECTOR NUMBER & \%०\%16 & ४७ै\% \\
\hline DUPLICATE & 1,2, or 5 & ..\%., 2 & 2 \\
\hline 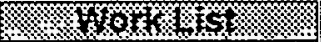 & TOTAL COUNTS &.$\% 11009$ & 957. \\
\hline $1366 . \%$ & COUNT TIME in MINUTES & ०४४\%:30 & 30 \\
\hline $1.67 \% 918 \%$ & BACKGROUND in cpm & $\% \% \%, 0.4$ & 0.4 \\
\hline AT $\because \because \% \%$ & SAMPLE SIZE in $\mathrm{mL}$ & $1, \%: 0,050$ & 0.050 \\
\hline 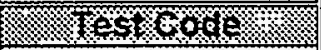 & DILUTION FACTOR & \%०.: : 1 & 1 \\
\hline QAEPHAOI 14 & DIGEST GRAMS of SOLIDSIL & \%.,.2.7548 & 2.1548 \\
\hline 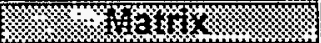 & EFFICIENCY FACTOR & 0.2104 & 0.2104 \\
\hline SOLFE: & LC, Rmax, or Rs,(SAMPLE RATE) as APPROPRIATE & 33.233 & 31.500 \\
\hline Sabuidol & Sample Concentration in $\mu \mathrm{Cilg}$ & 6.60E-01 & BOOK\# \\
\hline $595 T 804 \%$ & Replicate Concentration in $\mu \mathrm{Ci} / \mathrm{g}$ & 6.26E-01 & ४०० \\
\hline 符 & & & \\
\hline WB27806: : $: \%$ & Average Concentration in $\mu \mathrm{Ci} / \mathrm{g}$ & 6.4316E-01 & \\
\hline A & & & \\
\hline TEM $: \because: \because$ & Rs (Sample Count Rate) $=(\mathrm{TC} / \mathrm{CT})-\mathrm{BKG}$ & & \\
\hline (1) 120 & $=\mathrm{Rs}^{\bullet} 1000 \mathrm{~mL} / \mathrm{L}^{\bullet} \mathrm{DF} /(\mathrm{EFF}$ & $S \cdot D g / L \bullet 222000$ & $\mathrm{~m} / \mu \mathrm{Ci})$ \\
\hline $05110195: \cdots \cdots$ & & & \\
\hline 10:15:AM: & Relative Counting Error = & 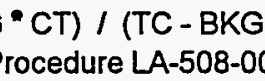 & שט. \\
\hline
\end{tabular}

V RESULTS V

\begin{tabular}{|lll|c|}
\hline ALPHA TOTAL in $\mu \mathrm{Ci} / \mathrm{g}$ (Average) $=$ & $6.43 \mathrm{E}-01$ & $\begin{array}{c}\text { DETECTION } \\
\text { LEVEL }\end{array}$ \\
\hline RELATIVE COUNTING ERROR & $=$ & & \\
\hline
\end{tabular}

\begin{tabular}{|c|c|c|}
\hline Data Entry by: & Date: & $05 / 11 / 95$ \\
\hline Approved by: & Date: & $5 / 11 / 85$ \\
\hline Form 508101_C Rev. 1.3 & & Page 1 of 1 \\
\hline
\end{tabular}




\section{LABCORE Data Entry Template for Worklist\# 1367}

Page: 1

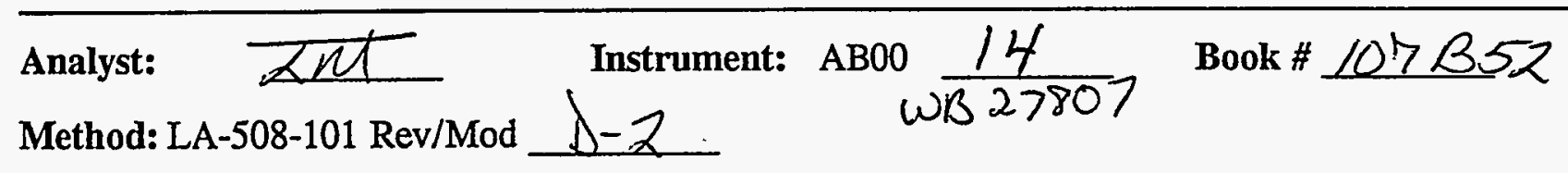

Worklist Comment: Use a $.050 \mathrm{~mL}$ sample size. Rerun \#1. SLF

\begin{tabular}{|c|c|c|c|c|c|c|c|c|c|c|}
\hline \multirow[t]{6}{*}{ GROUP } & PROJECT & S TYPE & SAMPLE\# & RA & - $-\cdots$ - TEST $-\cdots .$. & MATRIX & ACTUAL & FOUND & $\overline{D L}$ & UNIT \\
\hline & & 1 STD & & & DALPHA01 ALPHA01 & SOLID & & & N/A & $u C i / g$ \\
\hline & & 1 STD & & & QALPHA01 ALPHA01E & SOLID & & & $\mathrm{N} / \mathrm{A}$ & \% Ct. Error \\
\hline & & 2 BLLK-PREP & & & DALPHA0I ALPHAO1 & SOLID & & & $\mathrm{N} / \mathrm{A}$ & $\mathrm{uCi} / \mathrm{g}$ \\
\hline & & 2 BLNK-PREP & & & DALPHA01 ALPHA01E & SOLID & & & N/A & \% ct. Error \\
\hline & & 3 BLNK/BKG & & & DALPHA01 ALPHA01 & SOLIO & & & $N / A$ & $-u C i / g$ \\
\hline 95000056 & $c-203$ & 4 SAMPLE & s95T000808 & O F & DALPHA01 ALPHA01 & SOLID & N/A & & & uCi/g \\
\hline 95000056 & $c-203$ & 4 SAMPLE & S95T000808 & O F & DALPHA01 ALPHA01E & SOLID & $\mathrm{N} / \mathrm{A}$ & & & \% ct. Error \\
\hline 95000056 & $C-203$ & 5 DUP & $\$ 95 T 000808$ & O F & DALPHA01 ALPHA01 & SOLID & & & N/A & $u C i / g$ \\
\hline 95000056 & $C-203$ & 5 DUP & $595 T 000808$ & $O F$ & DALPHA01 ALPHA01E & SOLID & & & N/A & \% ct. Error \\
\hline 95000056 & $C-203$ & 6 SAMPLE & S95T000812 & OF & DALPHA01 ALPHA01 & SOLID & $N / A$ & & & $u C i / g$ \\
\hline 95000056 & $C-203$ & 6 SAMPLE & s95T000812 & $O F$ & DALPHA01 ALPHA01E & SOLID & $H / A$ & & & \% ct. Error \\
\hline 95000056 & $c-203$ & 7 DUP & s95T000812 & $O F$ & DALPHA01 ALPHA01 & SOLID & - & & N/A & $\mathrm{uCi} / \mathrm{g}$ \\
\hline 95000056 & $c-203$ & 7 DUP & s95T000812 & OF & DALPHA01 ALPHA01E & SOLID & & & N/A & $\%$ Ct. Erro \\
\hline
\end{tabular}

\section{Final page for worklist \# 1367}
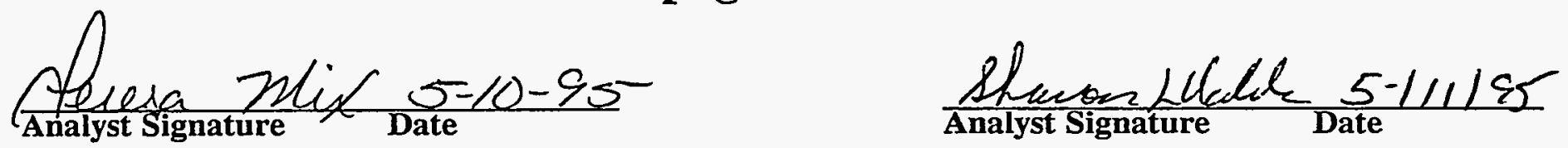

Data Entry Comments:

High RPD values are consistet with original run. Indicates a proble w with seple prep (possibly that the euplicates were interehoged). ft wh/ $5 / 15 / 55$

Units shown for $Q C$ (SPK \& STD) may not reflect the actual units. $D L=$ Detection Limit, $S=$ Worklist Slot Number, $R=$ Replicate Number, $A=$ Aliquot Code. 
PLACE ANALYTICAL CARD IN BOX BELOW OR ATTACH TRAVELER WHC-SD-WM-DP-112, REV. 0

\begin{tabular}{|c|c|c|c|}
\hline \multicolumn{4}{|c|}{ AT : LA-508-101 (D-2) } \\
\hline \multicolumn{2}{|c|}{ LA-548-101 (A-3) } & STANDARD & REPLICATE \\
\hline (1) & DETECTOR NUMBER & \%.\%. 14 & : $: 14$ \\
\hline STANDARD: & DISH SIZE & ৮৮৮ \% & $\overline{2}$ \\
\hline 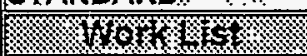 & TOTAL COUNTS & \%.\%. 4212 & \% 4042 \\
\hline $1367 \cdot \cdots \times \cdot 4$ & COUNT TIME in MINUTES & $1.6 \% 30$ & 30 \\
\hline . & BACKGROUND in cpm & $0 \% \odot \% 03$ & 0.3 \\
\hline$\therefore$. & SAMPLE SIZE in $\mathrm{mL}$ & $\because \%, 10 ; 000$ & 10.000 \\
\hline 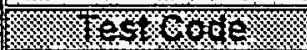 & DILUTION FACTOR & \%० ४४ 1 & 1 \\
\hline @AEPHAO 1: : & DIGEST DILUTION FACTOR & B. $: 3.1$ & 1 \\
\hline 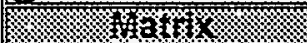 & EFFICIENCY FACTOR & 0.2274 & 0.2274 \\
\hline LQUID $\because$. & Lc, Rmax, or Rs,(SAMPLE RATE) as APPROPRIATE & 140.100 & 134.433 \\
\hline Sariole & Sample Concentration in $\mu \mathrm{Ci} / \mathrm{L}$ & $2.78 \mathrm{E}-02$ & BOOK\# \\
\hline WORKLIST\#1367 & Replicate Concentration in $\mu \mathrm{Ci}$ & 2.66E-02 & $107 \mathrm{~B} 52$ \\
\hline
\end{tabular}

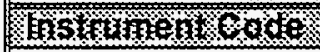

YB27.807 $\%$ Average Concentration in $\mu \mathrm{Ci} / \mathrm{L}$

2.7191E-02

I ATH

TLM

mas

$05110195 \%$ ALPHA TOTAL $\mu \mathrm{Ci} / \mathrm{mL}=$ ALPHA TOTAL $\mu \mathrm{Ci} / \mathrm{L} / 1000 \mathrm{~mL} /$

Relative Counting Error = [ |(The Square Root of TC + BKG * CT) / (TC - BKG *CT)| ] $1.96 * 100$

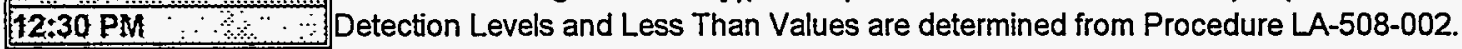

v RESULTS V

\begin{tabular}{|lcc|c|}
\hline ALPHA TOTAL in $\mu \mathrm{Ci} / \mathrm{mL} \quad$ (Average) $=$ & $2.72 E-05$ & DETECTION \\
& & \\
LEVEL & $1.10 \mathrm{E}-07$ \\
& & & $\mu \mathrm{Ci} / \mathrm{mL}$ \\
\hline
\end{tabular}

\begin{tabular}{|lll|}
\hline Data Entry by: & Date: & $05 / 11 / 95$ \\
\hline Approved by: & Date: & $5 / 11 / 55$ \\
\hline Form 508101_C Rev.1.3 & Page 1 of 1 & 09 \\
\hline
\end{tabular}




\section{AT : LA-508-101 (D-2)}

\section{LA-548-101 (A-3) SOLIDS}

\begin{tabular}{|c|c|c|c|}
\hline Whye & DETECTOR NUMBER & : 14 & 14 \\
\hline BLANK & 1,2, or 5 & अा\% & 2 \\
\hline $660 \mathrm{mom}$ & TOTAL COUNTS & अध 3 & ?3 \\
\hline 1367 & COUNT TIME in MINUTES & \% & 30 \\
\hline AT:OIF & BACKGROUND in cpm & $\because n_{n}$ & 0.3 \\
\hline AT & SAMPLE SIZE in $\mathrm{mL}$ & $\therefore 0.050$ & 0.050 \\
\hline \%USACOOE & DILUTION FACTOR & $\cdots$ & \\
\hline @ALPHA01 & DIGEST GRAMS of SOLIDS/L & 2.0712 & 2.0712 \\
\hline 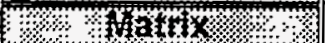 & EFFICIENCY FACTOR & 0.2274 & 0.2274 \\
\hline SOLID. & Lc, Rmax, or Rs,(SAMPLE RATE) & 0.233 & 0.233 \\
\hline SGapple & Sample Concentration in $\mu \mathrm{Ci} / \mathrm{g}$ & 4.46E-03 & BOOK\# \\
\hline S95T808: & Replicate Concentration in $\mu$ Ci/g & 4.46E-03 & 3.9 \\
\hline linstruiat & & & \\
\hline WB27807. & \multirow{7}{*}{\multicolumn{3}{|c|}{ 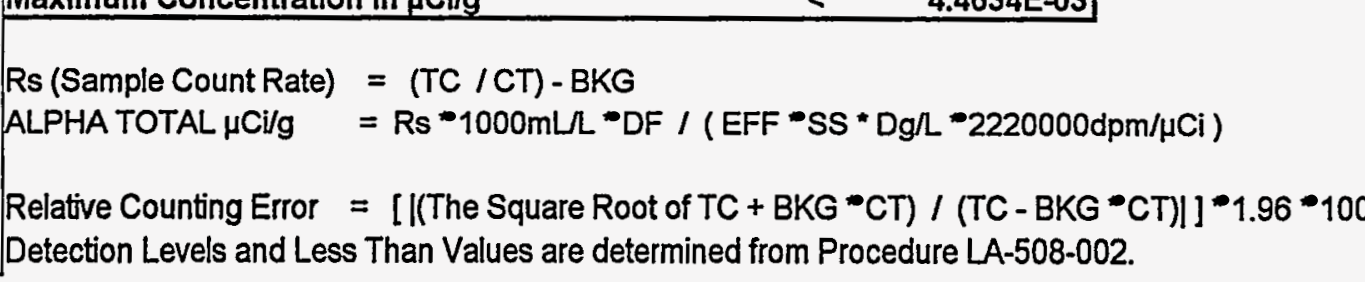 }} \\
\hline Ala & & & \\
\hline TLM & & & \\
\hline \%: & & & \\
\hline $5 / 10 / 95$ & & & \\
\hline H & & & \\
\hline & & & \\
\hline
\end{tabular}

$\checkmark$ RESULTS v

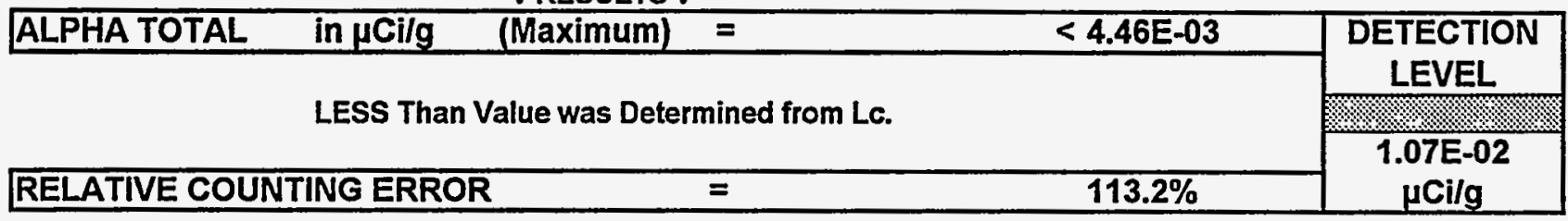

\begin{tabular}{|lll|}
\hline Data Entry by: X & Date: & $05 / 11 / 95$ \\
\hline Approved by: & Date: & $5 / 11 / 95$ \\
\hline Form 508101_C Rev. 1.3 & .70 & Page 1 of 1
\end{tabular}


AT : LA-508-101 (D-2)

\section{LA-548-101 (A-3) SOLIDS}

SAMPLE. REPLICATE

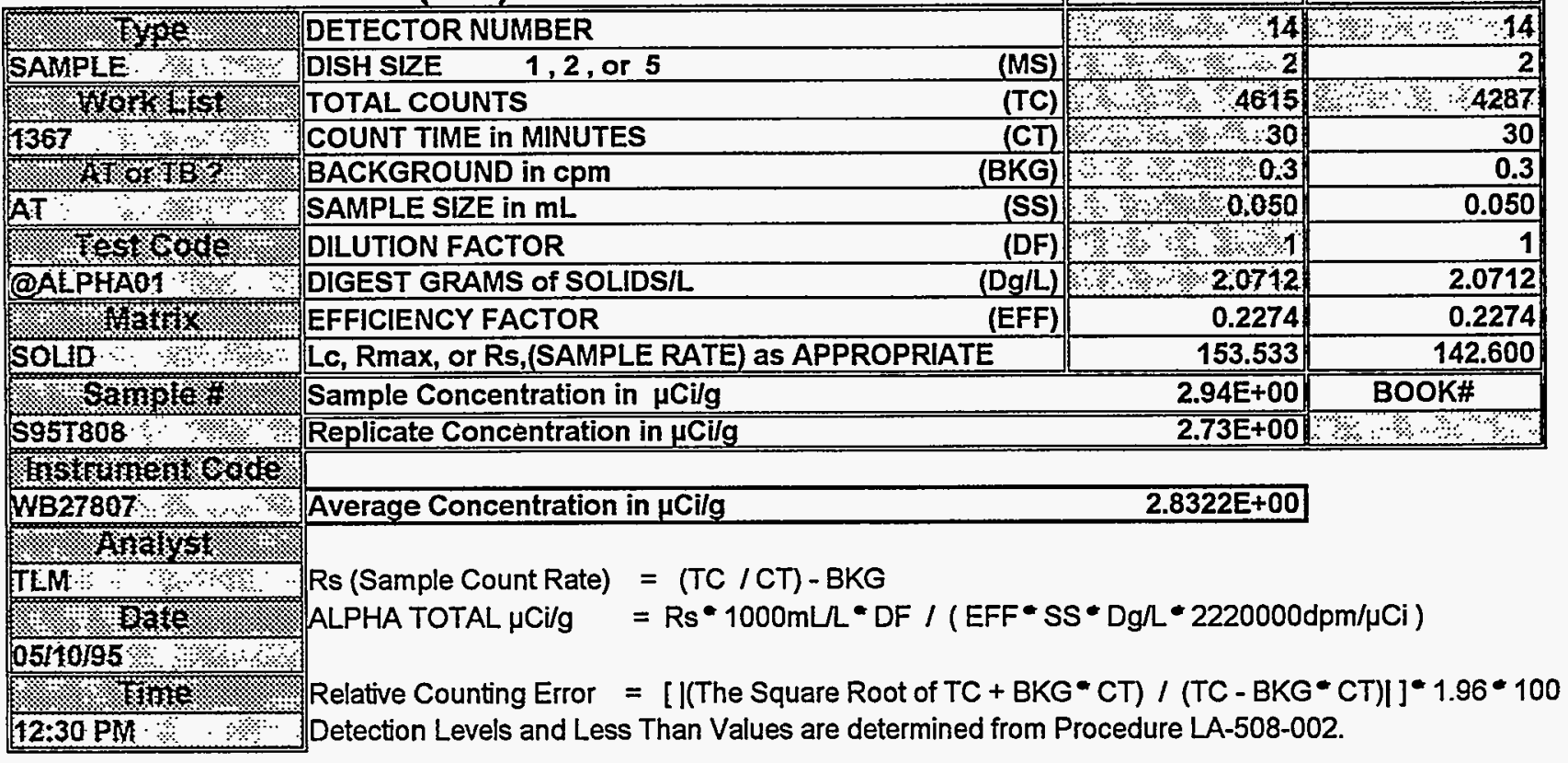

v RESULTS v

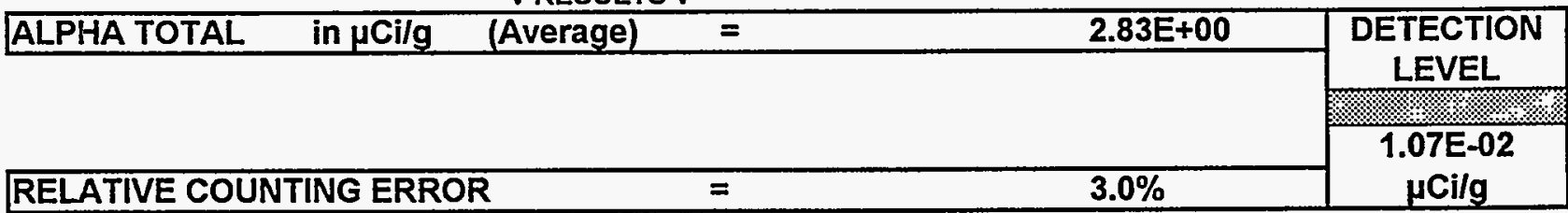

\begin{tabular}{|lll|}
\hline Data Entry by: & Date: & $05 / 11 / 95$ \\
\hline Approved by: & Date: & $5 / 11 / 95$ \\
\hline Form 508101_C Rev. 1.3 & 71 & Page 1 of 1 \\
\hline
\end{tabular}




\begin{tabular}{|c|c|c|c|}
\hline \multicolumn{2}{|c|}{ AT : LA-508-101 (D-2) } & \multirow[b]{2}{*}{ SAMPLE } & \multirow[b]{2}{*}{ REPLICATE } \\
\hline LA-548 & $8-101(A-3)$ & & \\
\hline ype & DETECTOR NUMBER & $3 \quad \square, 14$ & $6 \quad 6$ \\
\hline DUPLICATE : & DISH SIZE $\quad 1,2$, or 5 & 61: 2 & 2 \\
\hline$\% 110 \mathrm{~m} \% \mathrm{~K}$ & TOTAL COUNTS & 0.1777 & 1719 \\
\hline n? & COUNT TIME in MINUTES & $3 \longdiv { \square \square 3 0 }$ & 30 \\
\hline$\%$ \%o％ & BACKGROUND in cpm & थख 0.3 & 0.3 \\
\hline AT $\quad \therefore \quad \cdots$ & SAMPLE SIZE in $\mathrm{mL}$ & 0.050 & 0.050 \\
\hline Sestoocre & DILUTION FACTOR & $3 \quad 1$ & 1 \\
\hline @ALPHAO1 & DIGEST GRAMS of SOLIDSIL & $2 \quad 2.1844$ & 2.1844 \\
\hline 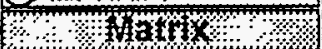 & EFFICIENCY FACTOR & 0.2274 & 0.2274 \\
\hline SOLID & Lc, Rmax, or Rs,(SAMPLE RATE) as APPROPRIATE & 58.933 & 57.000 \\
\hline S) Sampleq & Sample Concentration in $\mu \mathrm{Ci} / \mathrm{g}$ & $1.07 E+00$ & BOOK\# \\
\hline$\$ 95 T 808$ & Replicate Concentration in $\mu \mathrm{Ci} / \mathrm{g}$ & $1.03 \mathrm{E}+00$ & : \\
\hline 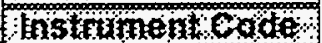 & & & \\
\hline WB27807: & Average Concentration in $\mu \mathrm{Ci} / \mathrm{g}$ & $1.0513 E+00$ & \\
\hline Wualyst & & & \\
\hline TKM $\quad \therefore \quad$ : & Rs (Sample Count Rate) $=(T C / C T)-B K G$ & & \\
\hline 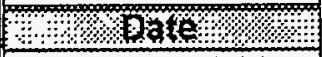 & ALPHA TOTAL $\mu \mathrm{Ci} / \mathrm{g}=\mathrm{Rs} * 1000 \mathrm{~mL} / \mathrm{D}^{*} \mathrm{DF} /(\mathrm{EFF} * \mathrm{~S}$ & $S * D g /$ * 2220000 & $\mathrm{pm} / \mu \mathrm{Ci}$ ) \\
\hline $05 / 10 / 95$ & & & \\
\hline 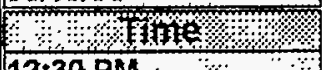 & ting Error $=[$ l $($ The Squ & & $\|] * 1$ \\
\hline & & & \\
\hline
\end{tabular}

V RESULTS V

\begin{tabular}{|llc|c|}
\hline ALPHA TOTAL in $\mu \mathrm{Ci} / \mathrm{g}$ (Average) $=$ & $1.05 E+00$ & $\begin{array}{c}\text { DETECTION } \\
\text { LEVEL }\end{array}$ \\
\hline RELATIVE COUNTING ERROR & $=$ & & $1.01 \mathrm{E}-02$ \\
$\mu \mathrm{Ci} / \mathrm{g}$
\end{tabular}

\begin{tabular}{|lll|}
\hline Data Entry by: Shyy & Date: & $05 / 11 / 95$ \\
\hline Approved by: & Date: & $5 / 11 / 95$ \\
\hline Form 508101_C Rev. 1.3 & 72 & Page 1 of 1 \\
\hline
\end{tabular}


PLACE ANALYTICAL CARD IN BOX BELOW OR ATTACH TRAVELER WHC-SD-WM-DP-112, REV. 0

AT : LA-508-101 (D-2)

\section{LA-548-101 (A-3) SOLIDS}

DETECTOR NUMBER

SAMPEE $\% \cdots \%$ DISH SIZE 1,2, or 5

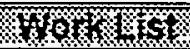

TOTAL COUNTS

1367: $: 3 . \%$ COUNT TIME in MINUTES

BACKGROUND in cpm

AT: $\because \%$ SAMPLE SIZE in $\mathrm{mL}$

IESCOCO DILUTION FACTOR

@AE.PHAO1

.

DIGEST GRAMS OF SOLIDS/L

SOLID: $\%$ Lc, Rmax, or Rs,(SAMPLE RATE) as APPROPRIATE

30

Sample Concentration in $\mu \mathrm{Ci} / \mathrm{g}$

$5957812 \%$ Replicate Concentration in $\mu \mathrm{Ci} / \mathrm{g}$

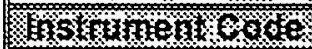

WB27807: $\quad$ Average Concentration in $\mu \mathrm{Ci} / \mathrm{g}$

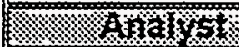

TLM $\%$ \% $\%$

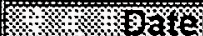

$05 ! 10195$

\%elative Counting Error = [ |(The Square Root of TC + BKG * CT) / (TC - BKG* CT)|] $1.96 * 100$

12:30.PM Detection Levels and Less Than Values are determined from Procedure LA-508-002.

v RESULTS v

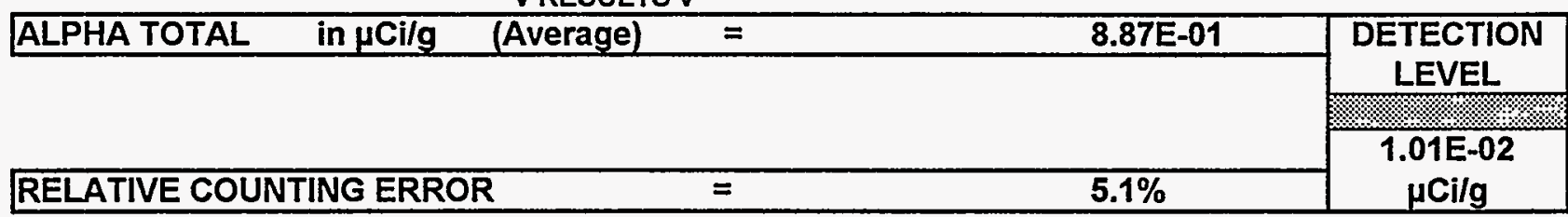

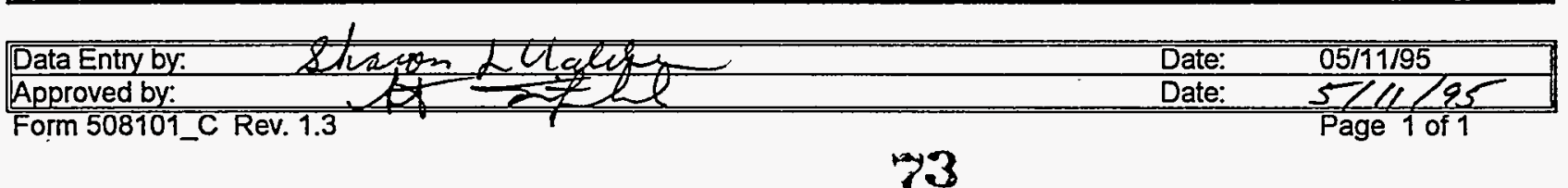




\begin{tabular}{|c|c|c|c|}
\hline \multicolumn{2}{|c|}{ AT : LA-508-101 (D-2) } & \multirow[b]{2}{*}{ SAMPLE } & \multirow[b]{2}{*}{ REPLICATE } \\
\hline LA-54 & $8-101(A-3)$ & & \\
\hline mof $y$ & DETECTOR NUMBER & $\because \because 8 \quad 14$ & 3 \\
\hline DUPLICATE & DISH SIZE $\quad 1,2$, or 5 & 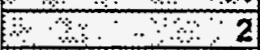 & 2 \\
\hline 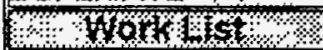 & TOTAL COUNTS & ए० 4411 & 4480 \\
\hline $1367: \therefore \because$ & COUNT TIME in MINUTES & $3 \times 30$ & 30 \\
\hline 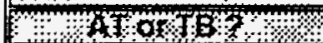 & BACKGROUND in cpm & $\begin{array}{l:lll} & 0.3 \\
\end{array}$ & 0.3 \\
\hline AT $\therefore$ & SAMPLE SIZE in $\mathrm{mL}$ & $\% \bigcirc \cdot 0.050$ & 0.050 \\
\hline ofestoode & DILUTION FACTOR & ४ ४ & 1 \\
\hline QALPHA01: & DIGEST GRAMS of SOLIDS/L & 2.21652 & 2.1652 \\
\hline otatox & EFFICIENCY FACTOR & 0.2274 & 0.2274 \\
\hline SOLID & Lc, Rmax, or Rs,(SAMPLE RATE) as APPROPRIATE & 146.733 & 149.033 \\
\hline $1:$ Samplox & Sample Concentration in $\mu \mathrm{Cilg}$ & $2.68 E+00$ & $\overline{B O O K \#}$ \\
\hline $595 T 812$ & Replicate Concentration in $\mu \mathrm{Ci} / \mathrm{g}$ & $2.73 \mathrm{E}+00$ & $3:$ \\
\hline Thstrumenuseader & & & \\
\hline WB27807 औ & Average Concentration in $\mu \mathrm{Ci} / \mathrm{g}$ & $2.7059 E+00$ & \\
\hline A: Andul & & & \\
\hline & Rs (Sample Count Rate) $=(\mathrm{TC} / \mathrm{CT})-\mathrm{BKG}$ & & \\
\hline OOAfe & $=R s^{*} 1000 \mathrm{~mL} L^{*} \mathrm{DF} /(\mathrm{EFF} * \mathrm{~S}$ & $S * D g / * 2220000$ & $\mathrm{pm} / \mu \mathrm{Ci})$ \\
\hline $05 / 10 / 95 \mathrm{3}$ & & & \\
\hline$\frac{1}{12 \cdot 30 \mathrm{PM}}$ & Relative Counting Error = & ( T & CT) $\mid] * 1.96$ * \\
\hline $12: 30 \mathrm{PM}$ & & & \\
\hline
\end{tabular}

$\checkmark$ RESULTS $v$

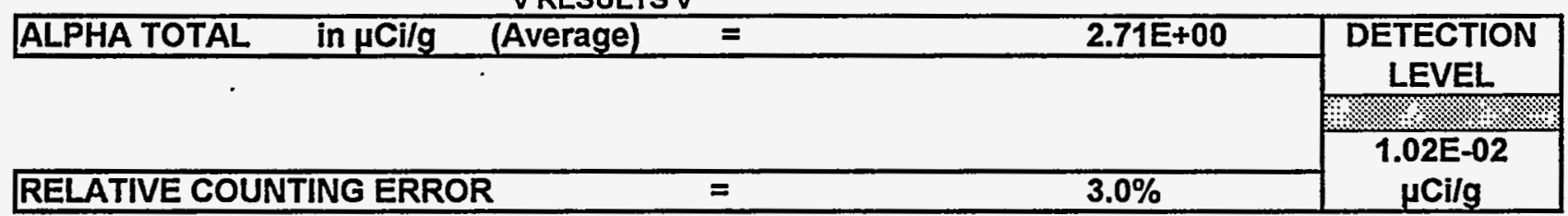

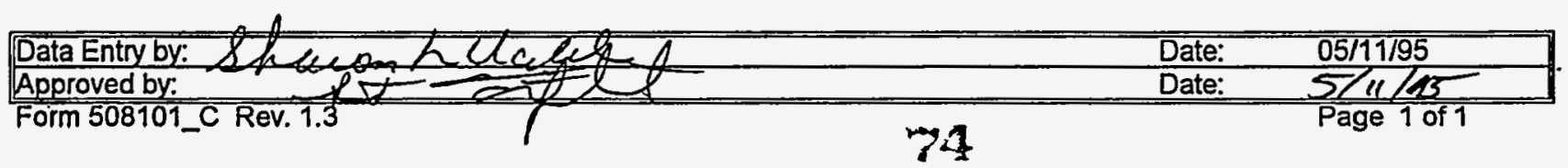




\section{LABCORE Data Entry Template for Worklist\# 1402}

\section{Analyst:}

\section{Instrument: $\mathrm{ABO0}$}

\section{Book \#}

Method: LA-508-101 Rev/Mod $1318 \stackrel{52 F}{5 / 15 / 95}$

Worldlist Comment: Re-entry of original data (Worklist 1366). SLF

\begin{tabular}{|c|c|c|c|c|c|c|c|c|c|c|}
\hline \multirow[t]{6}{*}{ GROUP } & PROJECT & S TYPE & SAMPLE\# & RA & -...-TEST $\cdots$ & MATRIX & ACTUAL & FOUND & DL & UNIT \\
\hline & & 1 STD & & & AALPHA01 ALPHAO1 & SOLID & & & $\mathrm{N} / \mathrm{A}$ & $u C i / g$ \\
\hline & & 1 STD & & & DALPHA01 ALPHA01E & SOLID & & & $N / A$ & \% ct. Error \\
\hline & & 2 BLNK-PREP & & & DALPHA01 ALPHA01 & SOLID & & & N/A & $\mathrm{uCi} / \mathrm{g}$ \\
\hline & & 2 BLNK-PREP & & & DALPHA01 ALPHAO1E & SOLID & & & $N / A$ & \% ct. Error \\
\hline & & 3 BLNK/BKG & & & DALPHA01 ALPHA01 & SOLID & & & N/A & $u C i / g$ \\
\hline 95000056 & $c-203$ & 4 SAMPLE & s95T000800 & $O F$ & DALPHAOI ALPHAO1 & SOLID & $N / A$ & & & $\mathrm{uCi} / \mathrm{g}$ \\
\hline 95000056 & $c-203$ & 4 SAMPLE & $\$ 95 T 000800$ & $0 \mathrm{~F}$ & DALPHAOI ALPHA01E & SOLID & N/A & & . & \% ct. Error \\
\hline 95000056 & $c-203$ & 5 DUP & $595 T 000800$ & $O F$ & DALPHA01 ALPHA01 & SOLID & & & N/A & $u C i / g$ \\
\hline 95000056 & $c-203$ & 5 DUP & S95T000800 & $O F$ & DALPHA01 ALPHA01E & SOLID & & & $\mathrm{H} / \mathrm{A}$ & \% ct. Error \\
\hline 95000056 & $c-203$ & 6 SPK & S95T000800 & $0 \mathrm{~F}$ & DALPHA01 ALPHA01 & SOLID & & & $N / A$ & $u C i / g$ \\
\hline 95000056 & $c-203$ & 7 SAMPLE & $595 T 000804$ & $O F$ & DALPHA01 ALPHA01 & SOLID & N/A & & & $u C i / g$ \\
\hline 95000056 & $\mathrm{C}-203$ & 7 SAMPLE & S95T000804 & $0 F$ & QALPHA01 ALPHA01E & SOLID & N/A & & & \% ct. Error \\
\hline 95000056 & $c-203$ & 8 DUP & $595 T 000804$ & $O F$ & QALPHA01 ALPHA01 & SOLID & & & N/A & $u C i / g$ \\
\hline 95000056 & $C-203$ & 8 DUP & S95T000804 & $O F$ & DALPHA01 ALPHA01E & SOLID & & & $N / A$ & \% ct. Error \\
\hline
\end{tabular}

\section{Final page for worklist \# 1402}

Analyst Signature

Date
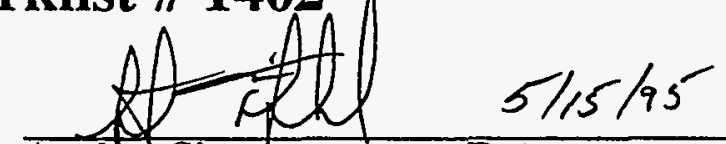

Analyst Signature

Date

Data Entry Comments:

Re-etry of Data

Wh 1318 (see

attached).

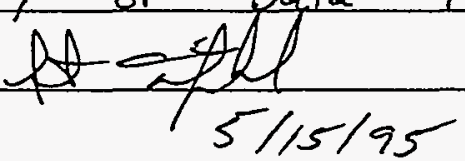

Units shown for QC (SPK \& STD) may not reflect the actual units. $D L=$ Detection Limit, $S=$ Worklist Slot Number,

$R=$ Replicate Number, $A=$ Aliquot Code. 


\section{WHC-SD-WM-DP-112, REV. 0}

THE FOLLOWING ANALYSES ARE INCLUDED IN THE DATA PACKAGE BUT THE RESULTS HAVE NOT BEEN REPORTED IN THE FINAL SUMMARY REPORTS. 


\section{Analyst:

$$
\text { SítH }
$$ \\ Instrument:}

Method: LA-508-101 Rev/Mod

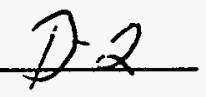

Worklist Comment: If dose rate warrants, run under RWP 273. SLF

\begin{tabular}{|c|c|c|c|c|c|c|c|c|c|c|}
\hline \multirow[t]{6}{*}{ GROUP } & PROJECT & S TYPE & SAHPLE\# & R A & - & MATRIX & ACTUAL & FOUND & DL & UNIT \\
\hline & & 1 STD & & & DALPHA01 ALPHA01 & SOLID & & & $\mathrm{H} / \mathrm{A}$ & $\mathrm{uCi} / \mathrm{g}$ \\
\hline & & 1 sto & & & DALPHA01 ALPHA01E & SOLID & & & N/A & * ct. Error \\
\hline & & 2 BLNK-PREP & & & DALPHA01 ALPHA01 & SOLID & & & $N / A$ & $\mathrm{uCi} / \mathrm{g}$ \\
\hline & & 2 BLNK-PREP & & & DALPHA01 ALPHA01E & SOLID & & & $\mathrm{N} / \mathrm{A}$ & $\%$ ct. Error \\
\hline & & 3 BLNK/BKG & - & & DALPHA01 ALPHA01 & SOLID & & & $N / A$ & $\mathrm{UCi} / \mathrm{g}$ \\
\hline 95000056 & $c-203$ & 4 SAMPLE & s95T000808 & OF & DALPHA01 ALPHA01 & SOLID & N/A & & & $\mathrm{uCi} / \mathrm{g}$ \\
\hline 95000056 & $c-203$ & 4 SAMPLE & s95T000808 & $0 \mathrm{~F}$ & DALPHA01 ALPHAO1E & SOLID & N/A & & & \% ct. Error \\
\hline 95000056 & $C-203$ & 5 DUP & $595 T 000808$ & $O F$ & DALPHA01 ALPHA01 & SOLID & & & $H / A$ & $\mathrm{uCi} / \mathrm{g}$ \\
\hline 95000056 & $C-203$ & 5 DUP & S95T000808 & $O F$ & DALPHA01 ALPHA01E & SOLID & & & $N / A$ & \% Ct. Error \\
\hline 95000056 & $C-203$ & 6 SAMPLE & s95T000812 & $O F$ & DALPHA01 ALPHA01 & SOLID & N/A & & & $u C i / g$ \\
\hline 95000056 & $c-203$ & 6 SAMPLE & S95T000812 & OF & DALPHA01 ALPHA01E & SOLID & N/A & & & \% Ct. Error \\
\hline 95000056 & $c-203$ & 7 DUP & s95T000812 & $O F$ & DALPHA01 ALPHA01 & SOLID & & & $N / A$ & $-\mathrm{uCi} / \mathrm{g}$ \\
\hline 95000056 & $c-203$ & 7 DUP & $s 957000812$ & $0 \mathrm{~F}$ & DALPHA01 ALPHA01E & SOLID & & & $N / A$ & - \% ct. Error \\
\hline
\end{tabular}

Final page for worklist \# 1287
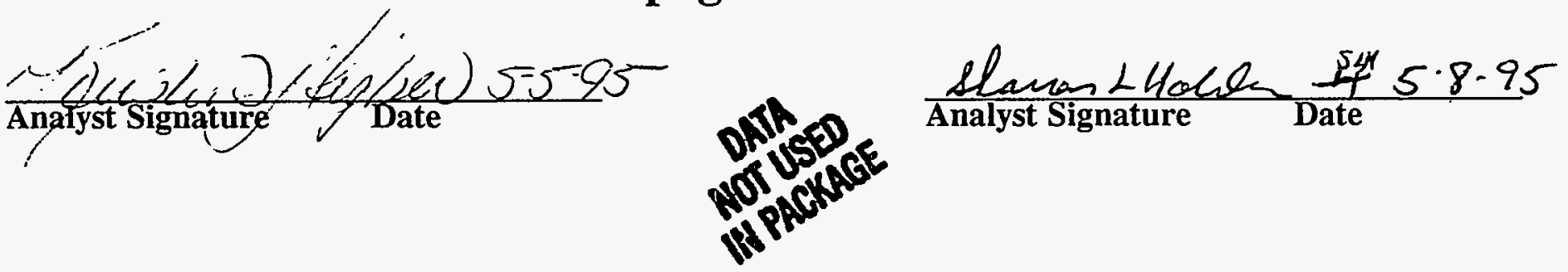

Data Entry Comments:

\section{RPD's for smples are out of ceath/}

Units shown for $Q C$ (SPK \& STD) may not reflect the actual units. $D L=$ Detection Limit, $S=$ Worklist Slot Number, $R=$ Replicate Number, $A=$ Aliquot Code. 


\begin{tabular}{|c|c|c|c|}
\hline \multicolumn{4}{|c|}{ AT : LA-508-101 (D-2) } \\
\hline \multicolumn{2}{|c|}{ LA-548-101 (A-3) } & STANDARD & REPLICATE \\
\hline Wyper & DETECTOR NUMBER & $1 \%$ २ 16 & \%४ 16 \\
\hline STANDARD: & DISH SIZE $\quad 1,2$, or 5 & $2 \quad 2$ & 2 \\
\hline BWor & TOTAL COUNTS & .4443 & 4416 \\
\hline 1287. & COUNT TIME in MINUTES & $81 \quad \because 30$ & 30 \\
\hline \%. AT ox B B & BACKGROUND in cpm & $\therefore 3$ & 0.6 \\
\hline 3 & SAMPLE SIZE in $\mathrm{mL}$ & $8 \quad 2010.000$ & 10.000 \\
\hline Testode & DILUTION FACTOR & \% $\because 1$ & 1 \\
\hline QAEPHA01: & DIGEST DILUTION FACTOR & ह. & 1 \\
\hline 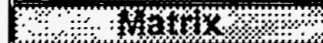 & EFFICIENCY FACTOR & 0.2104 & 0.2104 \\
\hline LIQUID & LC, Rmax, or Rs, (SAMPLE RATE) as APPROPRIATE & 147.500 & 136.600 \\
\hline Sample: & Sample Concentration in $\mu \mathrm{Ci} / \mathrm{L}$ & $3.16 \mathrm{E}-02$ & BOOK\# \\
\hline WRKLSTH 287. & Replicate concentration in $\mu \mathrm{Ci} / \mathrm{L}$ & $2.92 \mathrm{E}-02$ & 107B52: \\
\hline Fotriment 60 othe & & & \\
\hline WB27806 & Average Concentration in $\mu \mathrm{Ci} / \mathrm{L}$ & $3.0412 E-02$ & \\
\hline Andusto & & & \\
\hline KGH & Rs (Sample Count Rate) & & \\
\hline $05 / 05 / 95$ & $\begin{array}{ll}\text { ALPHA TOTAL } \mu \mathrm{Ci} / \mathrm{R} & =\mathrm{Rs} * 1000 \mathrm{mLL} * \mathrm{DF} * \mathrm{DDF} /(\mathrm{EF} \\
\text { ALPHA TOTAL } \mu \mathrm{Ci} / \mathrm{mL} & =\text { ALPHA TOTAL } \mu \mathrm{Ci} \Omega / 1000 \mathrm{~mL}\end{array}$ & $S S * 2220000$ & $\mu \mathrm{Ci})$ \\
\hline 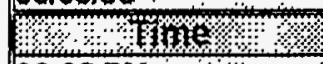 & Relative Counting Error $=[\mid($ The & $(\mathrm{TC}$ & $\bullet C T)[] \cdot 1.96 \bullet 1$ \\
\hline $2: 00 \mathrm{PM}$ & Is and Less & & \\
\hline
\end{tabular}

v RESULTS V

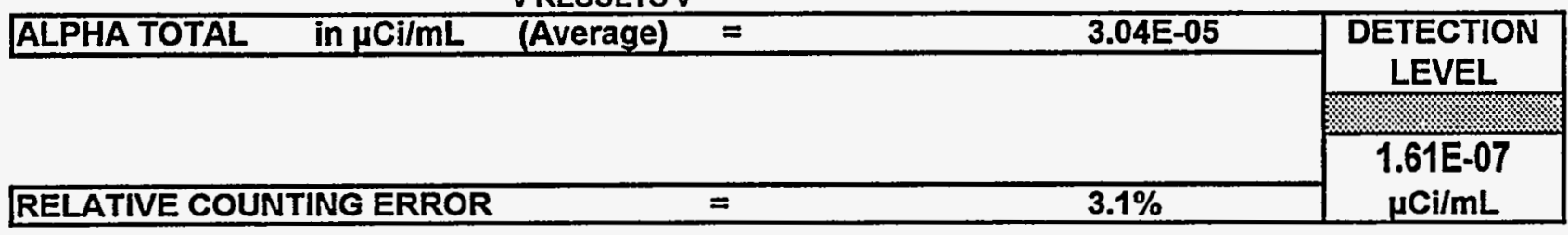

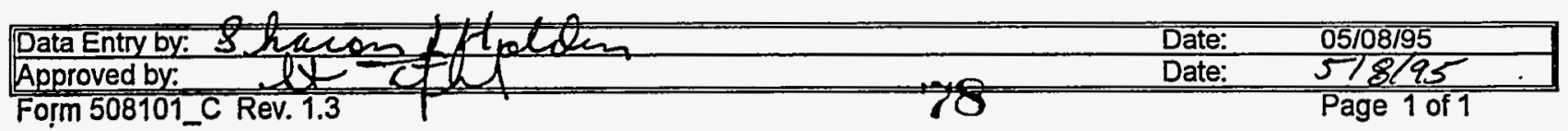


PLACE ANALYTICAL CARD IN BOX BELOW OR ATTACH TRAVELER WHC-SD-WM-DP-112, REV 0

\begin{tabular}{|c|c|c|c|}
\hline \multicolumn{4}{|c|}{ AT : LA-508-101 (D-2) } \\
\hline \multicolumn{2}{|c|}{ LA-548-101 (A-3) } & BLANK & REPLICATE \\
\hline tos & DETECTOR NUMBER & $\$ ? . .16$ & $. \% \div \div 16$ \\
\hline BEANK $\%$ & DISH SIZE & 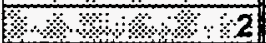 & 2 \\
\hline 1. & TOTAL COUNTS & \% : .811 & ०००:2 \\
\hline $1287 \%$ & COUNT TIME in MINUTES & \% & 30 \\
\hline X & BACKGROUND in cpm & $\% \% \because 0.6$ & 0.6 \\
\hline AT $: \cdots$ & SAMPLE SIZE in $\mathrm{mL}$ & $\$ \%, 0.050$ & 0.050 \\
\hline 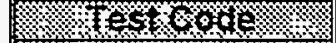 & DILUTION FACTOR & \%: $: 4$ & 1 \\
\hline @AEPHAOI & DIGEST GRAMS of SOLIDS/L & $1 \%, 2 \% 20712$ & 2.0712 \\
\hline (1) & EFFICIENCY FACTOR & 0.2104 & 0.2104 \\
\hline SOLID $\because 2, \%$ & LC, Rmax, or Rs,(SAMPLE RATE) as APPROPRIATE & 0.330 & 0.330 \\
\hline 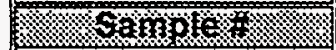 & Sample Concentration in $\mu \mathrm{Ci} / \mathrm{g}$ & $6.82 E-03$ & BOOK\# \\
\hline $595 T 808: \%$ & Replicate Concentration in $\mu \mathrm{Ci} / \mathrm{g}$ & 6.82E-03 & \% \\
\hline 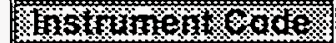 & & & \\
\hline WB27806: & Maximum Concentration in $\mu \mathrm{Ci} / \mathrm{g}$ & 6.8222E-03 & \\
\hline 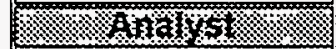 & & & \\
\hline KGH : & Rs (Sample Count Rate) $=(T C / C T)-B K G$ & & \\
\hline 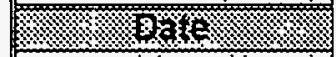 & $=R s * 1000 \mathrm{~mL} / \mathrm{DF}^{*} /(\mathrm{EFF} * \mathrm{~s}$ & $S * D g / L * 2220000 c$ & $\mathrm{pm} / \mu \mathrm{Ci})$ \\
\hline $05105195 \%, \%$ & & & \\
\hline $02: 00 \mathrm{PM}$ & $\begin{array}{l}\text { Relative Counting Error }=[\text { [ }(\text { The Square Root of TC }+\mathrm{BK} \\
\text { Detection Levels and Less Than Values are determined from }\end{array}$ & $\begin{array}{l}\text { CT) / (TC - BK } \\
\text { ocedure LA-508- }\end{array}$ & $* C T) \mid] * 1.96 * 100$ \\
\hline
\end{tabular}

v RESULTS V

\begin{tabular}{|lcc|c|}
\hline ALPHA TOTAL in $\mu \mathrm{Ci} / \mathrm{g} \quad($ Maximum) $=$ & $<6.82 E-03$ & $\begin{array}{c}\text { DETECTION } \\
\text { LEVEL }\end{array}$ \\
LESS Than Value was Determined from Lc. & & \\
\hline RELATIVE COUNTING ERROR & $=$ & $150.8 \%$ & $1.55 \mathrm{E}-02$ \\
$\mu \mathrm{Ci} / \mathrm{g}$ & \\
\hline
\end{tabular}

\begin{tabular}{|lll|}
\hline Data Entry by: & Date: & $05 / 08 / 95$ \\
\hline Approved by. & Date: & $3 / 8 / 55$ \\
\hline Form 508101_C Rev. 1.3 & 79 & Page 1 of 1
\end{tabular}


PLACE ANALYTICAL CARD IN BOX BELOW OR ATTACH TRAVELER WHC-SD-WM-DP-112, REV. 0

\begin{tabular}{|c|c|c|c|}
\hline \multicolumn{2}{|c|}{ AT : LA-508-101 (D-2) } & \multirow{3}{*}{$\frac{\text { SAMPLE }}{16}$} & \multirow[b]{2}{*}{ REPLICATE } \\
\hline \multicolumn{2}{|c|}{ LA-548-101 (A-3) } & & \\
\hline$\%$ १ype & DETECTOR NUMBER & & $16)$ \\
\hline SAMPLE $\because$ & DISH SIZE & $\% .82$ & 2 \\
\hline \%OKOS & TOTAL COUNTS & +ै. 44815 & $2+4620$ \\
\hline $1287 \div \because \cdots$ & COUNT TIME in MINUTES & 3.30 & 30 \\
\hline HAT 0\% B\% & BACKGROUND in cpm & $\therefore<\quad 0.6$ & 0.6 \\
\hline AT & SAMPLE SIZE in $\mathrm{mL}$ & $8 \% 0.050$ & 0.050 \\
\hline OTSKoode & DILUTION FACTOR & 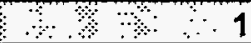 & 1 \\
\hline QALPHAOT & DIGEST GRAMS of SOLIDS/L & $2: 2.0712$ & 2.0712 \\
\hline matro & EFFICIENCY FACTOR & 0.2104 & 0.2104 \\
\hline SOLID: & Lc, Rmax, or Rs,(SAMPLE RATE) as APPROPRIATE & 159.900 & 153.400 \\
\hline S Gampla: & Sample Concentration in $\mu \mathrm{Ci} / \mathrm{g}$ & $3.31 \mathrm{E}+00$ & BOOK\# \\
\hline $595 T 808 \cdots$ & Replicate Concentration in $\mu \mathrm{Ci} / g$ & $3.17 E+00$ & $\because \because$ \\
\hline Frstariment $C$ odde & & & \\
\hline WB27806 \&..? & Average Concentration in $\mu \mathrm{Ci} / g$ & $3.2385 \mathrm{E}+00$ & \\
\hline \%.Antaly & & & \\
\hline KGH $\ldots \cdots$ & Rs (Sample Count Rate) $=(\mathrm{TC} / \mathrm{CT})-\mathrm{BKG}$ & & \\
\hline$\%$ & $=\mathrm{Rs}^{\bullet} 1000 \mathrm{~mL} / \bullet \mathrm{DF} /(\mathrm{EFF} \bullet \mathrm{s}$ & $s \cdot D g / L \cdot 2220000$ & $\mathrm{pm} / \mu \mathrm{Ci})$ \\
\hline $05105195 \ldots$ & & & \\
\hline Con & [ I (The Square Root of TC $+\mathrm{BH}$ & ITC & CT) $\mid=1.96 * 100$ \\
\hline 65.00 PM: & & & \\
\hline
\end{tabular}

V RESULTS V

\begin{tabular}{|lll|c|}
\hline ALPHA TOTAL in $\mu \mathrm{Ci} / \mathrm{g}$ (Average) & $=$ & $3.24 \mathrm{E}+00$ & $\begin{array}{c}\text { DETECTION } \\
\text { LEVEL }\end{array}$ \\
& & & \\
\hline RELATIVE COUNTING ERROR & $=$ & $2.9 \%$ & $1.55 \mathrm{E}-02$ \\
$\mu \mathrm{Ci} / \mathrm{g}$ & \\
\hline
\end{tabular}

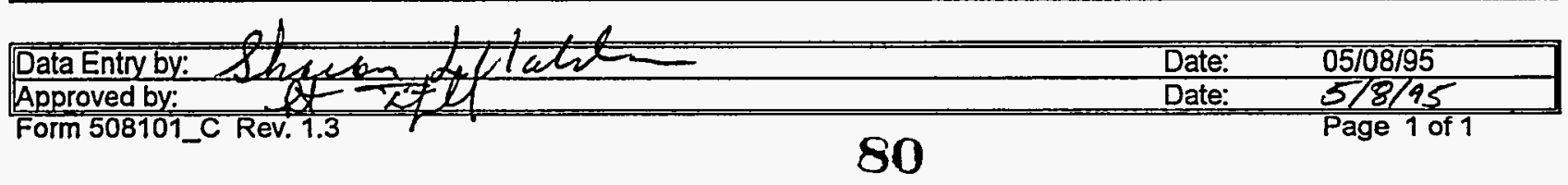




\section{AT : LA-508-101 (D-2)}

\section{LA-548-101 (A-3) SOLIDS}

\begin{tabular}{|c|c|c|}
\hline Kov & DETECTOR NUMBER & $4 \%$ \\
\hline DUPEICAEE & DISH SIZE & \% \\
\hline 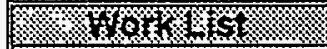 & TOTAL COUNTS & 9.9786 \\
\hline 1287.0 .6 & COUNT TIME in MINUTES & \%०:०. 30 \\
\hline 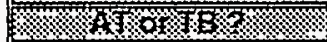 & BACKGROUND in cpm & $\% \% \%: 0.6$ \\
\hline \% & SAMPLE SIZE in $\mathrm{mL}$ & $\%, \%, 0,050$ \\
\hline 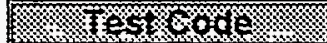 & DILUTION FACTOR & ४०००\%1 \\
\hline @AEPHAOT ? & DIGEST GRAMS of SOLIDS/L & . $2,2,1844$ \\
\hline$\because$ fatfo & EFFICIENCY FACTOR & 0.2104 \\
\hline SOLIQ $\%$ & LC, Rmax, or Rs,(SAMPLE RATE) as APPROPRIATE & 58.933 \\
\hline 8 Samplo & Sample Concentration in $\mu \mathrm{Ci} / \mathrm{g}$ & $1.16 \mathrm{E}+00$ \\
\hline $5957808 \% ?$ & Replicate Concentration in $\mu \mathrm{Ci} / \mathrm{g}$ & $1.23 \mathrm{E}+00 \mathrm{O} / \%$ \\
\hline 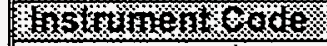 & & \\
\hline WB27806\% \% & Average Concentration in $\mu \mathrm{Ci} / \mathrm{g}$ & $1.1911 E+00$ \\
\hline 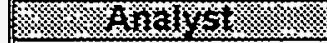 & & \\
\hline $\mathrm{KGH}: \%$ & \multirow{4}{*}{\multicolumn{2}{|c|}{$\begin{array}{l}\text { Rs (Sample Count Rate) }=(\mathrm{TC} / \mathrm{CT})-\mathrm{BKG} \\
\text { ALPHA TOTAL } \mu \mathrm{Ci} / \mathrm{g}=\mathrm{Rs} \bullet 1000 \mathrm{~mL} / \cdot \mathrm{DF} /(\mathrm{EFF} \bullet \mathrm{SS} \bullet \mathrm{Dg} / \mathrm{L} \cdot 2220000 \mathrm{dpm} / \mu \mathrm{Ci}) \\
\text { Relative Counting Error }=\left[\left((T h e \text { Square Root of TC }+\mathrm{BKG} \bullet \mathrm{CT}) /\left(\mathrm{TC}-\mathrm{BKG}{ }^{*} \mathrm{CT}\right) \mid\right]^{\bullet} 1.96 \bullet 100\right. \\
\text { Detection Levels and Less Than Values are determined from Procedure LA-508-002. }\end{array}$}} \\
\hline $05105195 \%$ & & \\
\hline 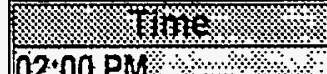 & & \\
\hline & & \\
\hline
\end{tabular}

V RESULTS $V$

\begin{tabular}{|llc|c|}
\hline ALPHA TOTAL in $\mu \mathrm{Ci} / \mathrm{g}$ (Average) $=$ & $1.19 \mathrm{E}+00$ & $\begin{array}{c}\text { DETECTION } \\
\text { LEVEL }\end{array}$ \\
\hline RELATIVE COUNTING ERROR & $=$ & & $1.47 \mathrm{E}-02$ \\
$\mu \mathrm{Ci} / \mathrm{g}$ & \\
\hline
\end{tabular}

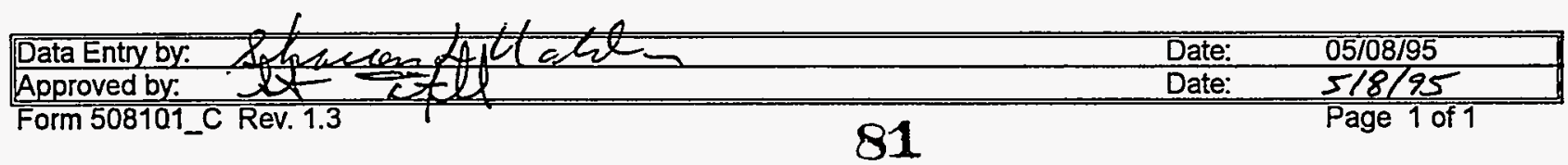




\begin{tabular}{|c|c|c|c|}
\hline & \multirow[b]{2}{*}{ SAMPLE } & \multirow[b]{2}{*}{ REPLICATE } \\
\hline \multicolumn{2}{|c|}{$\begin{array}{r}\text { AT: LA-508-101 (D-2) } \\
\text { LA-548-101 (A-3) }\end{array}$} & & \\
\hline Yets: & DETECTOR NUMBER & 30116 & +1! \\
\hline SAMPLE: & DISH SIZE & $\because \because 2$ & 2 \\
\hline WOXKLIS & TOTAL COUNTS & $3 \% 1566$ & 1529 \\
\hline 1287 & COUNT TIME in MINUTES & $\because \%$ & 30 \\
\hline \% & BACKGROUND in cpm & $\because \quad 0: 6$ & 0.6 \\
\hline AT: $\because$ & SAMPLE SIZE in $\mathrm{mL}$ & $0,0,0$ & 0.050 \\
\hline 205000 & DILUTION FACTOR & ख० $: 1$ & 1 \\
\hline QALPHAO1 & DIGEST GRAMS of SOLIDS/L & :. 2.19 & 2.19 \\
\hline 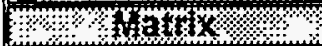 & EFFICIENCY FACTOR & 0.2104 & 0.2104 \\
\hline SOLID & LC, Rmax, or Rs,(SAMPLE RATE) as APPROPRIATE & 51.600 & 50.367 \\
\hline Samplo: & Sample Concentration in $\mu \mathrm{Cilg}$ & $1.01 E+00$ & BOOK\# \\
\hline S95T812. & Replicate Concentration in $\mu \mathrm{Ci} / \mathrm{g}$ & $9.85 \mathrm{E}-01$ & $\because \quad \because$ \\
\hline Insthumento o det & & & \\
\hline WB27806: * & Average Concentration in $\mu \mathrm{Ci} / \mathrm{g}$ & 9.9682E-01 & \\
\hline Amaly & & & \\
\hline KGH $: \cdots$ & Rs (Sample Count Rate) $=(\mathrm{TC} / \mathrm{CT}$ ) - BKG & & \\
\hline Woures\% & $=R s * 1000 \mathrm{~mL} / * D F /(E F F * s$ & $s * D g / \cdot 222000$ & $\mathrm{~m} / \mu \mathrm{Ci})$ \\
\hline 05105195 & & & \\
\hline$-1,3,10 \%$ & re Root of TC & (T & T) $]^{*}$ \\
\hline & & & \\
\hline
\end{tabular}

V RESULTS V

\begin{tabular}{|llc|c|}
\hline ALPHA TOTAL in $\mu \mathrm{Ci} / \mathrm{g}$ (Average) $=$ & $9.97 \mathrm{E}-01$ & $\begin{array}{c}\text { DETECTION } \\
\text { LEVEL }\end{array}$ \\
\hline RELATIVE COUNTING ERROR & $=$ & & $1.47 \mathrm{E}-02$ \\
& & $5 \mathrm{Ci} / \mathrm{g}$ \\
\hline
\end{tabular}

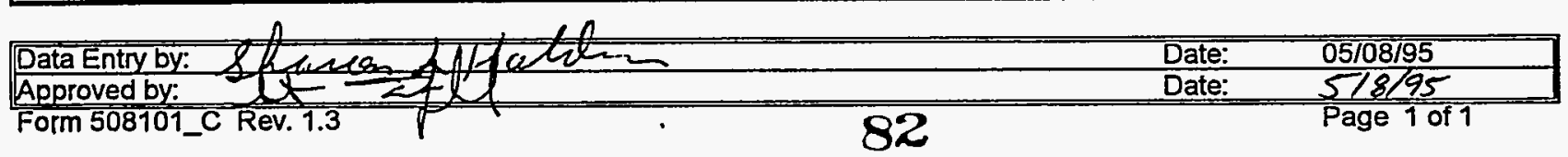


WHC-SD-WM-DP-172, Rev. 0

PLACE ANALYTICAL CARD IN BOX BELOW OR ATTACH TRAVELER

AT : LA-508-101 (D-2)

LA-548-101 (A-3)

SOLIDS

\begin{tabular}{|c|c|c|c|}
\hline F & DETECTOR NUMBER & $4 \% \div 16$ & $3 \% 16$ \\
\hline DUPEICATE $\because$ & DISH SIZE & \%०\% 2 & 2 \\
\hline 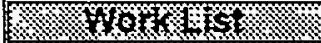 & TOTAL COUNTS & ४०\%4666 & \%०० 4395 \\
\hline $1287 . * \%$ & COUNT TIME in MINUTES & ६ै४२ ४ 30 & 30 \\
\hline \% $1 \% 60 \% 8 \%$ & BACKGROUND in cpm & $\% \% \%: 0.6$ & 0.6 \\
\hline AT $\cdots \cdots$ & SAMPLE SIZE in $\mathrm{mL}$ & $8 \%, \%, 0,050$ & 0.050 \\
\hline SES \&OOR & DILUTION FACTOR & अे $० 1$ & 1 \\
\hline @ALPHAOT: & DIGEST GRAMS of SOLIDSIL & 2.2 .21652 & 2.1652 \\
\hline 拱a & EFFICIENCY FACTOR & 0.2104 & 0.2104 \\
\hline SOLID : \% & LC, Rmax, or Rs,(SAMPLE RATE) as APPROPRIATE & 154.933 & 145.900 \\
\hline $\left.8 a n_{0}\right)$ & Sample Concentration in $\mu \mathrm{Ci} / \mathrm{g}$ & $3.06 \mathrm{E}+00$ & BOOK\# \\
\hline $5957812 \%$ & Replicate Concentration in $\mu \mathrm{Cilg}$ & $2.89 \mathrm{E}+00$ & ॥ै। \\
\hline
\end{tabular}

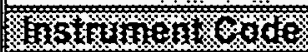

WB27806\% : $\because$ Average Concentration in $\mu \mathrm{Ci} / \mathrm{g}$

$2.9746 \mathrm{E}+00$

.

KGH: $:$ Rs (Sample Count Rate) $=($ TC / CT) - BKG

ALPHA TOTAL $\mu \mathrm{Ci} / \mathrm{g}=\mathrm{Rs}$ * 1000mLL * DF / ( EFF * SS * Dg/L * 2220000dpm/ $\mu \mathrm{Ci}$ )

$05105195: \cdots \%$

Felative Counting Error = [ (The Square Root of TC+BKG *CT) / (TC - BKG * CT)| ] * 1.96 * 100

$02: 00$ PM $: 3$ Detection Levels and Less Than Values are determined from Procedure LA-508-002.

V RESULTS V

\begin{tabular}{|llc|c|}
\hline ALPHA TOTAL in $\mu \mathrm{Ci} / \mathrm{g}$ (Average) $=$ & $2.97 \mathrm{E}+00$ & $\begin{array}{c}\text { DETECTION } \\
\text { LEVEL }\end{array}$ \\
\hline RELATIVE COUNTING ERROR & $=$ & & \\
$1.48 \mathrm{E}-02$ \\
$\mu \mathrm{Ci} / \mathrm{g}$
\end{tabular}

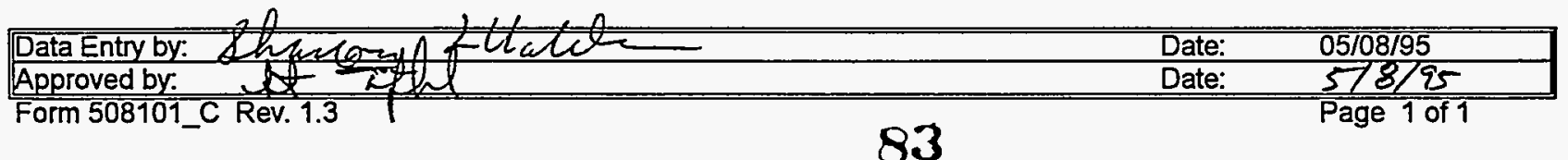

